\title{
CONVECTION IN SUPERPOSED LIQUID AND POROUS LAYERS WITH HEAT AND MASS TRANSFER AT THE FREE SURFACE
}

by

Rita Kozak, B.Eng.

Ryerson University, Toronto, 2003

\author{
A thesis \\ presented to Ryerson University \\ in partial fulfillment of the \\ requirements for the degree of \\ Master of Applied Science \\ in the program of \\ Mechanical Engineering
}

Toronto, Ontario, Canada

(C) Rita Kozak 2005 


\section{UMI Number: EC53036}

\section{All rights reserved \\ INFORMATION TO USERS}

The quality of this reproduction is dependent upon the quality of the copy submitted. Broken or indistinct print, colored or poor quality illustrations and photographs, print bleed-through, substandard margins, and improper alignment can adversely affect reproduction.

In the unlikely event that the author did not send a complete manuscript and there are missing pages, these will be noted. Also, if unauthorized copyright material had to be removed, a note will indicate the deletion.

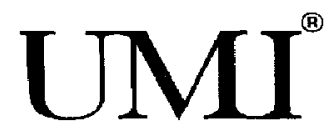

UMI Microform EC53036

Copyright 2008 by ProQuest LLC

All rights reserved. This microform edition is protected against unauthorized copying under Title 17, United States Code.

ProQuest LLC

789 East Eisenhower Parkway

P.O. Box 1346

Ann Arbor, MI 48106-1346 


\section{AUTHOR'S DECLARATION}

I herby declare that I am the sole author of this thesis.

I authorize Ryerson University to lend this thesis to other institutions or individuals for the purpose of scholarly research.

\section{,}

I further authorize Ryerson University to reproduce this thesis by photocopying or by other means, in total or in part, at the request of other institutions or individuals for the purpose of scholarly research.

$p_{n}$ 


\title{
ABSTRACT \\ CONVECTION IN SUPERPOSED LIQUID AND POROUS LAYERS WITH HEAT AND MASS TRANSFER AT THE FREE SURFACE
}

\author{
Rita Kozak \\ Master of Applied Science \\ in the program of \\ Mechanical Engineering, 2005 \\ School of Graduate Studies, Ryerson University, Toronto
}

In the present study, the onset of thermal convection in a liquid layer overlying a porous layer in the presence of a free surface with evaporation is investigated. The NavierStokes equations are solved for the liquid layer, while the Brinkman model describes the porous layer. Two and three-dimensional geometrical models are considered, with bottom heating and lateral heating. Buoyancy convection, thermocapillary convection and combined convection are studied in detail for different aspect ratios. For the evaporation analysis, two conditions are treated simultaneously at the free surface. In the first, surface tension is assumed to vary linearly with temperature. In the second, a linear saturation temperature is imposed at the free surface to allow evaporation. Different aspect ratios, thickness ratios as well as Marangoni numbers are studied in detail. Results reveal the significant effect evaporation in the liquid layer has on convective flow patterns. 


\section{ACKNOWLEDGEMENTS}

The author would like to sincerely thank Dr. Ziad Saghir of Ryerson University for his helpful assistance, guidance and encouragement throughout the completion of this thesis. The author would also like to express her appreciation to Artur Chaber and both her parents Bozena Spencer and Adam Kozak for their support. The author also acknowledges the support and useful suggestions of her colleagues Omar Gaber, Leily Abidi and Theo Makriyannis. 


\section{TABLE OF CONTENTS}

Author's Declaration $\quad$ ii

Abstract

Acknowledgements iv

Table of Contents $v \mathbf{v}$

List of Tables

List of Figures $\quad$ ix

Nomenclature $\quad$ xii

CHAPTER 1 - Introduction and Literature Review

1.1 Introduction 1

1.2 Onset of Convection in Liquid and Porous Cavity 2

$\begin{array}{lll}1.3 & \text { Evaporation in Liquid } & 8\end{array}$

$\begin{array}{lll}1.4 & \text { Research Objectives } & 10\end{array}$

CHAPTER 2 - Governing Equations and Numerical Solution 13

2.1 Model Description $\quad 13$

2.2 Liquid Layer Governing Equations $\quad 15$

2.2.1 Continuity Equation 15

2.2.2 Momentum Balance Equation 15

$\begin{array}{ll}\text { 2.2.3 Energy Balance Equation } & 16\end{array}$

2.3 Porous Layer Governing Equations 16

$\begin{array}{ll}\text { 2.3.1 Continuity Equation } & 17\end{array}$

$\begin{array}{ll}\text { 2.3.2 Momentum Balance Equation } & 17\end{array}$

$\begin{array}{lll}2.3 .3 & \text { Energy Balance Equation } & 18\end{array}$

2.4 Non-Dimensional Analysis 19

2.5 Non-Dimensional Liquid Layer Governing Equations 19 
2.5.1 Continuity Equation $\quad 19$

2.5.2 Momentum Balance Equation 19

2.5.3 Energy Balance Equation 20

2.6 Non-Dimensional Porous Layer Governing Equations 20

2.6.1 Continuity Equation 20

2.6.2 Momentum Balance Equation 20

2.6.3 Energy Balance Equation 21

2.7 Model Boundary Conditions 23

2.7.1 Bottom Heating Boundary Condition 23

2.7.2 Lateral Heating Boundary Condition 24

2.7.3 Evaporation Boundary Condition 25

2.8 Numerical Solution Technique 27

2.8.1 Finite Element Analysis 28

2.8.2 Mesh Sensitivity Analysis $\quad 29$

CHAPTER 3 - Superposed Liquid and Porous Layer with Adiabatic Free 33 Surface

3.1 Two-Dimensional Modeling 33

3.1.1 Bottom Heating Buoyancy Convection 33

3.1.2 Bottom Heating Marangoni Convection 37

3.1.3 Lateral Heating Buoyancy Convection 40

3.1.4 Lateral Heating Marangoni Convection 42

3.1.5 Lateral Heating Combined Buoyancy and Marangoni 47 Convection

3.2 Three-Dimensional Modeling 49

3.2.1 Lateral Heating $\quad 49$

3.2.2 Bottom Heating 57

CHAPTER 4 - Superposed Liquid and Porous Layer with Evaporation at 61 Free Surface 
4.1 Introduction 61

4.2 Lateral Heating $\quad 61$

CHAPTER 5 - Conclusion $\quad 69$

APPENDIX A - Non-Dimensional Analysis of Governing Equations

B.1 Bottom Heating 71

$\begin{array}{lll}\text { B.2 Lateral Heating } & 79\end{array}$

APPENDIX B - Input Files

C.1 Marangoni Convection Lateral Heating 83

C.2 Evaporation Lateral Heating 85

$\begin{array}{lr}\text { REFERENCES } & 89\end{array}$ 


\section{LIST OF TABLES}

Table 1 Calculated Nusselt numbers for mesh sensitivity 


\section{LIST OF FIGURES}

Figure 1

Figure 2

Figure 3

Figure 4

Figure 5

Figure 6

Figure 7

Figure 8

Figure 9

Figure 10

Figure 11

Figure 12

Figure 13

Figure 14

Figure 15

Figure 16
Geometrical model of cavity

Bottom heating boundary condition

Lateral heating boundary condition

Evaporation boundary condition

Node numbers for key-points

Finite element mesh for model defined as $\mathrm{X}$-axis by $\mathrm{Y}$-axis elements

Buoyancy convection in the cavity for different Biot numbers $(\mathrm{AR}=1, \mathrm{Pr}=7)$

Streamlines and temperature variation in the cavity for different aspect ratios $\left(\mathrm{Ra}_{\mathrm{L}}=100, \mathrm{Ra}_{\mathrm{P}}=75, \mathrm{~d}_{1}=0.03, \mathrm{~d}_{2}=0.97, \mathrm{Bi}=1, \operatorname{Pr}=7\right)$

Thermocapillary convection in the cavity for different aspect ratios and Biot numbers $\left(\mathrm{Ma}=60000, \mathrm{Ra}_{\mathrm{L}}=0.1, \mathrm{Ra}_{\mathrm{P}}=0.075, \mathrm{~d}_{1}=0.03\right.$, $\left.\mathrm{d}_{2}=0.97, \operatorname{Pr}=7\right)$

Streamlines and temperature variation in the cavity for different aspect ratios $\left(\mathrm{Ma}=60000, \mathrm{Ra}_{\mathrm{L}}=0.1, \mathrm{Ra} \mathrm{a}_{\mathrm{P}}=0.0055, \mathrm{~d}_{1}=\mathrm{d}_{2}=0.5, \mathrm{Bi}=1\right.$, $\mathrm{Pr}=7$ )

Buoyancy convection in the cavity for different aspect ratios $\left(\mathrm{Ra}_{\mathrm{L}}=1000, \mathrm{Ma}=1, \mathrm{Bi}=1, \operatorname{Pr}=7\right)$

Buoyancy convection in the cavity $(\mathrm{Ma}=1, \mathrm{AR}=2, \mathrm{Bi}=1, \operatorname{Pr}=7)$

Thermocapillary convection in the cavity for different aspect ratios $\left(\mathrm{Ma}=60000, \mathrm{Ra}_{\mathrm{L}}=1, \mathrm{Bi}=1, \operatorname{Pr}=7\right)$

numbers $\left(\mathrm{Ra}_{\mathrm{L}}=1, \mathrm{~d}_{1}=\mathrm{d}_{2}=0.5, \mathrm{AR}=2, \mathrm{Bi}=1, \operatorname{Pr}=7\right)$

Thermocapillary convection in the cavity for different aspect ratios $\left(\mathrm{Ra}_{\mathrm{L}}=1, \mathrm{~d}_{1}=\mathrm{d}_{2}=0.5, \mathrm{Bi}=1, \operatorname{Pr}=7\right)$

Thermocapillary convection in the cavity for different thickness $d_{1}$ 46 and $d_{2}\left(\operatorname{Ra}_{L}=1, A R=4, B i=1, \operatorname{Pr}=7\right)$ 
Figure 17 Combined thermocapillary and buoyancy convection in the cavity $\left(\mathrm{Ma}=60000, \mathrm{Ra}_{\mathrm{L}}=1000, \mathrm{Bi}=1, \mathrm{Pr}=7\right)$

Figure 18 Combined thermocapillary and buoyancy convection in the cavity $\left(\mathrm{Ra}_{\mathrm{L}}=1000, \mathrm{AR}=2, \mathrm{Bi}=1, \mathrm{Pr}=7\right)$

Figure 19 Buoyancy convection velocity plot with a cut on the Z-axis at 50 0.25 , displayed in the $X-Y$ plane $\left(R a_{L}=1000, M a=1, d_{1}=d_{2}=0.5\right.$, $\mathrm{AR}=4, \mathrm{Bi}=1, \mathrm{Pr}=7$ )

Figure 20 Comparison of two and three-dimensional buoyancy convection $\left(\mathrm{Ra}_{\mathrm{L}}=1000, \mathrm{Ma}=1, \mathrm{AR}=4, \mathrm{Bi}=1, \mathrm{Pr}=7\right)$

Figure 21 Thermocapillary convection velocity plot with a cut on the $Z$-axis 52 at 0.25 , displayed in the $\mathrm{X}-\mathrm{Y}$ plane $\left(\mathrm{Ma}=60000, \quad \mathrm{Ra}_{\mathrm{L}}=1\right.$, $\mathrm{d}_{1}=\mathrm{d}_{2}=0.5, \mathrm{AR}=4, \mathrm{Bi}=1, \operatorname{Pr}=7$ )

Figure 22 Comparison of two and three-dimensional thermocapillary convection $\left(\mathrm{Ma}=60000, \mathrm{~d}_{1}=\mathrm{d}_{2}=0.5, \mathrm{AR}=4, \mathrm{Bi}=1, \mathrm{Pr}=7\right)$

Figure 23 Combined thermocapillary and buoyancy convection velocity plot 55 with a cut on the $\mathrm{Z}$-axis at 0.25 , displayed in the $\mathrm{X}-\mathrm{Y}$ plane $\left(\mathrm{Ma}=60000, \mathrm{Ra}_{\mathrm{L}}=1000, \mathrm{~d}_{1}=\mathrm{d}_{2}=0.5, \mathrm{AR}=4, \mathrm{Bi}=1, \mathrm{Pr}=7\right)$

Figure 24 Comparison of two and three-dimensional combined buoyancy 56 and thermocapillary convection $\left(\mathrm{Ma}=60000, \quad \mathrm{Ra}_{\mathrm{L}}=1000\right.$, $\mathrm{d}_{1}=\mathrm{d}_{2}=0.5, \mathrm{AR}=4, \mathrm{Bi}=1, \mathrm{Pr}=7$ )

Figure 25 Thermocapillary convection velocity plot with a cut on the $\mathrm{Z}$-axis 58 at 0.25 , displayed in the $X-Y$ plane $\left(\mathrm{Ma}=60000, \mathrm{Ra}_{\mathrm{L}}=0.1\right.$, $R a_{P}=0.0055, d_{1}=d_{2}=0.5, A R=2, B i=1, \operatorname{Pr}=7$ )

Figure 26 Comparison of two and three-dimensional thermocapillary 59 convection $\left(\mathrm{Ma}=60000, \mathrm{Ra}_{\mathrm{L}}=0.1, \mathrm{Ra}_{\mathrm{P}}=0.0055, \mathrm{~d}_{\mathrm{l}}=\mathrm{d}_{2}=0.5, \mathrm{AR}=2\right.$, $\mathrm{Bi}=1, \operatorname{Pr}=7$ )

Figure 27 Thermocapillary convection with evaporation in the liquid layer $\left(\mathrm{Ma}=60000, \mathrm{Ra}_{\mathrm{L}}=1, \mathrm{Pr}=7\right.$ )

Figure 28 Comparison of thermocapillary convection with and without 64 evaporation in the liquid layer $\left(\mathrm{Ma}=60000, \mathrm{Ra}_{\mathrm{L}}=1, \mathrm{~d}_{1}=\mathrm{d}_{2}=0.5\right.$, Pr=7) 
Figure 29 Comparison of $U$ component of velocity on vertical median of cavity for thermocapillary convection with and without evaporation $\left(\mathrm{Ma}=60000, \mathrm{Ra}_{\mathrm{L}}=1, \mathrm{~d}_{1}=\mathrm{d}_{2}=0.5, \mathrm{Pr}=7\right)$

Figure 30 Thermocapillary convection with evaporation in the liquid layer for different Marangoni numbers $\left(\mathrm{Ra}_{\mathrm{L}}=1, \mathrm{~d}_{1}=\mathrm{d}_{2}=0.5, \mathrm{AR}=2, \mathrm{Pr}=7\right)$

Figure 31 Thermocapillary convection with evaporation in the liquid layer 67 for different Marangoni numbers $\left(R a_{1}=0, d_{1}=d_{2}=0.5, A R=2, P r=7\right.$ )

Figure 32 Comparison of $U$ component of velocity on vertical median of cavity for thermocapillary convection with evaporation $\left(\mathrm{Ra}_{\mathrm{L}}=1\right.$, $\mathrm{d}_{1}=\mathrm{d}_{2}=0.5, \mathrm{AR}=2, \mathrm{Pr}=7$ ) 


\section{NOMENCLATURE}

AR $\quad$ Aspect ratio $=\frac{H}{L}$

Bi Biot number

Ca Capillary number

$\mathrm{C}_{\mathrm{p}} \quad$ Specific heat at constant pressure

$\mathrm{d}_{1} \quad$ Liquid layer thickness

$\mathrm{d}_{2} \quad$ Porous layer thickness

Da Darcy number $=\frac{\kappa}{L^{2}}$

g Gravitational acceleration

G Non-dimensional overall thermal conductivity

$\mathrm{H} \quad$ Length of cavity in horizontal X-direction

$h_{l v} \quad$ Latent heat of vapourization

$i \quad$ Liquid-vapour interface surface

$\mathbf{k}_{\mathbf{f}} \quad$ Conductivity of the fluid

$\mathrm{k}_{\mathbf{s}} \quad$ Conductivity of the solid glass beads

L Characteristic length of cavity in $Y$-direction $=d_{1}+d_{2}$

Ma Marangoni number $=\frac{|\gamma| \cdot\left(T_{H}-T_{C}\right) \cdot L}{\alpha \cdot \mu}$

$\mathrm{Nu} \quad$ Average Nusselt number $=\frac{h \cdot L}{k}$

p Pressure

P Non-dimensional pressure $=\frac{p \cdot L}{\mu \cdot u_{o}}$

Pr Prandtl number $=\frac{v}{\alpha}$

$q \quad$ Heat flux

$\mathrm{Ra}_{\mathrm{L}} \quad$ Liquid layer Rayleigh number

Rap Porous layer Rayleigh number 
Re Reynolds number $=\frac{\rho \cdot u_{o} \cdot L}{\mu}$

T Temperature

$\Delta \mathrm{T}$ Temperature difference between lateral walls equal to unity $=T_{H}-T_{C}$

$u \quad$ Velocity component in the $\mathrm{x}$ direction

$\mathrm{U} \quad$ Non-dimensional velocity component in the $\mathrm{X}$ direction $=\frac{u}{u_{o}}$

$u_{0} \quad$ Reference velocity $=\sqrt{g \cdot \beta_{T} \cdot \Delta T \cdot L}$

$v \quad$ Velocity component in the y direction

$\mathrm{V} \quad$ Non-dimensional velocity component in the $\mathrm{Y}$ direction $=\frac{v}{u_{0}}$

$\bar{V} \quad$ Volume

$w \quad$ Velocity component in the $\mathrm{z}$ direction

$\mathrm{W} \quad$ Non-dimensional velocity component in the $Z$ direction $=\frac{w}{u_{0}}$

\section{Greek symbols}

$\alpha \quad$ Thermal diffusivity $=\frac{k_{f}}{\rho \cdot\left(C_{p}\right)_{f}}$

$\beta_{\mathrm{T}} \quad$ Thermal volume expansion

$\gamma \quad$ Surface tension gradient

$\theta \quad$ Non-dimensional temperature $=\frac{T-T_{C}}{T_{H}-T_{C}}$

$\Delta \theta \quad$ Temperature difference between two plot contours

$\kappa \quad$ Permeability

$\mu \quad$ Dynamic viscosity

$v \quad$ Kinematic viscosity

$\rho$ Density

$\sigma \quad$ Stress 
$\sigma_{\mathrm{m}} \quad$ Non-dimensional surface tension

$\phi \quad$ Porosity

$\Delta \Psi \quad$ Streamline difference between two plot contours

\section{Subscripts}

$\begin{array}{ll}s & \text { Solid glass beads } \\ \text { C } & \text { Cold } \\ e & \text { effective } \\ f & \text { Fluid } \\ \text { H } & \text { Hot } \\ \text { I } & \text { Liquid-porous interface } \\ l & \text { Liquid phase } \\ n & \text { Normal direction to liquid-vapour interface } \\ \text { o } & \text { Reference } \\ t & \text { Tangential direction to the liquid-vapour interface } \\ v & \text { Vapour phase }\end{array}$




\section{CHAPTER 1 \\ Introduction and Literature Review}

\subsection{Introduction}

The study of thermal convection of fluids in porous media is important and has many applications. Many processes in nature involve transport in porous media. An important example is the world's supply of oil, which formed and is stored in porous rock formations. The movement of fluids through porous media is also common in many industrial processes. As an example, distillation and absorption columns are often filled with beads in a variety of shapes that serve to form a porous matrix. The related problem of a liquid layer overlying a porous layer is also found in many environmental and engineering applications. This configuration is evident in water reservoirs, underground storage tanks, and solid matrix heat exchangers to name a few. An extensive collection of work devoted to the area of convection in porous media is documented in the book by Nield et al. [1]. They define a porous medium as a material consisting of a solid matrix with an interconnected void. The void allows the flow of a fluid through the material. Examples of porous media are beach sand, rye bread or glass beads. The porosity $\phi$ is defined as the fraction of the total volume of the medium that is occupied by void space, or the liquid in this case, $\phi=\frac{\bar{V}_{f}}{\bar{V}} . \bar{V}$ is the total volume defined by $\bar{V}=\bar{V}_{f}+\bar{V}_{s}$, where the subscript $f$ denotes the liquid and $s$ the solid beads. This would make 1- $\phi$ the fraction that is occupied by the solid beads. It is important to note that the volume average is applied to the mass, momentum and energy conservation equations solved in the porous medium. Nield et al. [1] also point out that for natural media the porosity does not normally exceed 0.6 . For solid spheres, such as glass beads, the porosity can vary between 0.2545 and 0.4764 .

For the onset of thermal convection in fluids, it is well known that two mechanisms are responsible. The buoyancy (Rayleigh-Benard) effect is the principal mechanism in 
problems where gravity is taken into consideration. The variation of mass density with temperature is the process that drives the flow. However, in micro-gravity the predominant mechanism responsible for convective motion is the thermocapillary Marangoni effect, which is caused by surface tensions. As is noted by Carey [2], the surface tension at the interface between a liquid and a vapour phase varies with temperature in the liquid. Consequently, if the temperature varies over the interface, the interfacial tension will be non-uniform. This results in the liquid near the interface in regions of low surface tension being pulled towards regions of higher surface tension. If the temperature variation is maintained, a steady flow pattern may be established. As defined, in order for Marangoni convection to begin, there has to be an applied temperature gradient. This can either be applied at the bottom of a cavity or at the side, in which case it would be called a laterally heated cavity.

Due to the occurrence of liquid evaporation in experiments, the effect of evaporation on thermocapillary convection becomes very important for a more complete understanding of these processes. Evaporation is the process in which a substance undergoes a state change from liquid to gas, as molecules leave the surface of the liquid and go into the vapour phase. Liquid-vapour phase change processes, such as evaporation, play a vital role in many technological applications. These processes are encountered in power and refrigeration cycles, in petroleum and chemical processing, liquefaction of nitrogen and during evaporation or precipitation of water in the earth's atmosphere to name a few. Carey [2], also points out the application for thermal control in spacecraft environments via a compact evaporator.

Following is the literature review that shows the developments in recent years in the area of the onset of thermo-convection in fluid and porous layers and the effects of evaporation.

\subsection{Onset of Convection in Liquid and Porous Cavity}

Nearly four decades ago, Beavers and Joseph [3] studied by experiment the boundary 
conditions at a naturally permeable wall. The experiment was designed to examine the nature of tangential flow in the boundary region of a fluid-porous interface. A twodimensional Poiseuille flow above a fluid saturated porous block was used to demonstrate the value of the velocity at the interface. It was postulated that the slip velocity at the fluid-porous interface differs from the mean filter velocity within the permeable material, that shear effects are transmitted into the body of the material through a boundary-layer region and that the slip velocity for the free fluid is proportional to the shear rate at the permeable boundary. They found that the slip coefficient was dependent on the structure of the material at the interface. Their experiments have also shown that the flow of a viscous fluid over a porous material induced a boundary layer region within the material. The effect of the boundary layer can be successfully replaced with a slip velocity proportional to the exterior velocity gradient. This is the adopted condition for the interface boundary in many experiments that followed at future dates.

It is known that, when a porous layer is underlying a fluid layer, the critical condition for the onset of convection when the system is being heated from below is considerably different from that of the porous layer alone. The onset of convection in a fluid layer overlying a porous medium was first theoretically treated and published by Nield [4]. Up to that point, convection in a single fluid layer or porous layer was investigated. Nield applied linear stability analysis to superposed fluid and porous layers with uniform heating from below and general boundary conditions. However, since no experimental data was available, the solution was restricted to the case of constant-flux thermal boundary conditions. The Marangoni effect was also taken into account at the deformable upper surface, and it was assumed that only non-oscillatory convection occurs. In the fluid region, the Oberbeck-Boussinesq equations were applied, and in the porous region, the steady-state Darcy-Oberbeck-Boussinesq equations. The boundary conditions applied for the perturbation variables included: no mass flux across the boundaries, continuity of tangential and normal stress at the upper boundary, continuity of normal velocity, normal stress, temperature and heat flux at the interface, and the conditions of Beavers and Joseph [3] relating the shear in the fluid to the slip velocity at the interface. The result of the analysis is a tenth-order system of differential equations 
and ten boundary conditions that form a standard eigenvalue problem. The Rayleigh number can be regarded as the eigenvalue and the non-dimensional horizontal wavenumber $a$ as a parameter. For the stability criterion, the Rayleigh number must be minimized as a function of $a$. The solution for constant heat flux boundary conditions was obtained and checked against known results for special cases such as: viscous fluid between one rigid and one free boundary, viscous fluid between two free boundaries, porous medium between one impermeable boundary and one boundary at constant pressure, and a porous medium between two impermeable boundaries. The results obtained for the constant flux case were also useful for estimating the stability criterion for more general thermal boundary conditions when the critical wave-number is no longer zero.

Chen and Chen [5] have also used linear stability analysis to investigate the hydrodynamic problem of the onset of finger convection in a horizontal porous layer underlying a fluid layer in the directional solidification of concentrated alloys. However, they used a shooting method to solve the eigenvalue problem. They began by first considering the thermal convection problem as a check and in the process uncovered some aspects overlooked by previous investigators. They found that, at low depth ratios (the ratio of the fluid layer depth to the porous layer depth), the marginal stability curve had a bimodal nature and depicted two relative minima. For a depth ratio of 0.10 , the convection pattern was dominated by the porous layer. This changed to that of the fluid layer as the depth ratio was increased to 0.13 . For values greater then 0.13 , the flow remained in the fluid layer, and at values greater then 0.5 , the velocity in the porous layer was essentially zero. The critical fluid Rayleigh number for a Bernard problem with a fixed or a free boundary is known to be 1100 [5], with the porous interface behaving more like a free boundary than a fixed one. In the salt finger case, with a fixed thermal Rayleigh number, as the depth ratio increases, the critical salt Rayleigh number first decreases and upon reaching a minimum increases. This phenomenon is again due to the fact that, at a small depth ratio, convection is dominated by the porous layer, and at a larger, ratio it switches to the fluid layer. 
To verify their theoretical results, Chen and Chen [6] also performed a series of experiments. They once again showed that the fluid layer dominated convection changes to porous layer dominated convection as the thickness ratio decreases.

Kim and Choi [7], further investigated the onset of buoyancy-induced convection and diffusive phenomena between porous and overlying fluid layers heated from below. They focused on the critical Rayleigh number and the corresponding number of cells, and defined the depth ratio $\mathrm{d}$, as the thickness of the liquid layer to that of the porous layer. In the supercritical regime, when $d>0.12$, the convection was limited to the fluid layer with conduction dominating the heat transfer in the porous layer. The number of recirculating cells was shown to increase as the Rayleigh number increased, which in turn caused a larger Nusselt number.

Desaive et al. [8] studied the problem of buoyancy instability in a liquid with a free surface overlying a porous layer heated from below. They described the porous medium using Brinkman's model and determined the corresponding linear stability equations. The role of a free surface whose surface tension is temperature dependent was also examined, which makes their stability analysis unique. They found that for the pure thermocapillary condition, the critical depth ratio $d$, which in essence was the thickness of the porous layer, is equal to 0.96 . If $d$ is below the critical value, the thermocapillary flow will dominate the liquid layer without penetrating to the porous layer. For the pure buoyancy condition, this critical value was found to be equal to 0.90 , above which the buoyancy convection dominated the entire cavity. The critical Rayleigh and Marangoni numbers for the onset of convection were also determined.

The effects of interaction between Rayleigh and Marangoni convection in a system of a liquid layer on top of a porous layer with bottom heating was examined by Saghir et al. [9]. They considered several cases. In the first case, the upper cavity wall was rigid and buoyancy convection was studied. In the second case, the liquid layer had a free surface and the interaction between Marangoni and Rayleigh convection was investigated for different thicknesses of liquid and porous layers. The full Navier-Stokes equations were 
solved for the fluid layer, and the Brinkman model was used for the porous layer. From previous studies it was noted that in the porous medium the fluid remains stationary below the first critical porous Rayleigh number $R a_{P}=4 \pi^{2}$. The flow starts beyond the first critical Rayleigh number and oscillatory flow starts when the second $R a_{P}$ number is above 390. For the fluid layer, it was noted that the critical fluid Rayleigh number $R a_{C}$ was around 1732, and the flow would become oscillatory when the fluid Rayleigh number was above $1 \times 10^{5}$. For the buoyancy convection case, it was found that for equal liquid-porous layer thickness, the convection was confined to the liquid layer. Decreasing the liquid layer to the ratio of $1 / 11$ of the total cavity layer, forced the convection to move to the porous layer. For the combined buoyancy and Marangoni convection, it was found that the Marangoni convection enhanced the flow in the liquid layer. There was also an increase in the Nusselt number in the presence of Marangoni convection.

Villers and Platten [10], studied the convection in liquid acetone due to coupled buoyancy and Marangoni effects. The acetone was subjected to a horizontal temperature difference in a laterally heated cavity. They performed several experiments and ran matching numerical simulations. Different aspect ratios, Rayleigh numbers and Marangoni numbers were considered in the study. Results from both methods confirmed the existence of three states: mono-cellular steady states, multi-cellular steady states and spatio-temporal structures for higher values of the control parameter.

The effect of interaction between Rayleigh and Marangoni convection in a superposed system but with a lateral heating condition was closely examined by Saghir et al. [11]. The convection patters were studied for various ratios of liquid to porous layer as well as various non-dimensional parameters. With equal liquid-porous layer thickness for the Marangoni convection, it was found that as the Marangoni number increases, the formation of multi-cells also increases in the liquid layer. As a result, the flow does not penetrate into the porous layer. A similar result was found for buoyancy convection. With increasing Rayleigh number, the flow did not penetrate into the porous layer. 
However, when the thickness of the liquid layer was decreased, the flow penetrated into the porous cavity even for a small Marangoni number. The same conclusion was observed for buoyancy convection. This numerical study showed the sensitivity of the thickness and non-dimensional parameters with respect to Rayleigh and Marangoni convection.

A three-dimensional finite rectangular container was considered by Dauby and Lebon [12]. The cavity included rigid lateral walls with a realistic no-slip condition and the free surface of the liquid layer was assumed to be non-deformable. They studied thermocapillary convection with the free surface subjected to a temperature-dependent surface tension. Both the linear and non-linear problems were considered. For the linear problem, a spectral Tau method [12] was used to determine the critical Marangoni number and the convective pattern as functions of the aspect ratios of the container. The dependence of the results on non-zero Rayleigh and Biot numbers was examined. The non-linear problem was studied by reducing the dynamics of the system to the dynamics of the most unstable modes of convection. It was found that due to the presence of the rigid walls, the convective pattern above the threshold may be quite different from that predicted by the linear approach.

Pattern formation in thermo-convection when a horizontal fluid layer is heated from below has also received much interest in the last decade. Generally, the motion that appears above the stability threshold is well structured and a regular pattern of convective cells may be observed. The geometrical nature of the convective cells depends greatly on the mechanism that causes the instability. The main results on this subject are outlined next.

Schatz et al. [13], have focused on the onset of surface-tension driven Marangoni convection in a fluid layer heated from below. Their experiments with shadowgraph visualization revealed a sub-critical transition to a hexagonal convection pattern in thin liquid layers that have a free surface. 
Parmentier et al. [14], have taken on the difficult task of performing a non-linear theoretical analysis of coupled buoyancy and capillary thermo-convection. They studied the thermo-convective instability in an infinite horizontal fluid layer heated from below. The influence of the Prandtl number and Biot number was emphasized. For buoyancy convection only, rolls were observed. The situation became more complex when capillary effects were present. It was observed that a hexagonal cell structure was preferred at the linear threshold. For higher thermocapillary forces, the size of the region where hexagons were stable was larger. It was also shown that the direction of motion inside the hexagons was directly linked to the value of the Prandtl number. For $P r>$ 0.23, the fluid moved upwards at the center of the hexagons, but for $\operatorname{Pr}<0.23$, the fluid motion was inverted.

The possibility of the occurrence of square structures in gravitational and capillary thermo-convection was also investigated by Regnier et al. [15]. They showed that square cells occurred when the instability was mainly capillarity driven and the fluid layer was thin enough.

\subsection{Evaporation in Liquid}

In recent years, there has been an increased interest in the phenomena of evaporation, and its effects have been studied for various physical and engineering applications. For example, in the spin-coating process, Haas and Birnie [16] showed evaporation to cause thermocapillary instability within the coating solution. This instability drove the convective flows that resulted in non-uniform coatings. It has also been confirmed that Marangoni instability induced convection can and does occur in the droplet evaporation process [17-19]. A lot of focus on the evaporation phenomena has also been given by Ward and his colleagues [20-22]. They performed several experiments to study the conditions existing at the liquid-vapour interface during evaporation and these are outlined next. 
Recently, it has been noticed that there is a strong disagreement with the predictions from classical kinetic theory with past measurements of the temperature profile across the liquid-vapour interface of an evaporating liquid. However, the previous measurements in the vapour phase were made within 27 mean free paths of the interface. To determine if the disagreement could be resolved Fang and Ward [20] performed a series of experiments with temperature measurements as close as one mean frec path of the interface of an evaporating liquid. They also studied higher rates of evaporation. When the system was brought to a steady state with a constant evaporation rate, the temperature was noted on the center line of the evaporation chamber with thermocouples and a positioning micrometer. Near the interface, the temperature was measured at approximately $0.1 \mathrm{~mm}$ intervals. Classical kinetic theory does specify that sharp changes in the temperature can occur near the interface. From the results, it is shown that in fact it is the higher-energy molecules that escape the liquid during evaporation. A discontinuity is noted in the temperature profile across the interface but in the opposite direction to that predicted by classical kinetic theory and at a much larger magnitude. The highest difference in temperature between the vapour at the interface and the liquid at the interface occurred in the experiment with the highest evaporation rate and was $7.8^{\circ} \mathrm{C}$.

Ward et al. [21] have conducted steady state experiments to study the interfacial conditions during evaporation or condensation of water. The temperature profiles were measured in the liquid phase near the interface. A layer was found where the temperature was uniform. Deeper in the liquid phase, the temperature assumed a constant linear gradient, indicating the mode of energy transport was by thermal conduction. For the evaporation experiments, the thickness of the uniform temperature layer varied from 0.35 to $0.61 \mathrm{~mm}$ and decreased as the evaporation rate was increased. The existence of the uniform temperature layer and the gradient in the sub-interface region suggests that thermocapillary convection is present in the liquid near the interface. There was also a temperature discontinuity that was measured at the liquid-vapour interface in each of the experiments. In both the evaporation and condensation cases, the interfacial temperature in the vapour was greater than that in the liquid. This suggests that it is primarily the molecules from the high-energy end of the energy distribution that are escaping the liquid 
during the evaporation case. A correlation was also found between the magnitude of the temperature discontinuity and an increase in the evaporation flux.

A further experimental analysis was performed by Ward et al. [22], to observe Marangoni-Benard convection of water, which has previously been difficult to accomplish. This could be due to the fact that earlier analytical studies performed have neglected the flow of the fluid to the interface when determining the criterion for the onset of Marangoni convection. A series of water evaporation experiments were conducted under steady state by having the evaporation rate equal to the syringe pumping rate in the procedure. The temperature was then measured over a range of positions by a thermocouple mounted on a positioning micrometer. The temperature profile was found to have a discontinuity at the interface, in which the vapor temperature at the interface was greater than that in the liquid at the interface. As the evaporation flux was increased, the temperature discontinuity increased as well. It has previously been suggested that this discontinuity results from the higher energy molecules escaping the liquid phase during evaporation. To test for recirculation in the uniform temperature region near the liquid interface, the thickness of the uniform temperature layer was measured. The thickness of the uniform temperature layer was shown to vary with the evaporation rate. Below the uniform layer, the temperature profile indicated that conduction was the primary mode of energy transport. It was concluded that the behavior of the uniform temperature layer is consistent with that of a convecting liquid.

\subsection{Research Objectives}

In this thesis, the aim of the study is to investigate the onset of convection in a twodimensional superposed liquid and porous layer cavity subject to bottom and lateral heating. Based on Desaive et al.'s [8] stability analysis, several cases are considered. First, pure buoyancy, pure thermocapillary and combined buoyancy-thermocapillary convection are analyzed for different porous layer thickness and different aspect ratios for

the bottom heating condition. In the second case, the flow is studied in detail for the lateral heating condition. A second aim of the thesis is to verify that the two-dimensional 
results reasonably predict the convective fluid motion in the case of a three-dimensional cavity with buoyancy, thermocapillary and combined convection.

It is also the intention of this thesis to further study thermocapillary convection in a laterally heated cavity with the phenomenon of phase change, namely evaporation, at the free surface. Two cases are considered. In the first case, Marangoni convection is examined without evaporation, while the second case is examined in the presence of evaporation at the free surface. Different parameters are studied, such as the variation of the liquid-porous thickness, the aspect ratio and the Marangoni number. 



\section{CHAPTER 2 \\ Governing Equations and Numerical Solution}

\subsection{Model Description}

The schematic diagram for this study is illustrated in Figure 1. It presents twodimensional and three-dimensional versions of the model. The system consists of a rectangular cavity split into a liquid layer and a porous layer. The incompressible liquid layer, whose thermal expansion coefficient is $\beta_{T}$, has a height of $d_{l}$ and a width of $H$. The physical properties of the liquid are assumed constant, except for the density in the buoyancy term in the momentum equation. The top wall of the liquid layer is a nondeformable free surface.

The liquid layer overlays a homogeneous and isotropic rectangular porous layer that is saturated with the liquid. It is assumed that the fluid and solid are in thermal equilibrium. The porous matrix has a porosity of $\phi=0.39$, which corresponds to having glass beads of $3.25 \mathrm{~mm}$ in diameter [9]. The Darcy number is set to a constant of $D a=1$ $\times 10^{-5}$ for the duration of the study. The porous layer has the same width of $H$ and a height of $d_{2}$. The total thickness is defined by $L=d_{1}+d_{2}$. The aspect ratio is the width of the cavity to its thickness, denoted by $A R=H / L$, and will be studied in detail throughout the thesis. The gravitational acceleration term is set to act in the negative $y$ direction.

For the three-dimensional model, the cavity is extended by a value of 0.5 , which is half of the height of the cavity, in the positive $Z$-axis direction. For this model, the gravitational acceleration term remains pointing in the negative $\mathrm{Y}$-axis direction. The plane on top of the cavity remains a non-deformable free surface. 
Free Surface

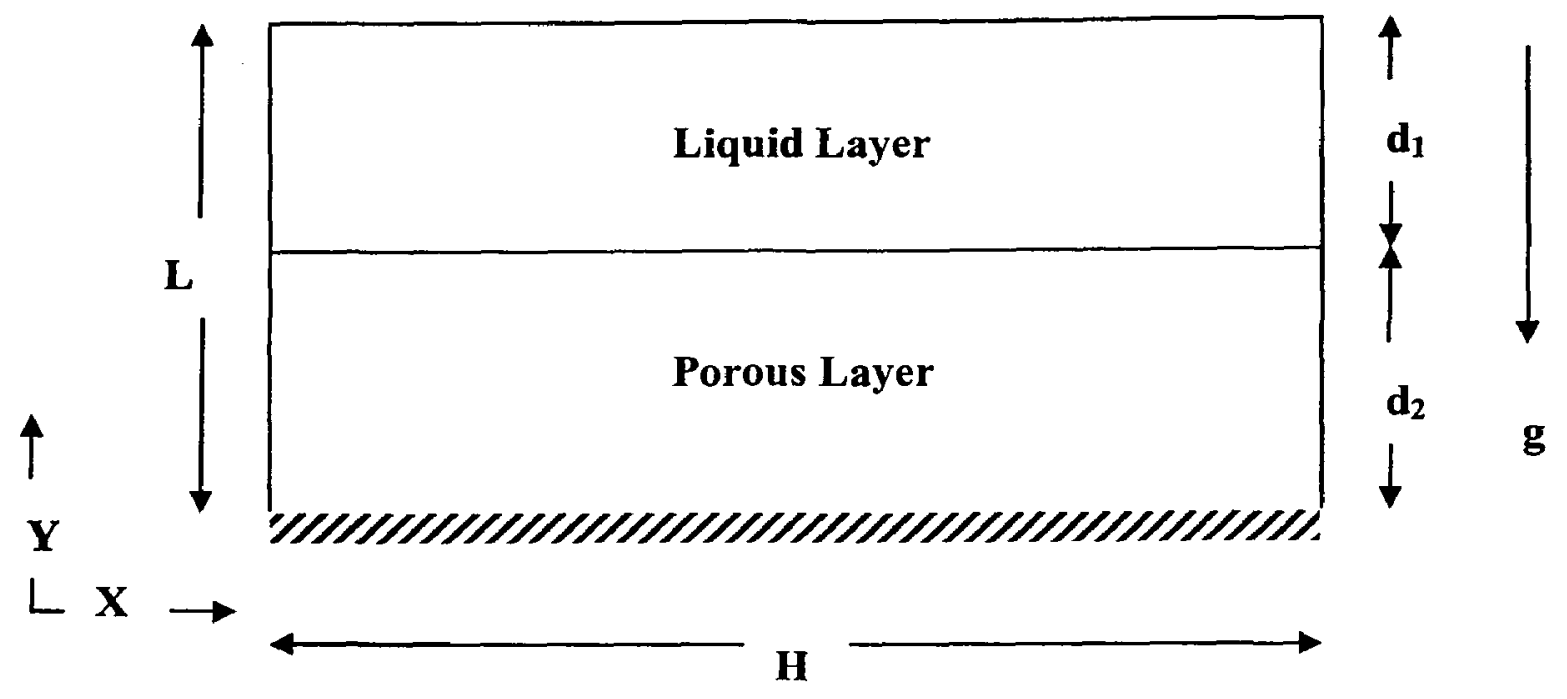

a)

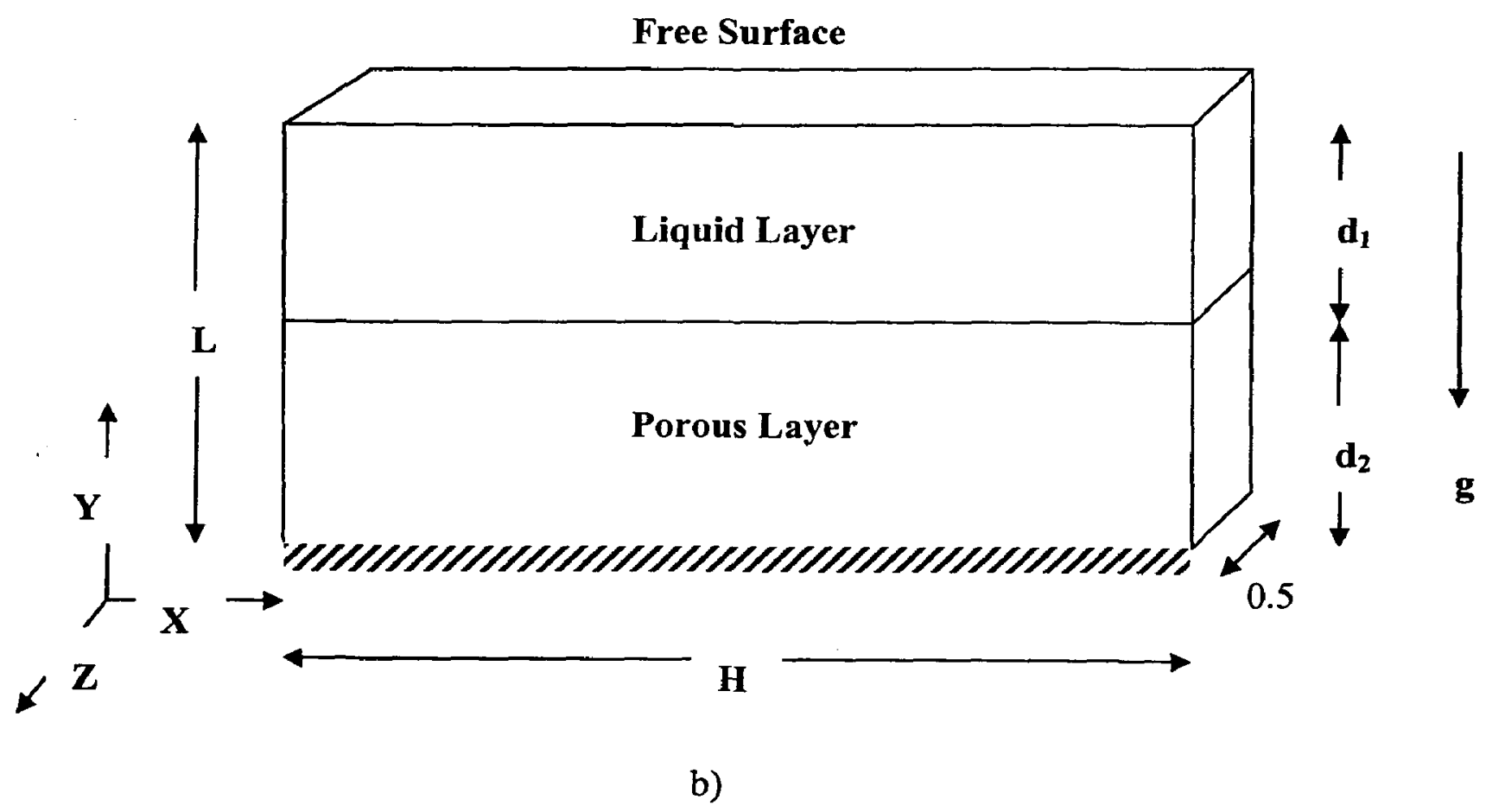

Figure 1 Geometrical model of cavity:

a) Two-dimensional cavity b) Three-dimensional cavity 


\subsection{Liquid Layer Governing Equations}

The flow under consideration is assumed laminar and incompressible. The model is presented in Cartesian coordinates. The complete continuity, momentum balance and energy balance equations are solved simultaneously in order to study the convection patterns. Using the finite element technique, the equations are solved numerically for both the liquid layer and the porous layer of the cavity. Following are the governing equations, boundary conditions and numerical procedure used for the various cases in this study. The equations are presented for the three-dimensional model, but similar equations with the z-term removed are used for the two-dimensional model. The dimensional and non-dimensional equations are explained in detail in Appendix A.

\subsubsection{Continuity Equation}

The equation of continuity is a partial differential equation which represents the conservation of mass for an infinitesimal control volume. The continuity equation for an incompressible fluid is given by:

$$
\left[\frac{\partial u}{\partial x}+\frac{\partial v}{\partial y}+\frac{\partial w}{\partial z}\right]=0
$$

\subsubsection{Momentum Balance Equation}

For the liquid layer, the momentum balance equation is represented by the Navier-Stokes equations. The flow model is Newtonian, incompressible and steady. In the $x$ direction, the principle of conservation of linear momentum dictates that:

$$
\rho_{f} \cdot\left[u \cdot \frac{\partial u}{\partial x}+v \cdot \frac{\partial u}{\partial y}+w \cdot \frac{\partial u}{\partial z}\right]=-\frac{\partial p}{\partial x}+\mu \cdot\left[\frac{\partial^{2} u}{\partial x^{2}}+\frac{\partial^{2} u}{\partial y^{2}}+\frac{\partial^{2} u}{\partial z^{2}}\right]
$$


In the $\mathrm{y}$ direction, the momentum equation is written as follows:

$$
\rho_{f} \cdot\left[u \cdot \frac{\partial v}{\partial x}+v \cdot \frac{\partial v}{\partial y}+w \cdot \frac{\partial v}{\partial z}\right]=-\frac{\partial p}{\partial y}+\mu \cdot\left[\frac{\partial^{2} v}{\partial x^{2}}+\frac{\partial^{2} v}{\partial y^{2}}+\frac{\partial^{2} v}{\partial z^{2}}\right]-\rho_{f} \cdot \beta_{T} \cdot\left(T-T_{C}\right) \cdot g
$$

The Boussinesq approximation in the momentum equation in the $y$-direction allows for modeling of buoyancy effects for an incompressible fluid. The Boussinesq approximation has the following two assumptions: the variations in fluid density affect only the buoyancy term and the fluid density is a function of temperature [23].

Lastly, in the $\mathrm{z}$ direction:

$$
\rho_{f} \cdot\left[u \cdot \frac{\partial w}{\partial x}+v \cdot \frac{\partial w}{\partial y}+w \cdot \frac{\partial w}{\partial z}\right]=-\frac{\partial p}{\partial z}+\mu \cdot\left[\frac{\partial^{2} w}{\partial x^{2}}+\frac{\partial^{2} w}{\partial y^{2}}+\frac{\partial^{2} w}{\partial z^{2}}\right]
$$

In the above equations $u, v$ and $w$ represent the velocities in the $\mathrm{x}, \mathrm{y}$ and $\mathrm{z}$ directions in a Cartesian coordinate system. The $\rho_{f}, p, \mu, \beta_{T}, T$, and $g$ are the density of the fluid, pressure, dynamic viscosity, coefficient of volumetric expansion associated with temperature variations, temperature and gravity, respectively.

\subsubsection{Energy Balance Equation}

The thermal energy conservation equation for an incompressible fluid is expressed as:

$$
\left(\rho \cdot C_{p}\right)_{f} \cdot\left[u \cdot \frac{\partial T}{\partial x}+v \cdot \frac{\partial T}{\partial y}+w \cdot \frac{\partial T}{\partial z}\right]=k_{f} \cdot\left[\frac{\partial^{2} T}{\partial x^{2}}+\frac{\partial^{2} T}{\partial y^{2}}+\frac{\partial^{2} T}{\partial z^{2}}\right]
$$

Where $\rho_{f},\left(C_{p}\right)_{f}$, and $k_{f}$ denote the density, the specific heat at constant pressure and the conductivity of the fluid.

\subsection{Porous Layer Governing Equations}

For the saturated porous matrix, the Brinkman model was used. As noted by Saghir et al. 
[11], at the fluid-porous interface, the boundary conditions are the continuities of velocities, temperatures, normal shear stresses and heat flux. Following are the continuity, momentum balance and energy balance equations for the porous medium.

\subsubsection{Continuity Equation}

Since the flow is incompressible, the continuity equation for the porous layer is also given by:

$$
\left[\frac{\partial u}{\partial x}+\frac{\partial v}{\partial y}+\frac{\partial w}{\partial z}\right]=0
$$

\subsubsection{Momentum Balance Equation}

Darcy was the first to formulate the basic equation of flow in porous media based on the proportionality between the flow rate and the applied pressure difference that was revealed from experiment [1]. Conventionally, Darcy's law was used as the momentum balance equation in a porous medium. However, as noted by Saghir et al. [9], it suffers from mathematical inaccuracy due to the inability to impose a no-slip boundary condition. Consequently, in this study the Brinkman equation is used to represent the momentum equation. In the $\mathrm{x}$ direction, the momentum equation is written as follows:

$$
\frac{\mu \cdot u}{\kappa}=-\frac{\partial p}{\partial x}+\mu_{e} \cdot\left[\frac{\partial^{2} u}{\partial x^{2}}+\frac{\partial^{2} u}{\partial y^{2}}+\frac{\partial^{2} u}{\partial z^{2}}\right]
$$

In the $y$ direction, the momentum equation is represented by:

$$
\frac{\mu \cdot v}{\kappa}=-\frac{\partial p}{\partial y}+\mu_{e} \cdot\left[\frac{\partial^{2} v}{\partial x^{2}}+\frac{\partial^{2} v}{\partial y^{2}}+\frac{\partial^{2} v}{\partial z^{2}}\right]-\rho \cdot \beta_{T} \cdot\left(T-T_{C}\right) \cdot g
$$

Lastly, in the $\mathrm{z}$ direction: 


$$
\frac{\mu \cdot w}{\kappa}=-\frac{\partial p}{\partial z}+\mu \cdot\left[\frac{\partial^{2} w}{\partial x^{2}}+\frac{\partial^{2} w}{\partial y^{2}}+\frac{\partial^{2} w}{\partial z^{2}}\right]
$$

Here, the permeability is denoted by $\kappa$ in the Darcy term on the left hand side of the above equations (7)-(9). The Brinkman form of the momentum equation is suitable when one wants to match a solution in a porous medium and in an adjacent viscous fluid. The Brinkman extension is added as the second term on the right hand side of the above equations.

\subsubsection{Energy Balance Equation}

The energy equation for the porous layer is given by:

$$
\left(\rho \cdot C_{P}\right)_{f} \cdot\left[u \cdot \frac{\partial T}{\partial x}+v \cdot \frac{\partial T}{\partial y}+w \cdot \frac{\partial T}{\partial z}\right]=k_{e} \cdot\left[\frac{\partial^{2} T}{\partial x^{2}}+\frac{\partial^{2} T}{\partial y^{2}}+\frac{\partial^{2} T}{\partial z^{2}}\right]
$$

In addition to the governing equation, the following constitutive thermal relationship is used for the overall thermal conductivity:

$$
k_{e}=\phi+(1-\phi) \cdot \frac{k_{s}}{k_{f}}
$$

The subscript $e$ specifies an effective property for which the value must be set. The effective thermal property is related to the fluid and solid matrix properties by the relation in Equation (11). The subscript $s$ refers to the solid matrix properties while $f$ denotes the fluid properties [23]. In Equation (11), $\mathrm{k}_{\mathrm{e}}, \mathrm{k}_{\mathrm{s}}$, and $\mathrm{k}_{\mathrm{f}}$ are the effective, solid and fluid conductivities, respectively. The porosity is denoted by $\phi$. In general, the dynamic viscosity $\mu$ and the effective dynamic viscosity $\mu_{e}$ are only approximately equal to each other. However, the Brinkman approximation sets the viscosity and the effective viscosity equal to each other. It is also important to note that, in order to maintain consistent boundary conditions in the porous layer, the equations in the fluid and porous medium are all solved in terms of volume averaged quantities. 


\subsection{Non-Dimensional Analysis}

Non-dimensional formulation of the governing equations has many advantages. Scaling the variables and assembling the non-dimensional parameters provides a measure of the importance of the various terms in the equations and identifies the dominant physical phenomena [23]. Equations (1) - (11) were rendered dimensionless by using the following non-dimensional groups:

$$
\begin{aligned}
& U=\frac{u}{u_{o}}, \quad V=\frac{v}{u_{o}}, \quad W=\frac{w}{u_{o}}, \quad X=\frac{x}{L}, \quad Y=\frac{y}{L}, \quad Z=\frac{z}{L}, \\
& P=\frac{p \cdot L}{\mu \cdot u_{o}}, \quad \theta=\frac{T-T_{C}}{T_{H}-T_{C}}, \quad L=d_{1}+d_{2}, \quad u_{o}=\sqrt{g \cdot \beta_{T} \cdot \Delta T \cdot L}
\end{aligned}
$$

Where $U, V, W, X, Y$, and $Z$ are the non-dimensional $\mathrm{x}, \mathrm{y}$ and $\mathrm{z}$ component of velocity, and non-dimensional $\mathrm{x}, \mathrm{y}$ and $\mathrm{z}$ coordinates respectively. $P$ is the non-dimensional pressure term and $\theta$ the non-dimensional temperature term. The characteristic length, temperature and velocity are denoted by $L, T$, and $u_{o}$. During the non-dimensional analysis several other parameters appear, such as the Reynolds number $R e$, the Darcy number $D a$, and the Prandtl number, Pr. The parameters in Equation (12) were used in the analysis, which is fully outlined in Appendix A. The governing equations in their dimensionless form are described in the sections below.

\subsection{Non-Dimensional Liquid Layer Governing Equations}

\subsubsection{Continuity Equation}

$$
\left[\frac{\partial U}{\partial X}+\frac{\partial V}{\partial Y}+\frac{\partial W}{\partial Z}\right]=0
$$

\subsubsection{Momentum Balance Equation}

The Navier-Stokes equations for the $\mathrm{X}, \mathrm{Y}$ and $\mathrm{Z}$ directions are given as follows: 
$\mathrm{X}$ direction:

$$
\operatorname{Re} \cdot\left[U \cdot \frac{\partial U}{\partial X}+V \cdot \frac{\partial U}{\partial Y}+W \cdot \frac{\partial U}{\partial Z}\right]=-\frac{\partial P}{\partial X}+\left[\frac{\partial^{2} U}{\partial X^{2}}+\frac{\partial^{2} U}{\partial Y^{2}}+\frac{\partial^{2} U}{\partial Z^{2}}\right]
$$

Y direction:

$$
\operatorname{Re} \cdot\left[U \cdot \frac{\partial V}{\partial X}+V \cdot \frac{\partial V}{\partial Y}+W \cdot \frac{\partial V}{\partial Z}\right]=-\frac{\partial P}{\partial Y}+\left[\frac{\partial^{2} V}{\partial X^{2}}+\frac{\partial^{2} V}{\partial Y^{2}}+\frac{\partial^{2} V}{\partial^{2} Z}\right]-\operatorname{Re} \cdot \theta
$$

$\mathrm{Z}$ direction:

$$
\operatorname{Re} \cdot\left[U \cdot \frac{\partial W}{\partial X}+V \cdot \frac{\partial W}{\partial Y}+W \cdot \frac{\partial W}{\partial Z}\right]=-\frac{\partial P}{\partial Z}+\left[\frac{\partial^{2} W}{\partial X^{2}}+\frac{\partial^{2} W}{\partial Y^{2}}+\frac{\partial^{2} W}{\partial^{2} Z}\right]
$$

\subsubsection{Energy Balance Equation}

$$
\operatorname{Re} \cdot \operatorname{Pr} \cdot\left[U \cdot \frac{\partial \theta}{\partial X}+V \cdot \frac{\partial \theta}{\partial Y}+W \cdot \frac{\partial \theta}{\partial Z}\right]=\left[\frac{\partial^{2} \theta}{\partial X^{2}}+\frac{\partial^{2} \theta}{\partial Y^{2}}+\frac{\partial^{2} \theta}{\partial Z^{2}}\right]
$$

\subsection{Non-Dimensional Porous Layer Governing Equations}

\subsubsection{Continuity Equation}

$$
\left[\frac{\partial U}{\partial X}+\frac{\partial V}{\partial Y}+\frac{\partial W}{\partial Z}\right]=0
$$

\subsubsection{Momentum Balance Equation}


$\mathrm{X}$ direction:

$$
\left[\frac{1}{D a}\right] \cdot U=-\frac{\partial P}{\partial X}+\left[\frac{\partial^{2} U}{\partial X^{2}}+\frac{\partial^{2} U}{\partial Y^{2}}+\frac{\partial^{2} U}{\partial Z^{2}}\right]
$$

Y direction:

$$
\left[\frac{1}{D a}\right] \cdot V=-\frac{\partial P}{\partial Y}+\left[\frac{\partial^{2} V}{\partial X^{2}}+\frac{\partial^{2} V}{\partial Y^{2}}+\frac{\partial^{2} V}{\partial Z^{2}}\right]-\operatorname{Re} \cdot \theta
$$

$\mathrm{Z}$ direction:

$$
\left[\frac{1}{D a}\right] \cdot W=-\frac{\partial P}{\partial Z}+\left[\frac{\partial^{2} W}{\partial X^{2}}+\frac{\partial^{2} W}{\partial Y^{2}}+\frac{\partial^{2} W}{\partial Z^{2}}\right]
$$

\subsubsection{Energy Balance Equation}

$$
\begin{gathered}
\operatorname{Re} \cdot \operatorname{Pr} \cdot\left[U \cdot \frac{\partial \theta}{\partial X}+V \cdot \frac{\partial \theta}{\partial Y}+W \cdot \frac{\partial \theta}{\partial Z}\right]=G \cdot\left[\frac{\partial^{2} \theta}{\partial X^{2}}+\frac{\partial^{2} \theta}{\partial Y^{2}}+\frac{\partial^{2} \theta}{\partial Z^{2}}\right] \\
G=\phi+(1-\phi) \cdot \frac{k_{s}}{k_{f}}
\end{gathered}
$$

Two model configurations are studied, namely, a bottom heated cavity and a laterally heated cavity illustrated, in Figure 2 and Figure 3. The definitions of the liquid Rayleigh number $R a_{L}$ and the porous Rayleigh number $R a_{P}$ vary, depending on the case studied. The same also applies to the definition of the Reynolds number, which is fully derived in Appendix A [11].

For the bottom heating case, the Reynolds number is defined as: 


$$
\operatorname{Re}=\sqrt{\frac{1}{\operatorname{Pr}} \cdot\left[R a_{L} \cdot\left(1+\frac{d_{2}}{d_{1}}\right)^{3}+\frac{R a_{P}}{D a} \cdot\left(1+\frac{d_{1}}{d_{2}}\right)\right]}
$$

Where the liquid and porous Rayleigh numbers are defined as follows:

$$
R a_{L}=\frac{g \cdot \beta_{T} \cdot d_{1}^{3} \cdot\left(T_{I}-T_{C}\right)}{\alpha \cdot v}, \quad R a_{P}=\frac{g \cdot \beta_{T} \cdot d_{2} \cdot \mathcal{K} \cdot\left(T_{H}-T_{I}\right)}{\alpha \cdot v}
$$

The Darcy number is defined as $D a=\frac{\kappa}{L^{2}}$ and the Prandtl number is defined as $\operatorname{Pr}=\frac{\nu}{\alpha}$. $\alpha$ and $v$ are the thermal diffusivity and kinematic viscosity respectively. The thickness of the liquid layer is represented by $d_{I}$ and the porous layer by $d_{2}$. Here, $T_{H}$ is the hot temperature at the bottom surface of the cavity, $T_{C}$ is the temperature of the surrounding gas near the free surface, and $T_{I}$ is the temperature at the interface between the liquid and porous layers.

For the lateral heating case, the Reynolds number is defined as:

$$
\operatorname{Re}=\sqrt{\frac{R a_{L}}{\operatorname{Pr}} \cdot\left(1+\frac{d_{2}}{d_{1}}\right)^{3}}
$$

Where the liquid and porous Rayleigh numbers are defined as follows:

$$
R a_{L}=\frac{g \cdot \beta_{T} \cdot d_{1}^{3} \cdot\left(T_{H}-T_{C}\right)}{v \cdot \alpha}, \quad R a_{P}=\frac{g \cdot \beta_{T} \cdot d_{2} \cdot \kappa \cdot\left(T_{H}-T_{C}\right)}{v \cdot \alpha}
$$

Here, $T_{H}$ represents the temperature of the right vertical wall also known as the hot surface and $T_{C}$ denotes the temperature of the cold vertical wall of the cavity. These equations also show a clear relationship between the liquid Rayleigh number and the porous Rayleigh number for the lateral heating condition. By assuming that the thickness 
of the porous layer is approximately that of the entire height of the cavity, this relationship can be expressed as:

$$
R a_{P}=R a_{L} \cdot D a \cdot\left(\frac{d_{2}}{d_{1}}\right)^{3}
$$

Each geometrical model also has its own specific boundary conditions depending on the case studied and these are presented next.

\subsection{Model Boundary Conditions}

In order to properly analyze fluid motion, the basic conservation laws have to be applied along with the appropriate boundary conditions on each segment of the boundary. These conditions are shown for the two cases studied, namely, with the bottom heating and lateral heating conditions. The boundary conditions for the evaporation case are also fully outlined in the sections below.

\subsubsection{Bottom Heating Boundary Condition}

Figure 2 shows a diagram of the boundary conditions applied to the cavity with bottom heating. The bottom surface is maintained at a temperature $\theta$, while the two vertical side walls are kept adiabatic. In the porous layer, the velocity components that are normal to the wall surface at the boundary of the cavity are set to zero. The velocity components that are parallel to the wall surface remain free to move to take into account the effects of the porous matrix. However, in the liquid layer, all the components of velocity are set to zero at the boundary of the cavity. At the non-deformable free surface, the velocity component normal to the surface is set to zero, and heat is lost to the surroundings through natural convection. 


$$
\begin{aligned}
& \mathbf{V}=0, \theta=0, \sigma_{m}=\frac{M a}{\operatorname{Re} \cdot \operatorname{Pr}}
\end{aligned}
$$

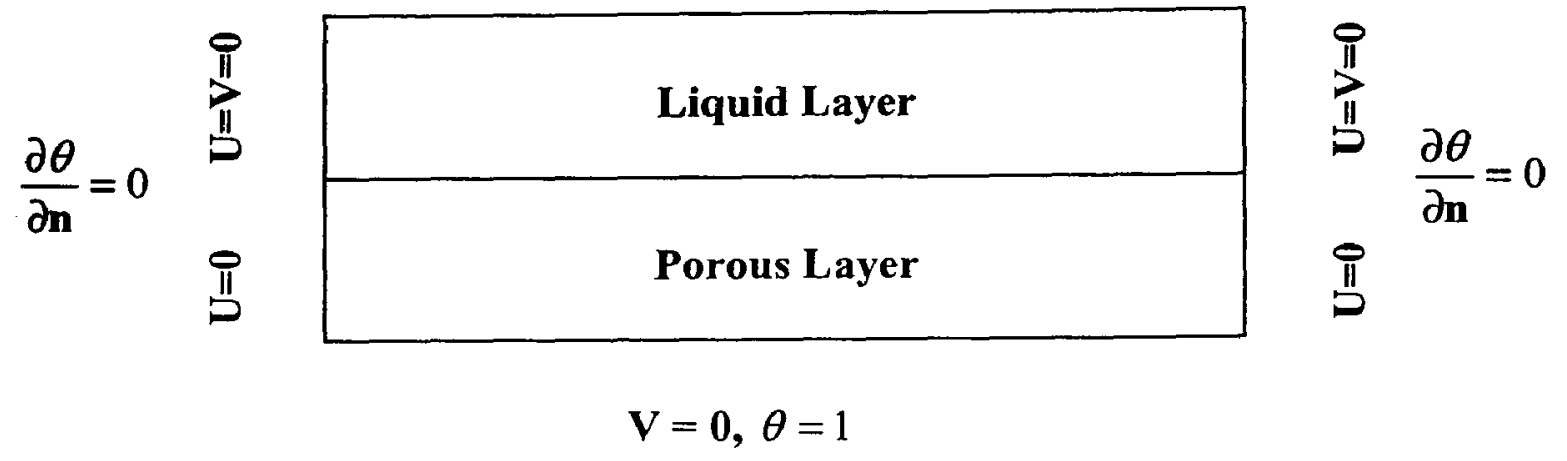

Figure 2 Bottom heating boundary condition

\subsubsection{Lateral Heating Boundary Condition}

In the case of the cavity which is laterally heated, the horizontal temperature gradient is applied parallel to the free surface. The left vertical wall is fixed with the cold temperature $T_{C}$, while the right vertical wall is maintained at a hot temperature $T_{H}$. The bottom surface is insulated, while the top surface of the liquid cavity has a nondeformable free surface through which heat is lost to the surroundings by natural convection. Since Marangoni convection is studied, the heat loss through the free surface is defined by the Biot number, $B i$.

The boundary conditions for the four walls of the cavity are presented in Figure 3. To take into consideration the Marangoni effect, which is the variation of surface tension along the free surface, a boundary condition for the shear stress jump along the liquid-gas interface is included. To account for this, a new non-dimensional surface tension is defined as, $\sigma_{m}=\frac{M a}{\operatorname{Re} \cdot \operatorname{Pr}}$.It is a function of the Marangoni number, $M a=\frac{|\gamma| \cdot\left(T_{H}-T_{C}\right) \cdot L}{\alpha \cdot \mu}$, the Reynolds number defined previously, and the Prandtl number, $\operatorname{Pr}=\frac{\mu \cdot\left(C_{P}\right)_{f}}{k}$. For 
this experiment, a linear variation of surface tension with temperature is specified at the free surface, which is kept at a constant location.

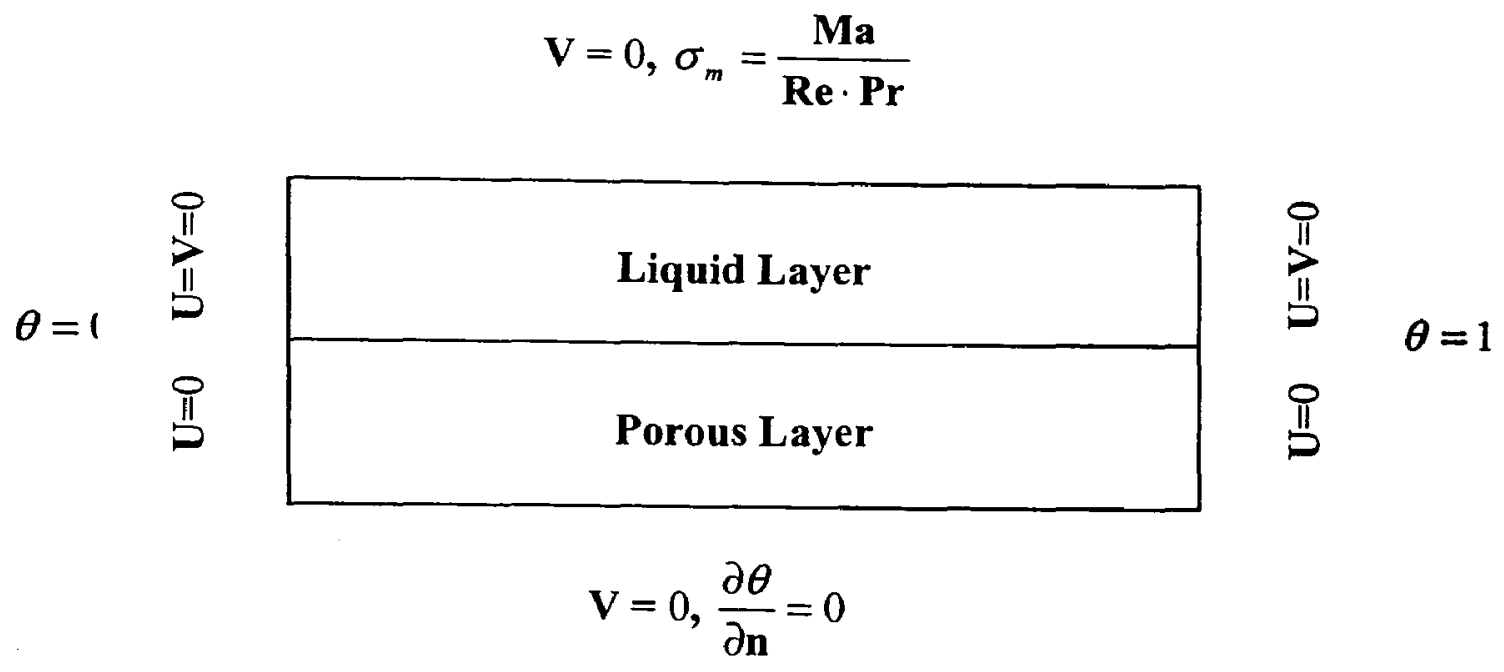

Figure 3 Lateral heating boundary condition

\subsubsection{Evaporation Boundary Condition}

The modeling capabilities in FIDAP 8.7.0 have been enhanced to allow for evaporation at the liquid-vapour interface. This allows for user-defined expressions for saturation temperature and the latent heat of vapourization, as well as modeling of a single phase, in this case, the liquid phase.

For the study of evaporation at the free surface, a laterally heated cavity was utilized as

shown in Figure 4. The right vertical wall was set to the non-dimensional temperature $\theta$ $=1$, and the left vertical wall kept as the cold wall with $\theta=0$. The bottom surface of the cavity was kept adiabatic, and the proper velocity constraints were set on all the cavity walls.

It is also important to specify appropriate boundary conditions at the liquid-vapour interface to assist the solution of the governing equations for heat, mass and momentum transfer in the two fluids on either side of the interface. As noted in Carey [2], at the 
interface, the system must satisfy the principles of conservation of mass, momentum and energy. It should be noted that the general equations are greatly simplified due to the non-deformable surface condition, as well as the modeling of the liquid phase only. The conservation of mass at the interface results in:

$$
\rho_{l} \cdot \mathbf{u}_{l, n}=\rho_{v} \cdot \mathbf{u}_{v, n}
$$

Where the subscripts $l, v$, and $n$ denote the liquid phase, vapour phase and the normal direction, respectively.

Conservation of momentum in the normal direction to the interface, with no interface curvature effects and with negligible interface motion (hence no liquid and vapour momentum terms), results in the following boundary condition:

$$
\sigma_{l, n}=\sigma_{v, n}
$$

Where $\sigma_{t, n}$ and $\sigma_{v, n}$ are the normal stresses in the liquid and vapour phases normal to the interface.

Continuity of tangential stress at the interface, along with the no-slip condition can be written as:

$$
\sigma_{l, t}-\sigma_{v, t}=\frac{d \gamma}{d i}, \quad \mathbf{u}_{l, t}=\mathbf{u}_{v, t}
$$

The surface tension is assumed to vary linearly tangential to the liquid-vapour interface, $i$, to allow for thermocapillary convection.

Lastly, the balance of thermal energy at the interface results in the following condition:

$$
q_{l}-q_{v}=\rho_{l} \cdot \mathbf{u}_{l, n} \cdot h_{l v}
$$


Where $q_{l}$ and $q_{v}$ are the heat fluxes on the liquid and vapour sides, respectively, and $h_{l v}$ is the latent heat of vapourization. It is also assumed that local thermodynamic equilibrium exists at the interface. In addition, a necessary boundary condition for thermal transport in the vapour and liquid regions is a saturation temperature that is applied linearly at the interface.

In FIDAP 8.7.0, equations (29), (30), and (31) are imposed during the solution of the momentum and conservation of mass equations. The saturation temperature condition is imposed during the solution of the energy equation [23].

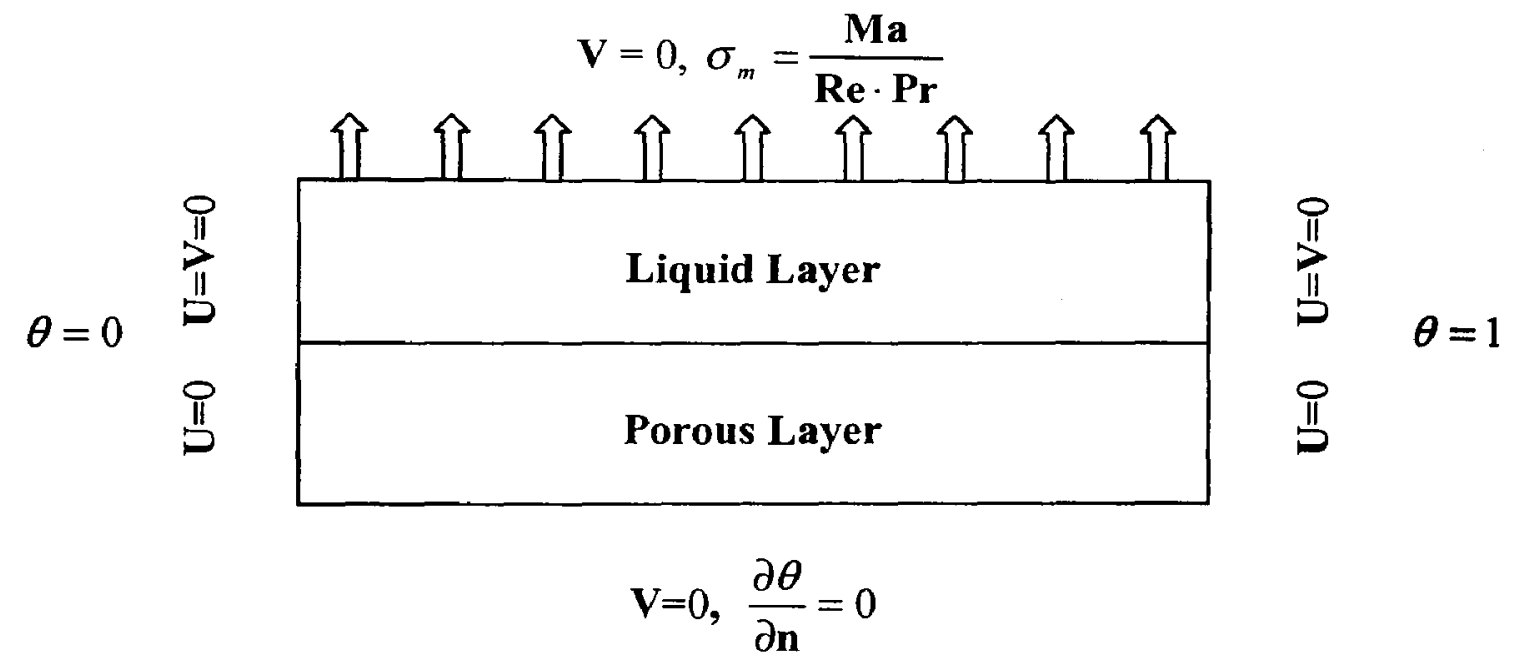

Figure 4 Evaporation boundary condition

\subsection{Numerical Solution Technique}

The fluid dynamics analysis package FIDAP 8.7.0 that uses the finite element method is used in this study. The source code for several cases is outlined in Appendix B.

For free surface problems, FIDAP 8.7.0 utilizes the segregated solver. This is an 
uncoupled method where each degree of freedom is solved separately. To update the free surface during iteration, the normal stress update algorithm is used for cases without evaporation, and the kinematic algorithm for cases with evaporation. In the kinematic update, the normal and tangential stress boundary conditions are satisfied throughout the solution procedure [23].

In post-processing operations, variables such as the stream function, heat fluxes and flow rates can be derived from the numerically computed velocity, pressure and temperature fields. These capabilities are provided by the graphics postprocessor program FIPOST, which can also graphically display the numerical results [23].

\subsubsection{Finite Element Analysis}

The numerical procedure consisted of solving the non-dimensional Equations (13) - (17) and Equations (18) - (23) using the finite element technique. The finite element technique reduces the infinite number of degrees of freedom in a problem to a finite number by solving a system of equations. For the two-dimensional model, the computational domain was divided into many small quadrilaterals. For the threedimensional model, the cavity was divided into brick elements. To achieve a greater accuracy in the results, a finer mesh was applied to the two vertical walls of the rectangular cavity and at the free surface where the driving force of the flow is located and where evaporation takes place in the second case.

The mesh was defined with a finite number of elements, where the variables were evaluated simultaneously. As can be seen in Figure 5, the free surface of the cavity is defined by key-points 3 to 9 , and the fluid-porous layer interface by key-points 2 to 8 . The velocities, temperature and pressure are unknowns and are numerically calculated at each node in the meshed cavity. A linear approximation for the pressure using the penalty method is adopted. The convergence criterion for the iterative solution of symmetric and non-symmetric linear equation systems is $10^{-6}$. Therefore the iterations will continue until an error of $10^{-6}$ is found between two consecutive iterations. 
$6(3,5)$

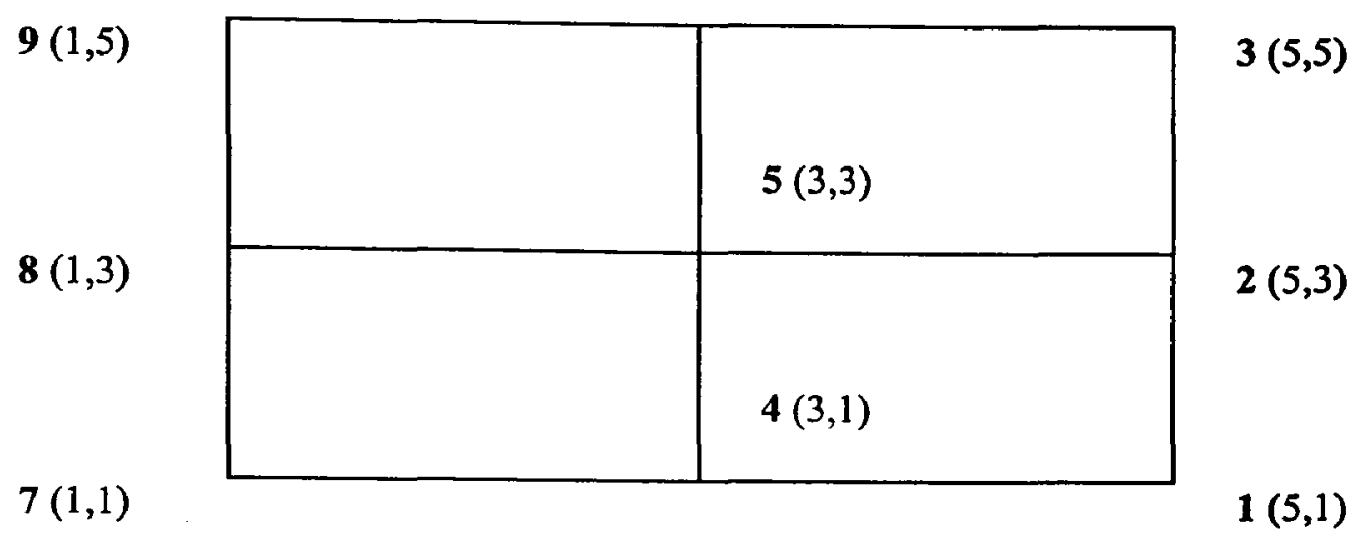

Figure 5 Node numbers for key-points

\subsubsection{Mesh Sensitivity Analysis}

Performing a mesh sensitivity analysis is an integral part of producing accurate, timeefficient and cost-effective results. For this study, the nodal point mesh for the cavity was generated according to the anticipated field variables. Since a lateral heating scheme was employed for much of this study, it was assumed that a high temperature gradient occurs parallel to the free surface. Numerically, it is always wise to have fine nodal spacing in the direction of high gradients, while in directions with smaller gradients much larger nodal spacing may be used. As mentioned before, the mesh is graded so that it is finer near the hot and cold wall, as well as the free surface. For the two-dimensional study, the number of elements in the $\mathrm{Y}$-axis was kept constant at 40. For the mesh sensitivity analysis, the cavity was varied from 40 to 140 elements in the X-direction in increments of 20 . Figure 6 shows some of the different finite element mesh configurations considered. It is evident from this figure how the coarseness of the mesh changes with each trial. For the sensitivity analysis, the two-dimensional pure thermocapillary case with a laterally heated cavity was used to calculate and compare the 
Nusselt number for the various mesh gradients. The case selected had the following parameters: the Marangoni number $M a=60000$, the liquid and porous layer thicknesses $d_{1}=d_{2}=0.5$ and the aspect ratio $A R=4$. The Nusselt number has been chosen for the analysis because it is directly related to the driving force of the physical problem, the temperature gradient. The Nusselt number is calculated at the hot wall and at the cold wall in a similar fashion to that of Kim and Choi [7]. The equations are expressed as follows:

$$
N u_{H}=\left.\int_{0}^{L} \frac{\partial \theta}{\partial Y}\right|_{X=4} \cdot d Y \quad N u_{C}=\left.\int_{0}^{L} \frac{\partial \theta}{\partial Y}\right|_{X=0} \cdot d Y
$$

The overall averaged Nusselt number is:

$$
N u=\frac{N u_{H}+N u_{C}}{2}
$$

The results of the analysis are presented in Table 1, with the Nusselt number listed for each case. It is evident from the table that the Nusselt number is converging to an accuracy of two decimal places after the 100 element point. There is not a significant change between 120 and 140 elements. Thus for the present study a mesh of 120 elements in the $\mathrm{X}$-axis by 40 elements in the $\mathrm{Y}$-axis is selected. The chosen mesh configuration is shown in Figure 6c. Utilizing a finer mesh would result in a longer time being required for convergence without significantly improving the accuracy of the results.

For the three-dimensional model, a similar finite element mesh is adopted in the $\mathrm{X}$ and $\mathrm{Y}$ direction. It should be pointed out that in the Z-direction the cavity has a length of 0.5 as compared to the total height of 1 in the $\mathrm{Y}$-direction for the cavity. Assuming that the driving force of the temperature gradient is still applied in the X-direction, a finite element mesh of 10 elements is chosen for the Z-axis. 

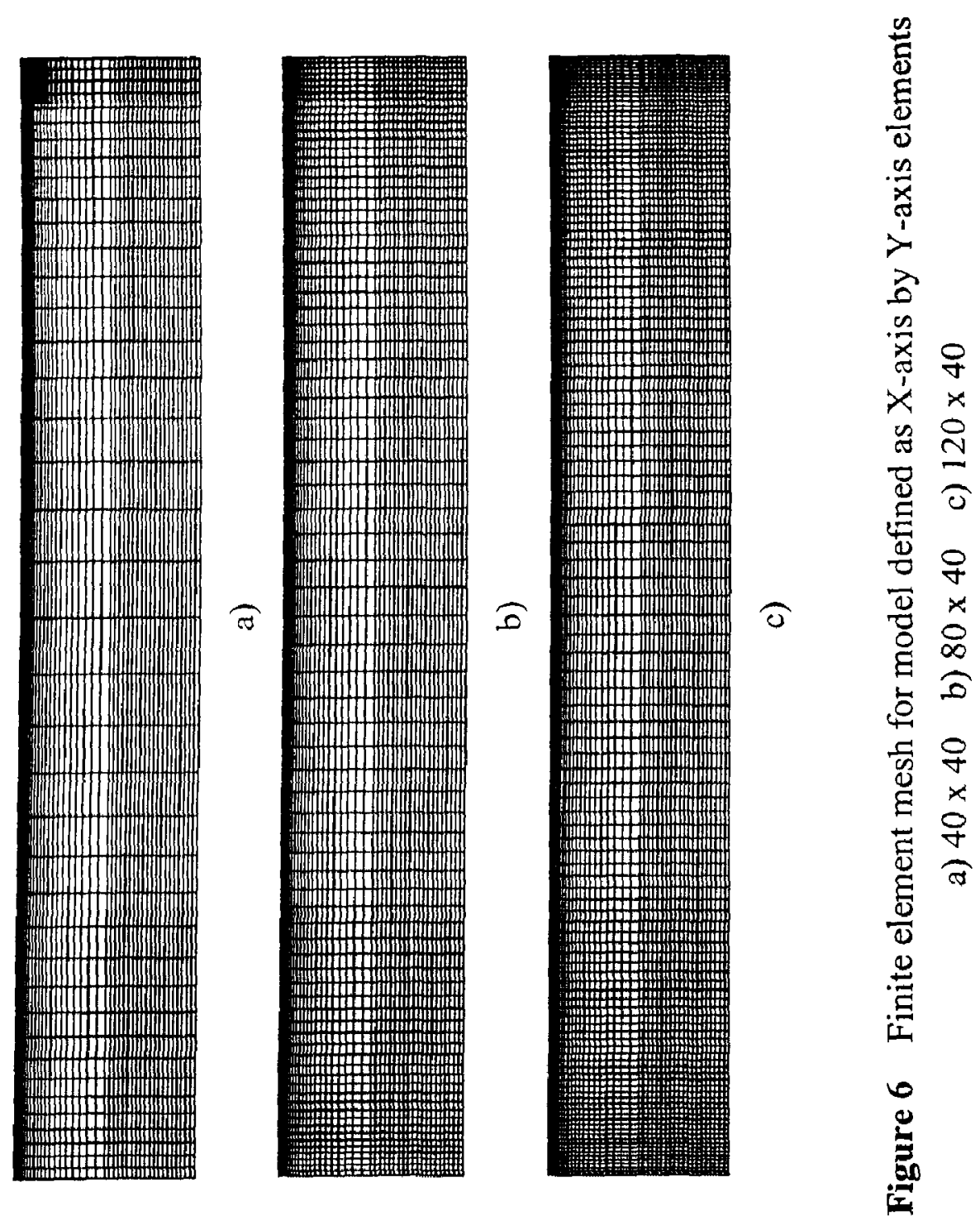

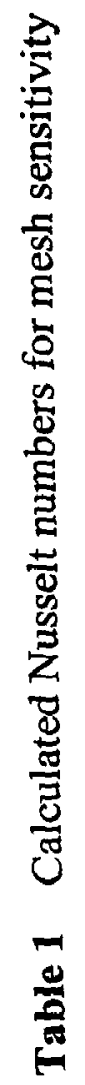





\section{CHAPTER 3 \\ Superposed Liquid and Porous Layer with Adiabatic Free \\ Surface}

\subsection{Two Dimensional Modeling}

To study the patterns of fluid motion, the discipline of fluid mechanics utilizes several different techniques to visualize the flow. One common type of line pattern is a streamline, which is a line everywhere tangent to the velocity vector at any given instant [24]. In this study, to display the results of computed flow fields for the two-dimensional modeling, streamline functions are employed. For two-dimensional incompressible flow, the stream function, $\Psi$, is determined using the following relationships: $U=\frac{\partial \Psi}{\partial Y}$ and $V=-\frac{\partial \Psi}{\partial X}$. The lines of constant $\Psi$ are then used to give the streamline pattern of the flow in the graphical results. The two-dimensional modeling section is broken down into several subsections. Bottom heating and lateral heating conditions are investigated for buoyancy, thermocapillary and combined convection in a cavity with a superposed liquid and porous layer.

\subsubsection{Bottom Heating Buoyancy Convection}

The convection pattern predicted by the linear stability analysis of Desaive et al. [8] for different Biot numbers, $B i=100$ and $B i=1$ is shown in Figure 7 for aspect ratio $A R=1$. Desaive et al.'s analysis showed that the flow switched from convection in the liquid layer to that of the porous layer when the thickness $d_{2}$, was equal to 0.90 . A relationship between the thickness $d_{2}$ and the critical Rayleigh number was also found for Biot numbers that exceeded 100. Therefore, in the first case, the Biot number is set equal to 100. Also, in the present analysis the liquid Rayleigh number, $R a_{L}$ is set equal to 1000 and the porous Rayleigh number, $R a_{P}$ is set equal to 55 , for $d_{2}=0.9$. For $d_{2}=0.97$, the 
liquid Rayleigh number is set to 100 and the porous Rayleigh number is set to 75 .

In the figure, two large convective cells are evident, rotating in opposite directions with the left cell rotating counterclockwise. When the porous layer thickness is equal to the critical value of $d_{2}=0.90$, the center of the cell is in the fluid layer. The main convection is also taking place in the fluid layer, with the flow beginning to penetrate into the porous layer. However, as the porous layer thickness is increased to above the critical value, namely $d_{2}=0.97$, the convection then switches completely to a porous layer dominated flow. When the flow of the $B i=100$ case is compared to that of the $B i=1$ case, it can be seen that the flow becomes weaker with decreasing Biot number. This is also evident in Figure $7 \mathrm{a}$ as the center of the convective cell moves closer to the lateral walls. For the case of $B i=1$ in Figure $7 \mathrm{~b}$, the cell also moves closer to the liquid layer due to the lower Biot number. According to Desaive et al.'s analysis, the critical Rayleigh number should be smaller for Biot numbers that are less than 100 . However, even for $B i=1$, it is shown that the convective cell pattern behaves in a similar manner.

To further examine the effect of the aspect ratio on convection, Figure 8 shows in more detail the streamline and temperature variation pattern for aspect ratios ranging from $A R$ $=1$ to $A R=6$ for the case of $B i=1$. The critical porous layer thickness value is exceeded at $d_{2}=0.97$ and the flow is taking place in the porous regime. It is found that as the aspect ratio increases, multi cellular flow begins to form in the porous cavity. This is most evident when the aspect ratio $A R$ is equal to 6 in Figure $8 \mathrm{~d}$. The strength of the flow also increases with a maximum streamline value of $3.51 \times 10^{-4}$ for $A R=1$ and $6.71 \times 10^{-4}$ for $A R=6$. A similar convection pattern trend has also been observed by Saghir et al. [11], where the flow was studied for a Biot number of 100 . 

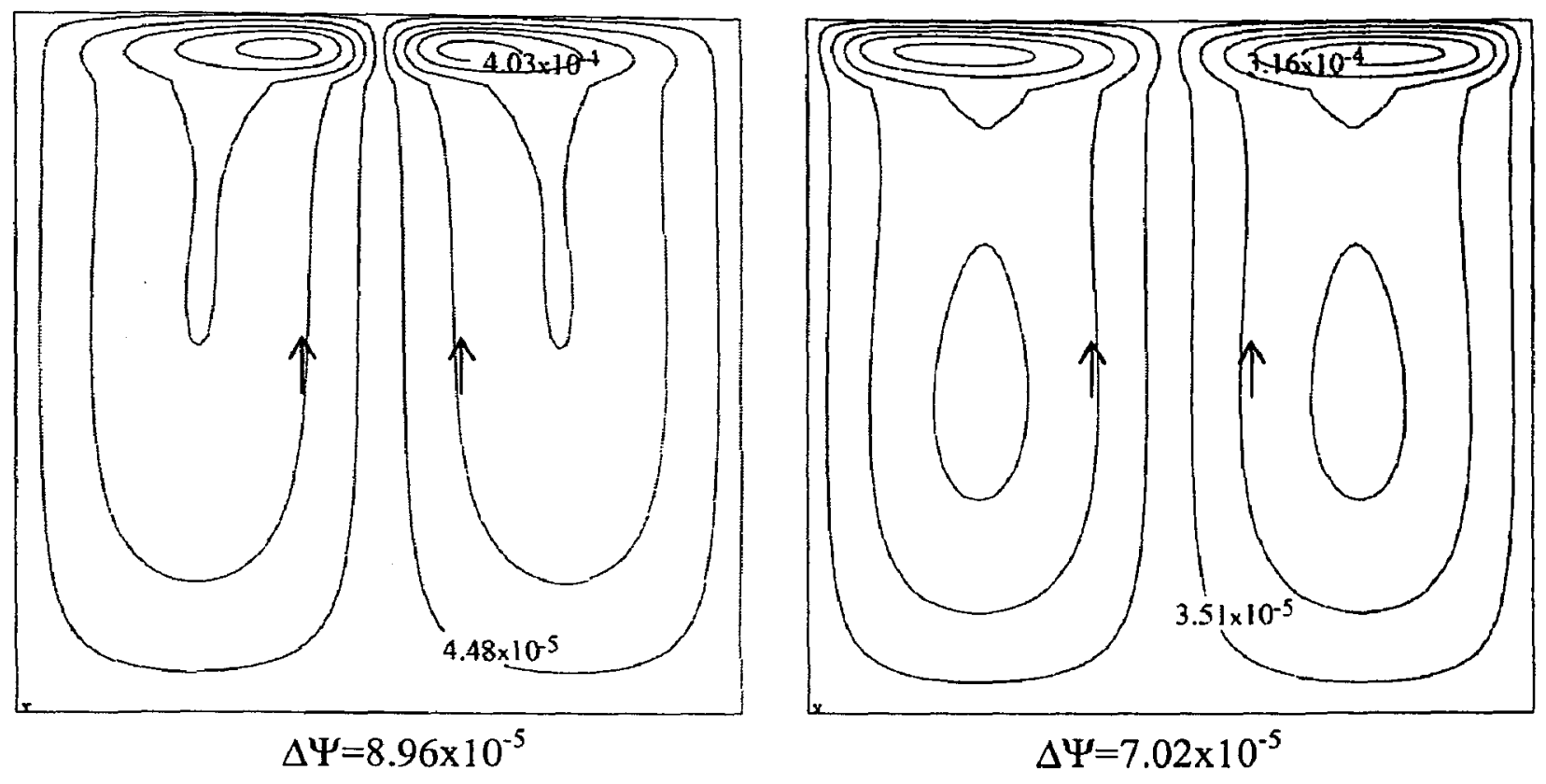

a)
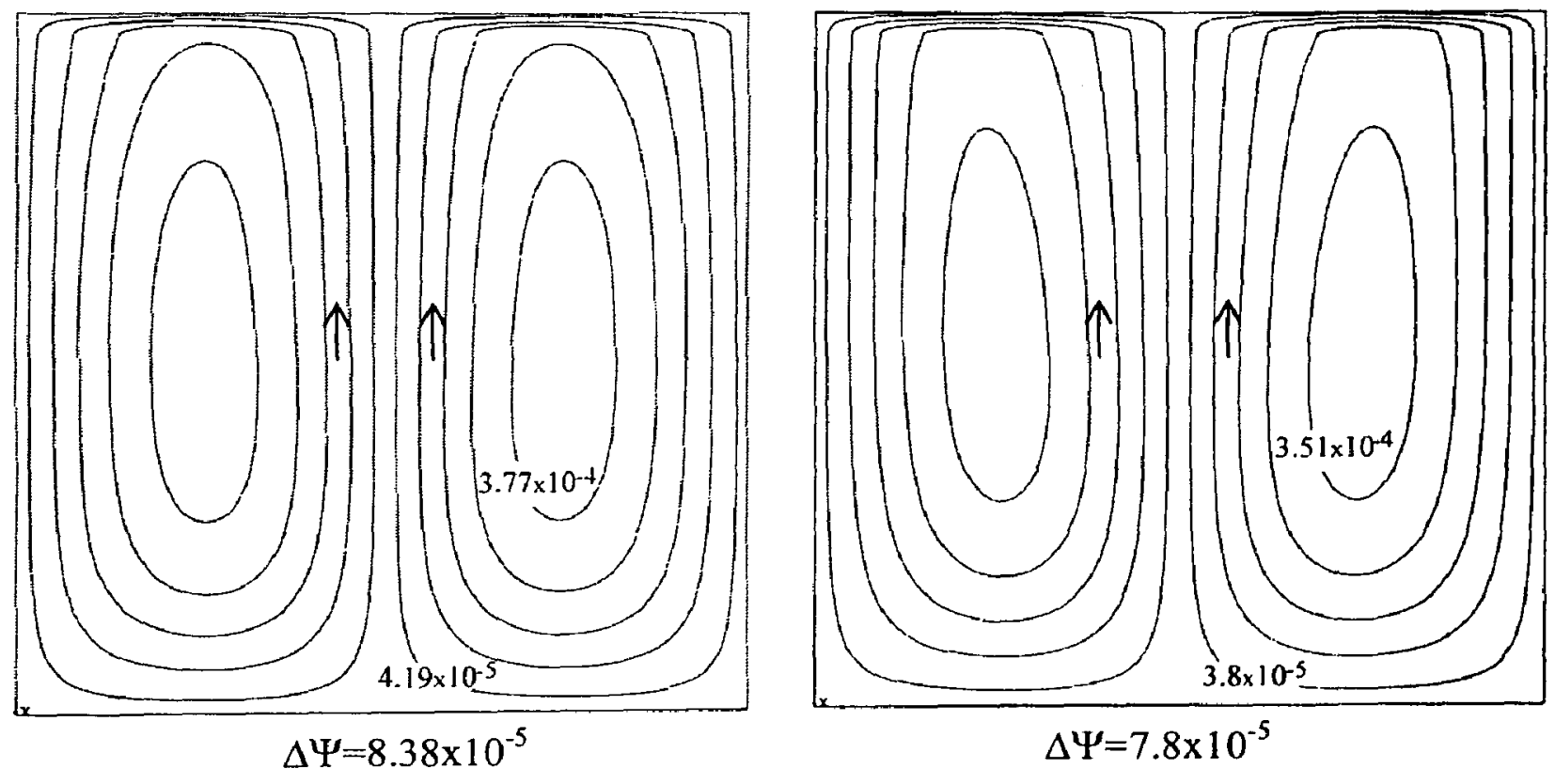

b)

$$
\mathrm{Bi}=100
$$$$
\mathrm{Bi}=1
$$

Figure 7 Buoyancy convection in the cavity for different Biot numbers $(A R=1, \operatorname{Pr}=7)$ :
a) $d_{1}=0.10, d_{2}=0.90, R a_{L}=1000, R a_{P}=55$
b) $d_{1}=0.03, d_{2}=0.97, R a_{L}=100, R a_{P}=75$ 

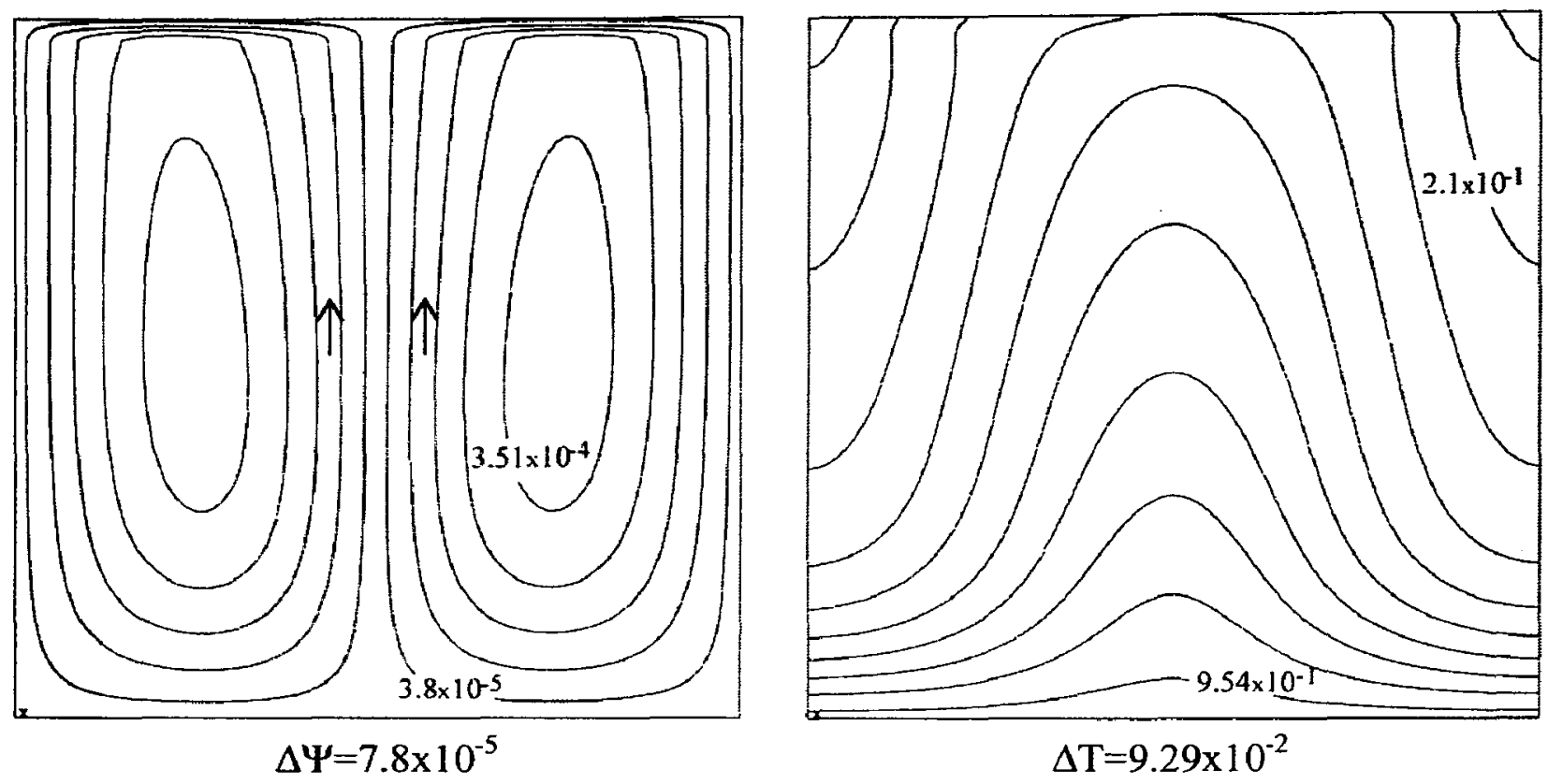

a)
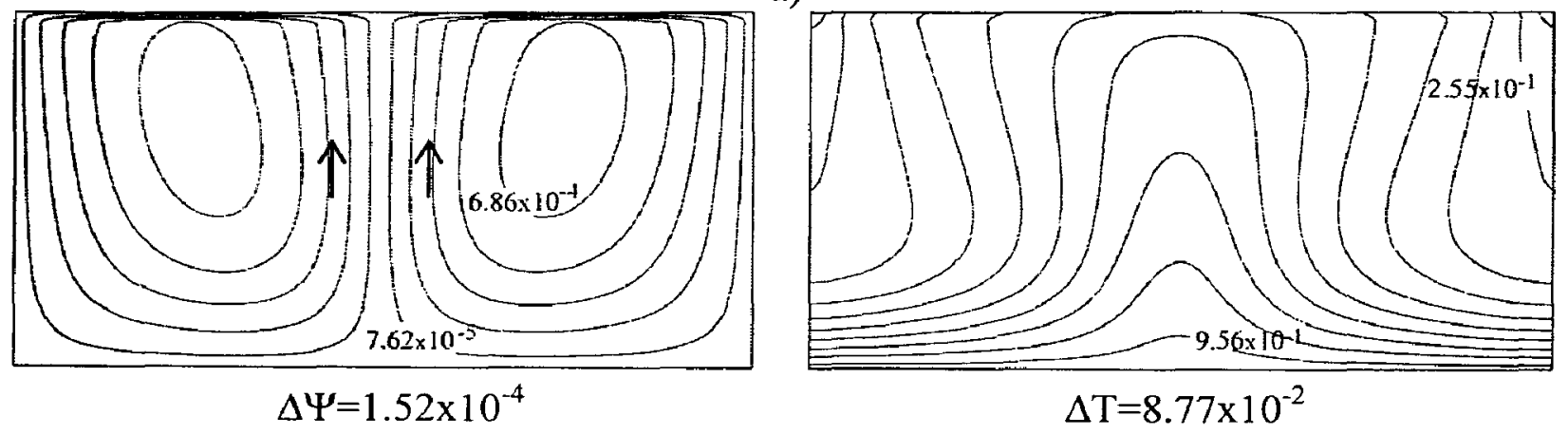

b)
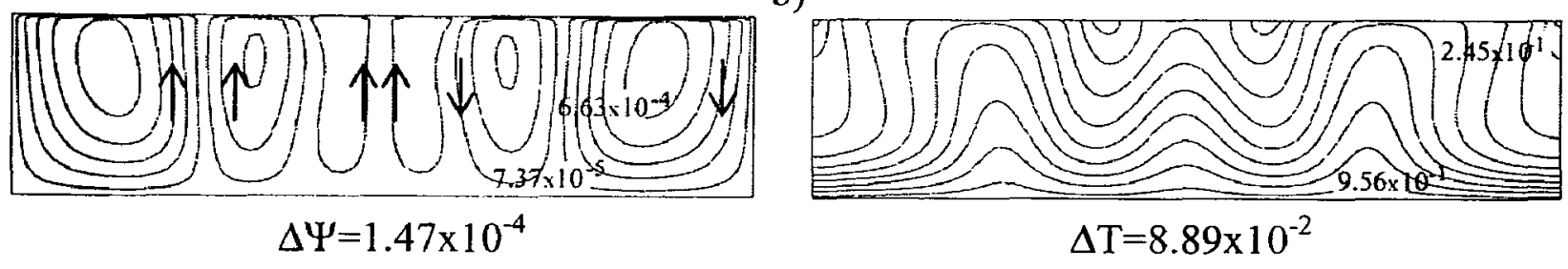

c)
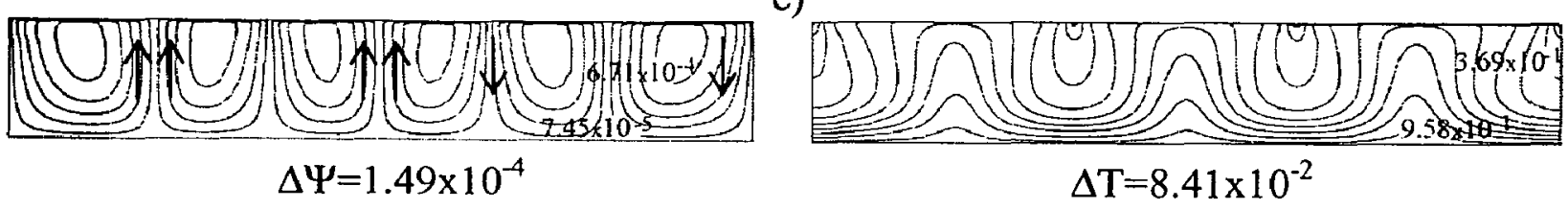

d)

Figure 8 Streamlines and temperature variation in the cavity for different aspect ratios $\left(\mathrm{Ra}_{\mathrm{L}}=100, \mathrm{Ra}_{\mathrm{P}}=75, \mathrm{~d}_{1}=0.03, \mathrm{~d}_{2}=0.97, \mathrm{Bi}=1, \mathrm{Pr}=7\right)$ :
a) $\mathrm{AR}=1$
b) $\mathrm{AR}=2$
c) $\mathrm{AR}=4$
d) $\mathrm{AR}=6$ 


\subsubsection{Bottom Heating Marangoni Convection}

For Marangoni convection in a bottom heated cavity, Desaive et al. [8] found that the critical depth thickness $d_{2}$ was equal to 0.96 . Above this value, the flow dominated the entire porous layer, and below this value, it was limited to the fluid layer. They also found a relationship between $d_{2}$ and the critical Marangoni number. For the case of $d_{2}=$ 0.96 , the critical Marangoni number was found to be 60000 . In Figure 9, the convection pattern is again studied for thermocapillary convection for different aspect ratios and Biot numbers. The flow dominates the porous layer with the porous layer thickness above the critical value of $d_{2}=0.97$. The aspect ratio is once again increased from $A R=1$ to $A R=$ 6 and the Biot varied from $B i=100$ to $B i=1$. In both cases it is shown that the convection pattern differs greatly from the buoyancy flow pattern in the porous layer, as multiple convection cells do not form as the aspect ratio is increased. Therefore, for flow in the porous regime, the aspect ratio does not have any effect on the number of cells. The flow also generally becomes weaker with the increasing aspect ratio but much stronger with decreasing Biot number. However, once again the phenomenon of the counter rotating cells shifting to the lateral walls is noticed when the Biot number is taken to equal 1. It is also shown that a Biot number below 100 does not have an effect on the convection pattern in the cavity. Therefore, a detailed study is performed next on fluid dominated flow with a Biot number equal to 1 .

In Figure 10, thermocapillary convection is presented with $M a=60000$ and $B i=1$. The convection is limited to the liquid layer by setting the porous layer thickness equal to the liquid layer thickness such as $d_{1}=d_{2}=0.5$, which is below the critical thickness value. When the flow is dominated by the liquid layer, multi cell formation is once again observed as the aspect ratio is varied from $A R=1$ to $A R=6$ with multi cells forming accordingly. A similar trend has also been observed by Saghir et al. [11], who found the number of convective cells to be dependant on the available space in the cavity. The strength of the flow also becomes greater with the formation of more convective cells, with the maximum streamline value for $A R=1$ being $1.14 \times 10^{-1}$ and for $A R=6$ the value being $2.79 \times 10^{-1}$. 


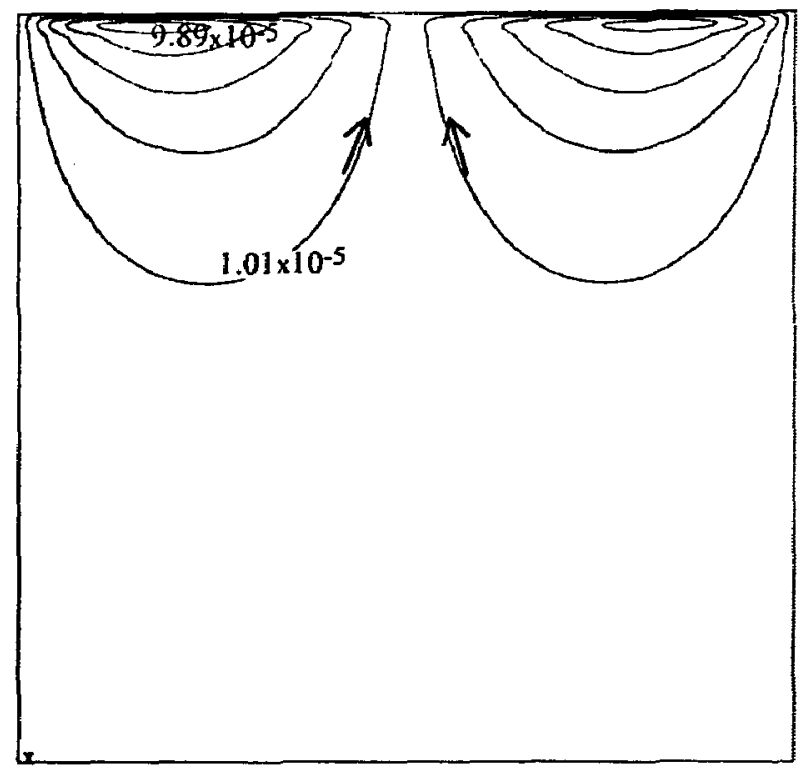

$\Delta \Psi=2.198 \times 10^{-5}$

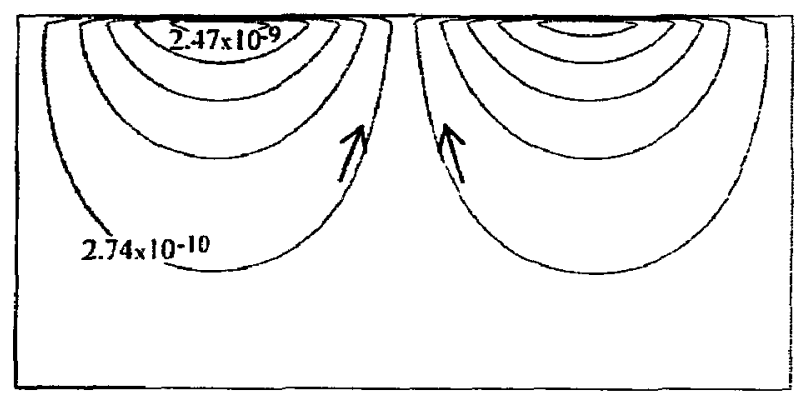

$\Delta \Psi=5.48 \times 10^{-10}$
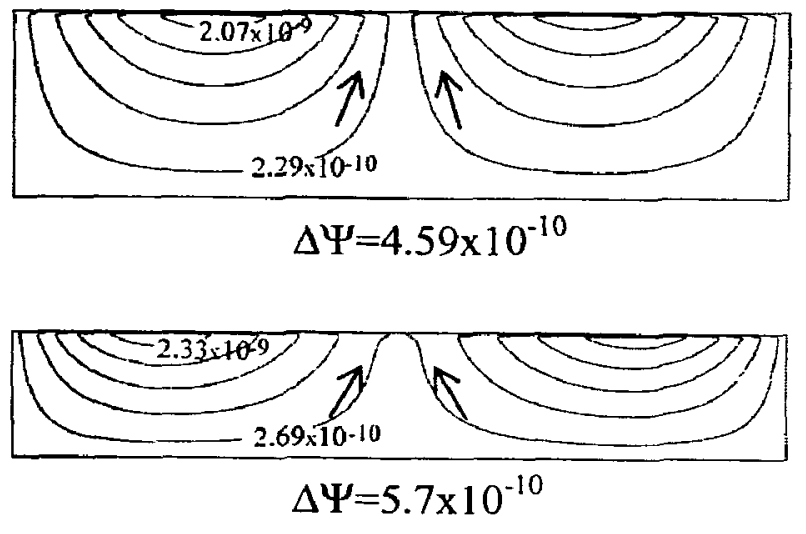

$\Delta \Psi=5.7 \times 10^{-10}$

$\mathrm{Bi}=100$

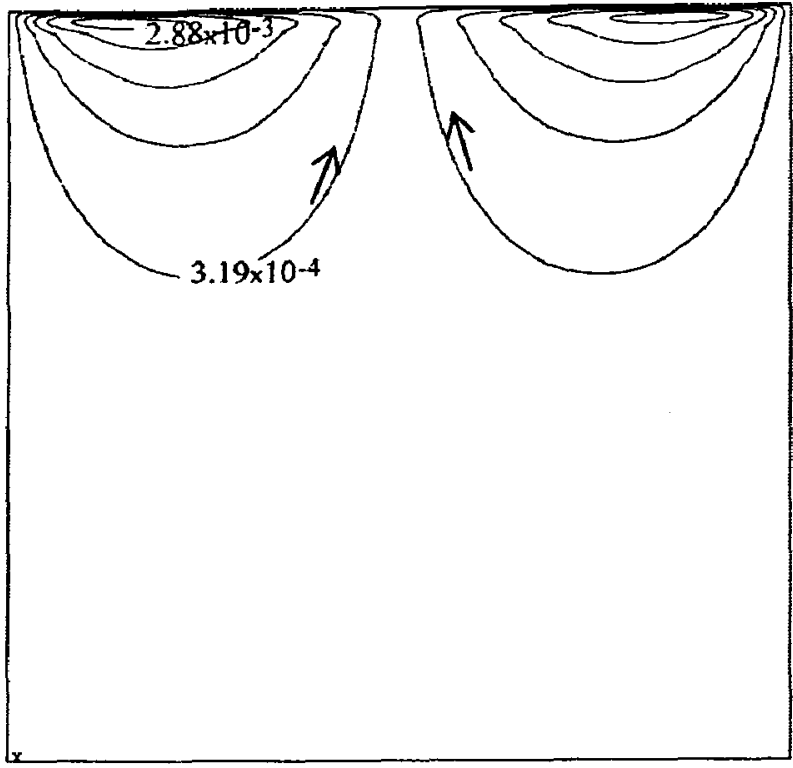

$\Delta \Psi=6.39 \times 10^{-4}$

a)

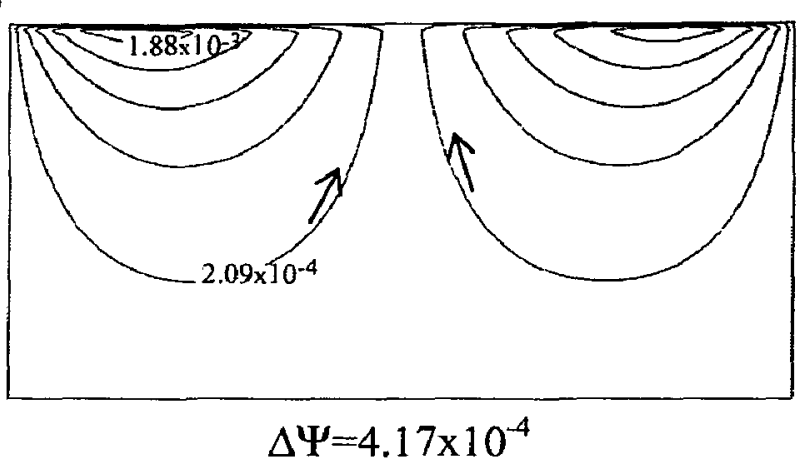

b)

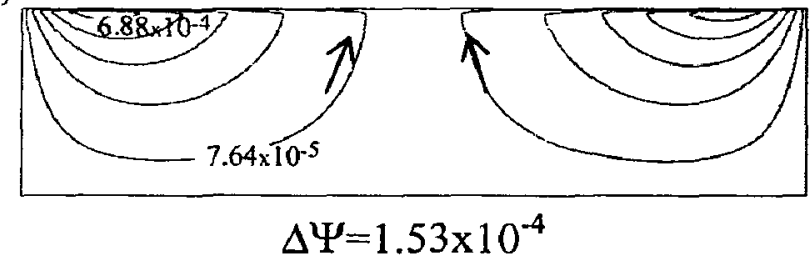

c)

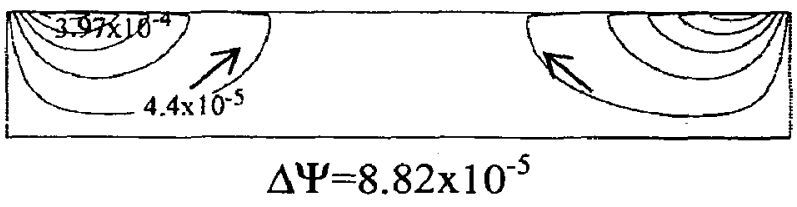

d)

$\mathrm{Bi}=1$

Figure 9 Thermocapillary convection in the cavity for different aspect ratios and Biot numbers $\left(\mathrm{Ma}=60000, \mathrm{Ra}_{\mathrm{L}}=0.1, \mathrm{Ra}=0.075, \mathrm{~d}_{1}=0.03, \mathrm{~d}_{2}=0.97, \operatorname{Pr}=7\right)$ :
a) $\mathrm{AR}=1$
b) $\mathrm{AR}=2$
c) $\mathrm{AR}=4$
d) $\mathrm{AR}=6$ 

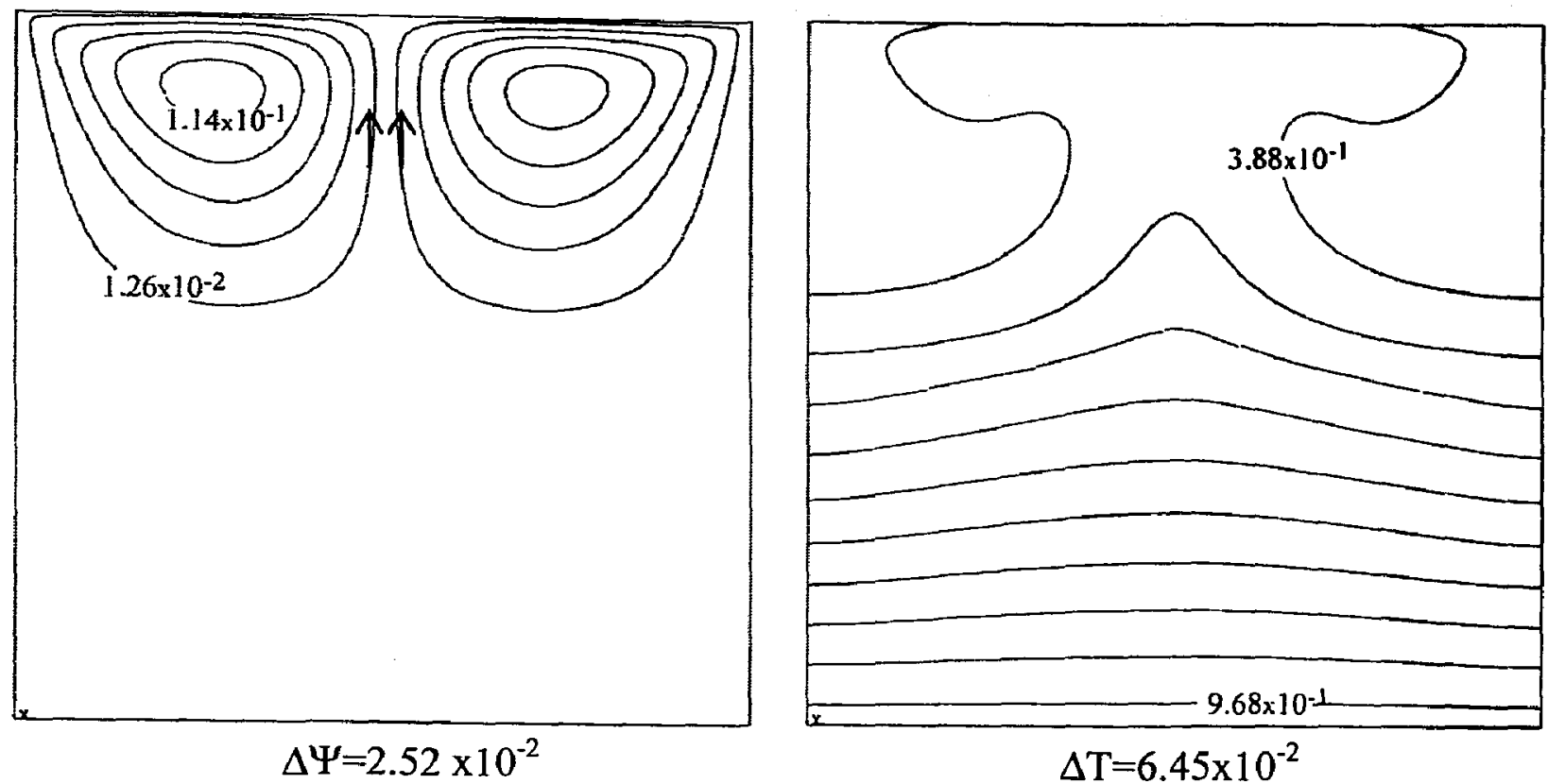

a)
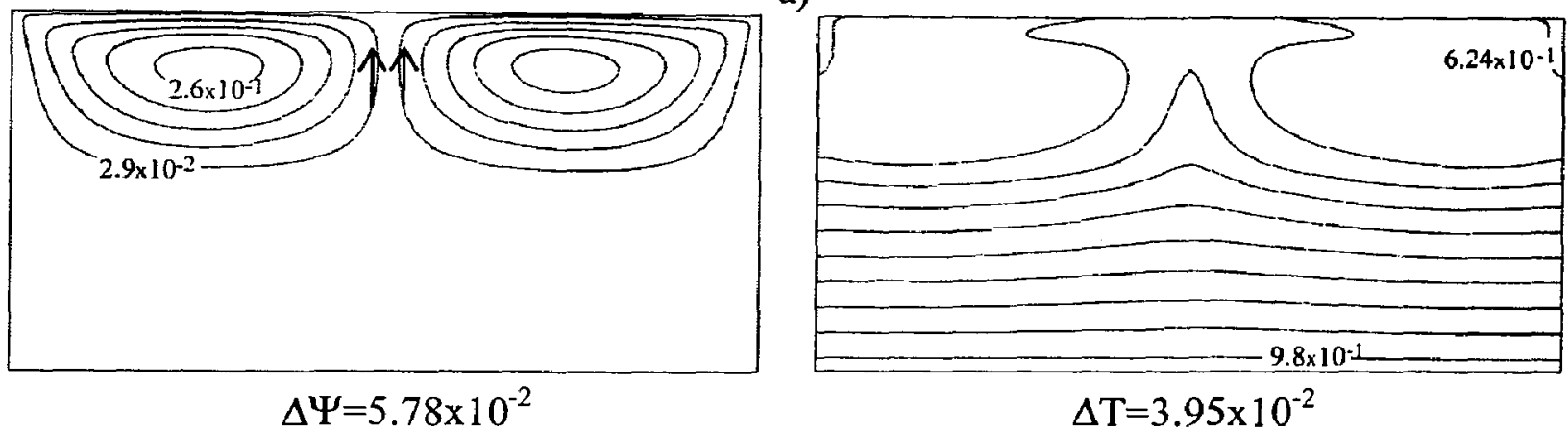

b)

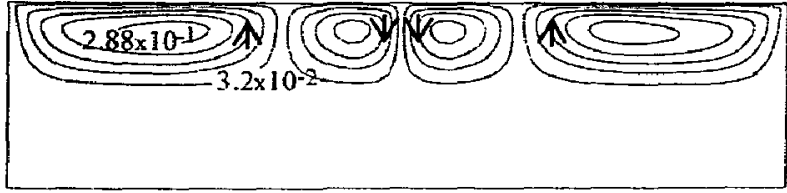

$\Delta \Psi=6.41 \times 10^{-2}$

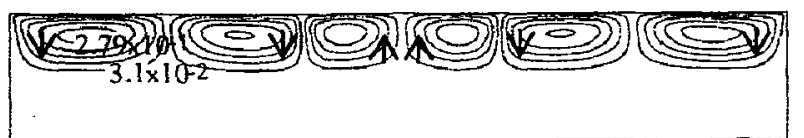

$\Delta \Psi=6.21 \times 10^{-2}$

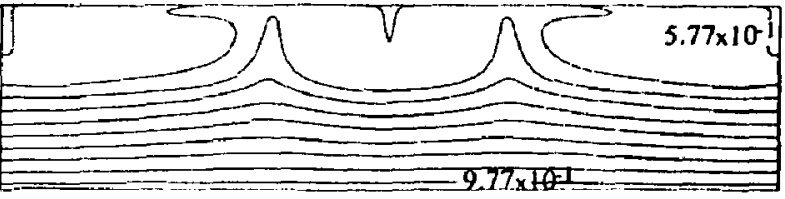

$$
\Delta \mathrm{T}=4.45 \times 10^{-2}
$$

c)

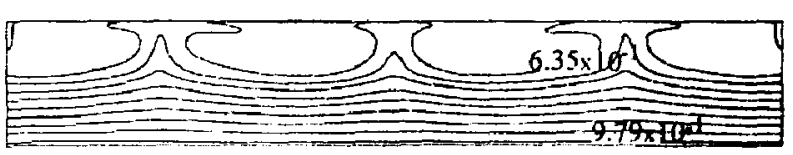

$$
\Delta \mathrm{T}=4.3 \times 10^{-2}
$$

d)

Figure 10 Streamlines and temperature variation in the cavity for different aspect ratios

$$
\left(\mathrm{Ma}=60000, \mathrm{Ra}_{\mathrm{L}}=0.1, \mathrm{Ra}_{\mathrm{P}}=0.0055, \mathrm{~d}_{1}=\mathrm{d}_{2}=0.5, \mathrm{Bi}=1, \operatorname{Pr}=7\right) \text { : }
$$
a) $\mathrm{AR}=1$
b) $A R=2$
c) $A R=4$
d) $\mathrm{AR}=6$ 


\subsubsection{Lateral Heating Buoyancy Convection}

The lateral heating condition is also closely examined, as it is well known that the applied heat transfer insures motion. First buoyancy convection is studied with varied porous layer thicknesses. In the following sections, thermocapillary and combined convection is also studied for the lateral heating condition.

Figure 11 shows buoyancy convection for different aspect ratios with the liquid Rayleigh number held constant at $R a_{L}=1000$. When the porous layer thickness is set to $d_{2}=0.5$, the flow remains in the liquid layer as the aspect ratio is increased from $A R=2$ to $A R=4$. When $d_{2}=0.97$, the flow similarly dominates the porous layer in the cavity with the cell now within the porous layer itself. It is also noted that for the case of $d_{2}=0.97$, as the aspect ratio increases the flow gets stronger. The maximum streamline value increases from $8.59 \times 10^{-4}$ for $A R=2$ to $8.84 \times 10^{-4}$ for $A R=4$.

Figure 12 investigates buoyancy convection as the liquid Rayleigh number is decreased from 1000 to 400 for the case when the porous layer thickness is set to $d_{2}=0.5$ and $d_{2}=$ 0.97 . When the flow dominates the liquid layer, it can be seen that no significant changes occur to the streamline convection pattern as the Rayleigh number decreases. However, the flow weakens considerably, with the streamline value decreasing from $6.08 \times 10^{-3}$ to $4.04 \times 10^{-3}$ for the liquid Rayleigh number equal to 1000 and 400 respectively. As the flow dominates the porous layer with $d_{2}=0.97$, it is noticed that the center of the convective cell does not penetrate as deep into the cavity with the Rayleigh number decreased to $R a_{L}=400$. However, there is little difference in the streamline values between the two cases and the flow is still dominant in the porous layer. This has also been observed by Saghir et al. [11], when studying smaller values of the Rayleigh number. Therefore, the Rayleigh number has more of an effect on the strength of the flow, when the flow is constrained to the liquid layer, as opposed to the porous layer. 


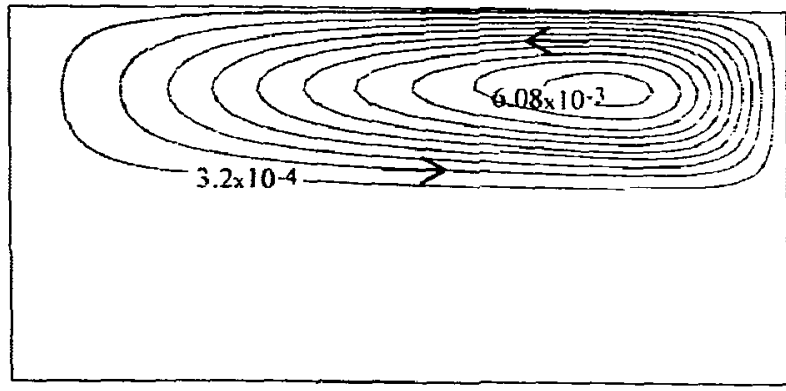

$\Delta \Psi=6.4 \times 10^{-4}$

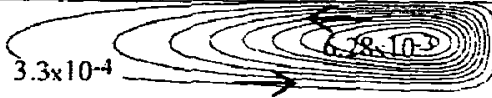

$\Delta \Psi=6.61 \times 10^{-4}$

$\mathrm{d}_{1}=\mathrm{d}_{2}=0.5$

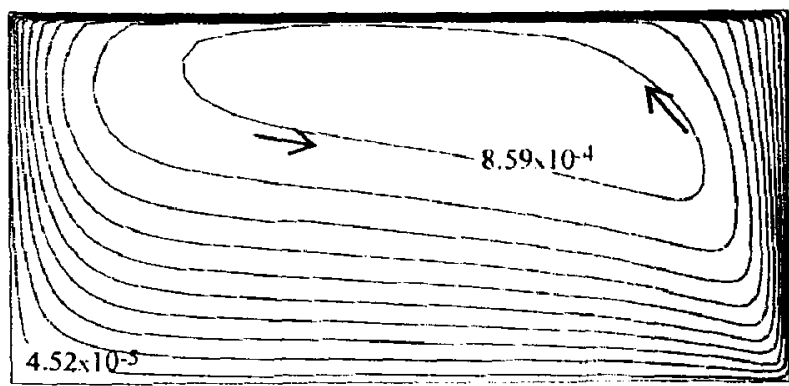

$\Delta \Psi=9.04 \times 10^{-5}$

a)

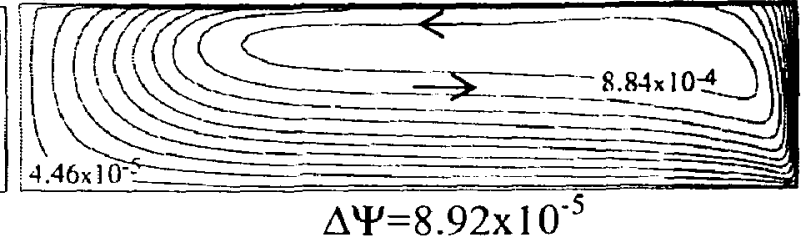

b)

$\mathrm{d}_{1}=0.03 \mathrm{~d}_{2}=0.97$

Figure 11 Buoyancy convection in the cavity for different aspect ratios

$$
\left(\mathrm{Ra}_{\mathrm{L}}=1000, \mathrm{Ma}=1, \mathrm{Bi}=1, \mathrm{Pr}=7\right): \text { a) } \mathrm{AR}=2 \text { b) } \mathrm{AR}=4
$$

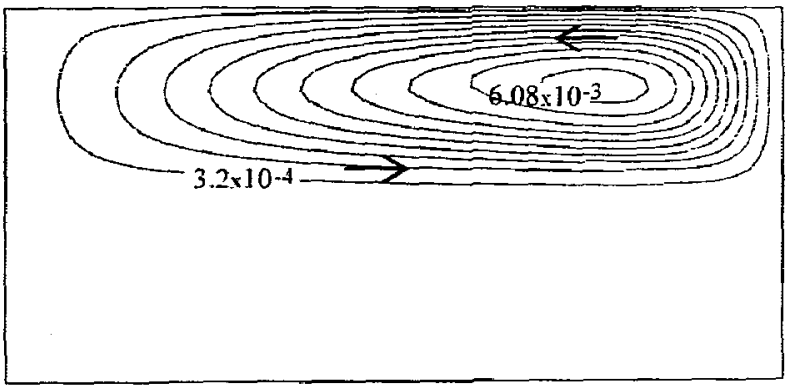

$\Delta \Psi=6.4 \times 10^{-4}$

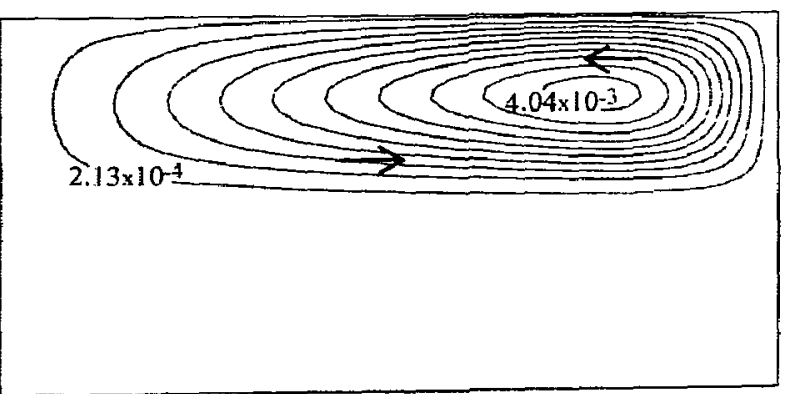

$\Delta \Psi=4.25 \times 10^{-4}$

$\mathrm{d}_{1}=\mathrm{d}_{2}=0.5$

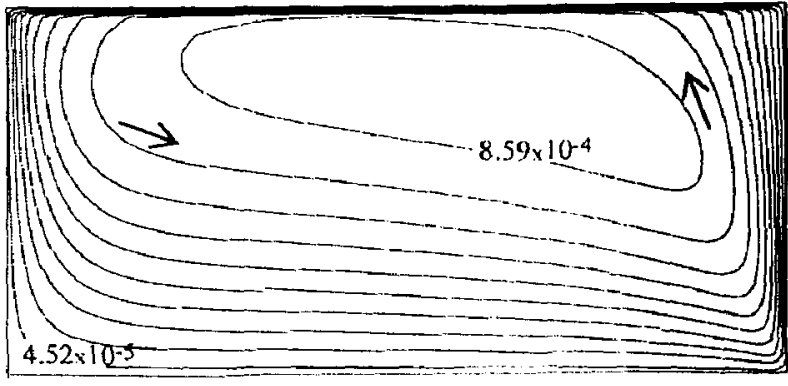

$\Delta \Psi=9.04 \times 10^{-5}$

a)

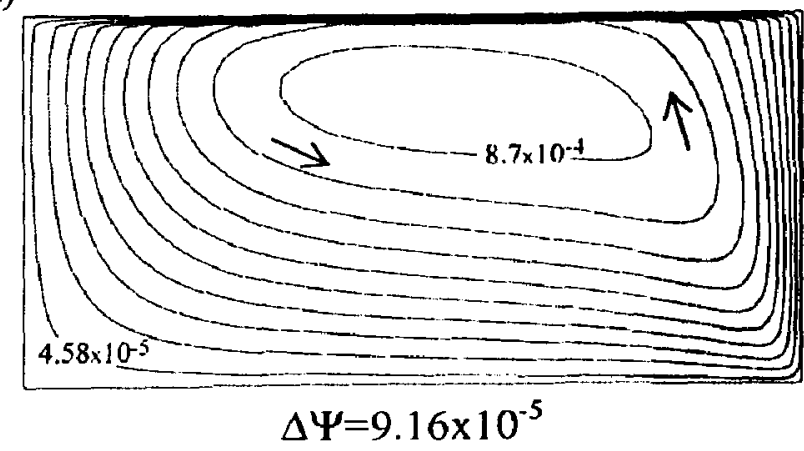

b)

$$
\mathrm{d}_{1}=0.03 \mathrm{~d}_{2}=0.97
$$

Figure 12 Buoyancy convection in the cavity

$$
\left(\mathrm{Ma}=1, \mathrm{AR}=2, \mathrm{Bi}=1, \mathrm{Pr}=7 \text { ): a) } \mathrm{Ra}_{\mathrm{L}}=1000 \text { b) } \mathrm{Ra}_{\mathrm{L}}=400\right.
$$




\subsubsection{Lateral Heating Marangoni Convection}

Several cases are studied in detail for a liquid layer overlying a porous layer in a laterally heated cavity for thermocapillary convection. The Marangoni number is kept constant and equal to $M a=60000$, and the liquid Rayleigh number is kept constant and equal to $R a_{L}=1$ during the entire analysis. It should be noted that the liquid Rayleigh number is not zero but is very small in comparison to the Marangoni number. This approach ensures that Marangoni convection will be the dominant force. To mimic a microgravity situation, the gravity vector is assumed to be equal to $10^{-4} \mathrm{~g}$.

In the first case, the flow structure is examined as it varies with the aspect ratio and is compared to a similar analysis by Saghir et al. [11]. In the second case, thermocapillary convection is examined for different Marangoni numbers and thicknesses of liquid and porous layers. Again a comparison is made to the results obtained by Saghir et al. [11].

Thermocapillary convection is presented for aspect ratios of $A R=2$ and $A R=4$ in Figure 13. For a porous layer thickness of $d_{2}=0.5$, the flow is limited to the liquid layer. The porous layer acts as a solid wall and the flow remains entirely in the liquid layer. When the aspect ratio is equal to 2 , there is a large convective cell with the center near the middle of the cavity present in the liquid layer. The convection is said to be monocellular. However, as the aspect ratio is increased to 4 , multi-cellular flow becomes apparent. This has also been predicted by Villers and Platten [10] and their result of increasing the aspect ratio, is shown in Figure 13c. Therefore, in a finite cavity, the number of cells depends on the available space, in essence the aspect ratio.

As the thickness of the liquid layer is minimized and the porous layer is set to $d_{2}=0.97$, the flow becomes dominant in the porous layer. It is noticed that a large convective cell invades the porous cavity for both values of the aspect ratio, with the center of the cell close to the free surface and liquid layer. However, the aspect ratio has no effect on the number of convective cells present in the porous layer. The streamlines get weaker as the aspect ratio increases, which results in reduction of size of the convective cell. The flow 
also gets weaker when the convection is switched from liquid layer dominated to porous layer dominated, due to the smaller reach of Marangoni convection within the porous layer.

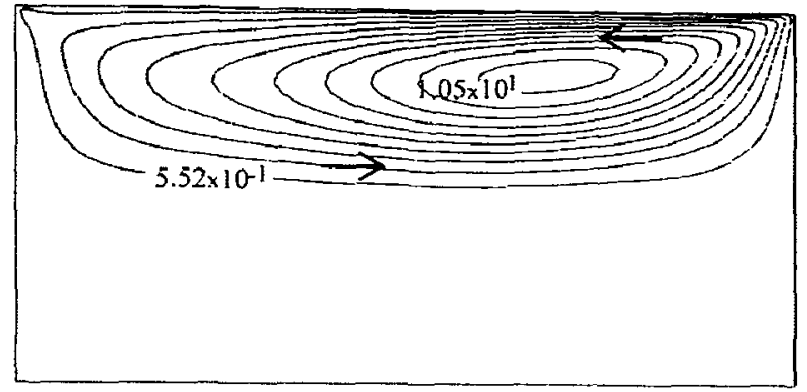

$\Delta \Psi=1.105$

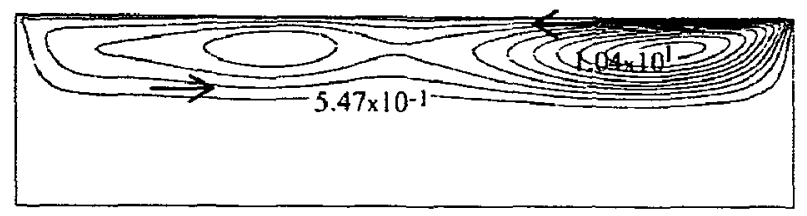

$\Delta \Psi=1.094$

$\mathrm{d}_{1}=\mathrm{d}_{2}=0.5$

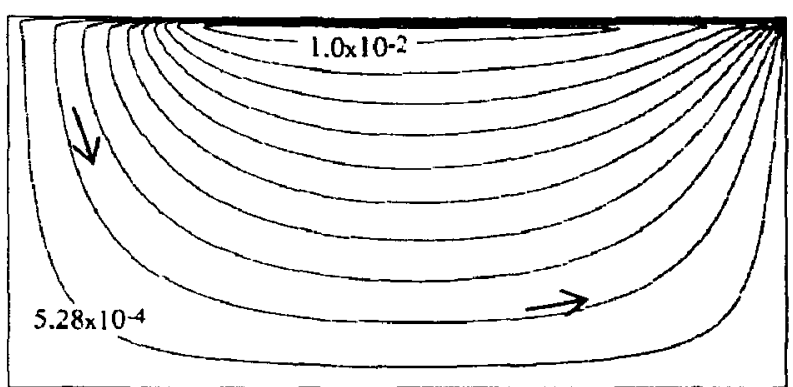

$\Delta \Psi=1.06 \times 10^{-3}$

a)

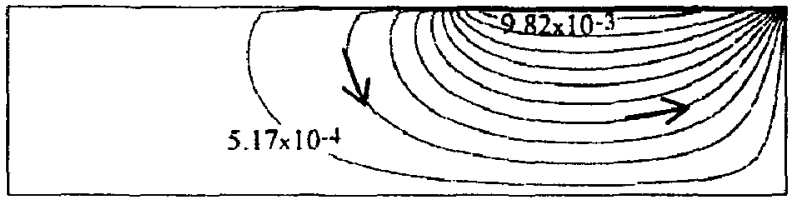

$\Delta \Psi=1.033 \times 10^{-3}$

b)

$\mathrm{d}_{1}=0.03 \mathrm{~d}_{2}=0.97$

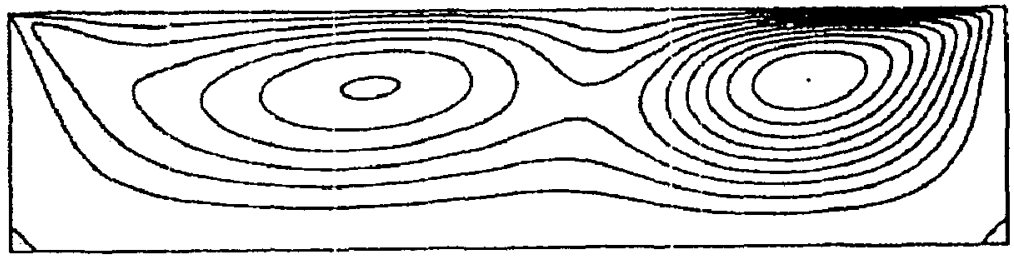

c)

Figure 13 Thermocapillary convection in the cavity for different aspect ratios ( $\mathrm{Ma}=60000, \mathrm{Ra}_{\mathrm{L}}=1, \mathrm{Bi}=1, \mathrm{Pr}=7$ ):
a) $\mathrm{AR}=2$
b) $\mathrm{AR}=4$
c) $\mathrm{AR}=4, \mathrm{Ma}=8000, \mathrm{Pr}=4$ Villers and Platten [10]

The effect of varying the Marangoni number is illustrated in Figure 14, with the aspect ratio kept constant at $A R=2$ and the flow dominant in the liquid layer. As the Marangoni number was decreased from 60000 to 30000 and finally to 10000 , the flow got considerably weaker, with the maximum streamline value equal to $1.05 \times 10^{1}, 5.93$ and 
1.88 respectively. It is also noticed that the center of the convective cell shifted towards the hot side wall as the Marangoni number got smaller and the flow weaker. This trend enforces the fact that a laterally heated cavity ensures fluid motion, without dependence on a critical Marangoni number.

Figure 15 depicts thermocapillary convection in the cavity for aspect ratios of $A R=2$ and $A R=4$ with $d_{2}=0.5$ being held constant. The flow remains dominant in the liquid layer, with multi cellular flow still forming at $M a=30000$. However, as the Marangoni number is decreased to $M a=10000$, multi cellular formation is no longer observed in the convection pattern. The flow also gets stronger as the aspect ratio is increased with a maximum streamline value of 5.93 for $A R=2$ and 6.98 for $A R=4$ in the case of $M a=$ 30000. In the case of $M a=10000$, the streamline value increases from 1.88 for $A R=2$ to 2.41 for $A R=4$.

The porous layer thickness is also varied from $d_{2}=0.5$ to $d_{2}=0.97$ to study the convection pattern when $M a=30000$ and $M a=10000$ in Figure 16. As the porous layer thickness is increased, the flow switches from liquid layer dominant flow to porous layer dominant flow. When $M a=30000$ and $d_{2}=0.97$, the flow penetrates to the bottom of the porous cavity. However, when the Marangoni number is decreased further, the flow no longer penetrates right to the bottom of the cavity due to the weaker flow. The maximum streamline value decreases from $7.11 \times 10^{-3}$ to $2.85 \times 10^{-3}$. 


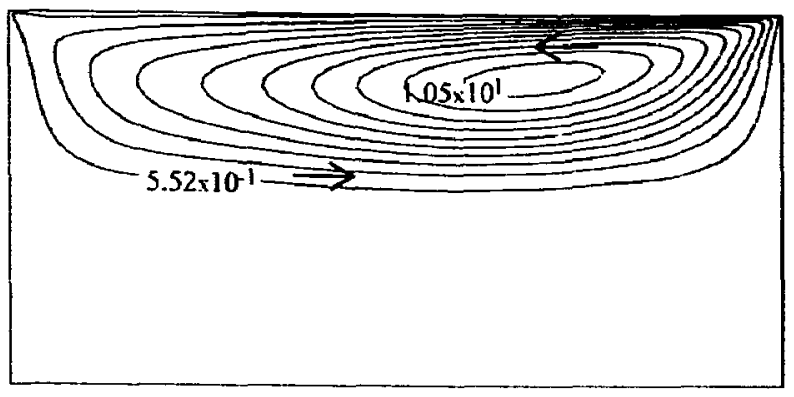

a) $\Delta \Psi=1.105$

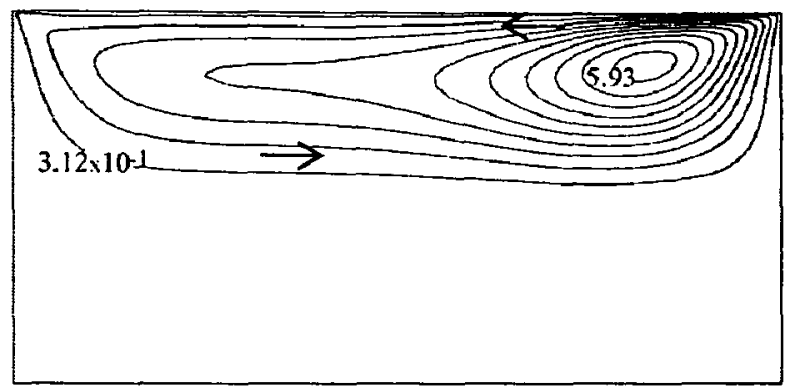

b) $\Delta \Psi=6.25 \times 10^{-1}$

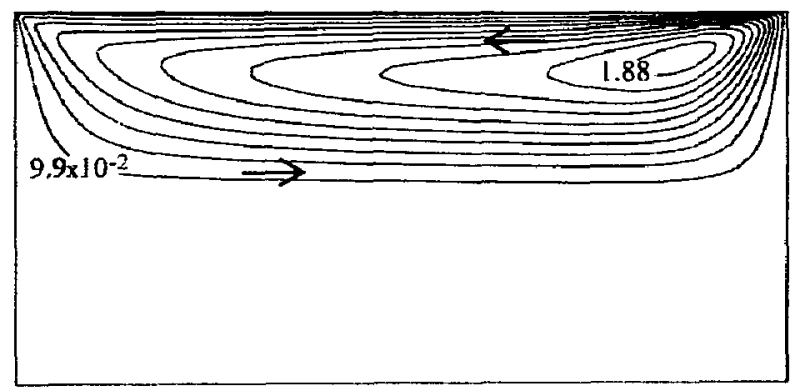

c) $\Delta \Psi=1.98 \times 10^{-1}$

Figure 14 Thermocapillary convection in the cavity for different Marangoni numbers $\left(\mathrm{Ra}_{\mathrm{L}}=1, \mathrm{~d}_{1}=\mathrm{d}_{2}=0.5, \mathrm{AR}=2, \mathrm{Bi}=1, \mathrm{Pr}=7\right)$ :
a) $\mathrm{Ma}=60000$
b) $M a=30000$
c) $\mathrm{Ma}=10000$ 


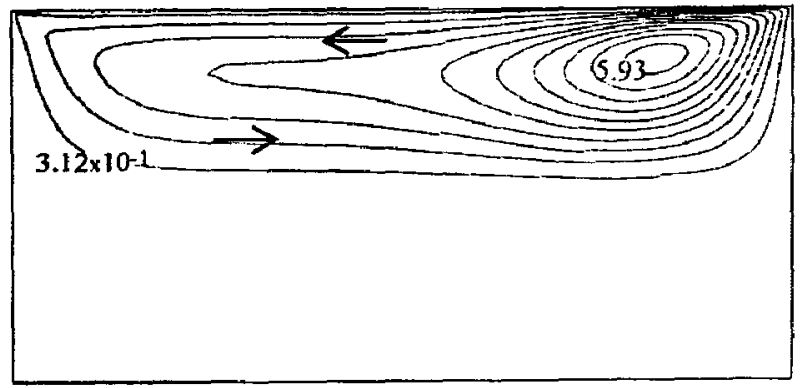

$\Delta \Psi=6.25 \times 10^{-1}$

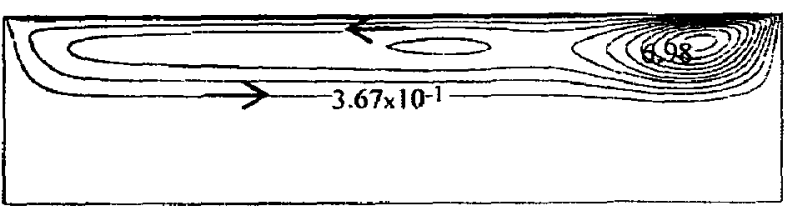

$\Delta \Psi=7.34 \times 10^{-1}$

$\mathrm{Ma}=30000$

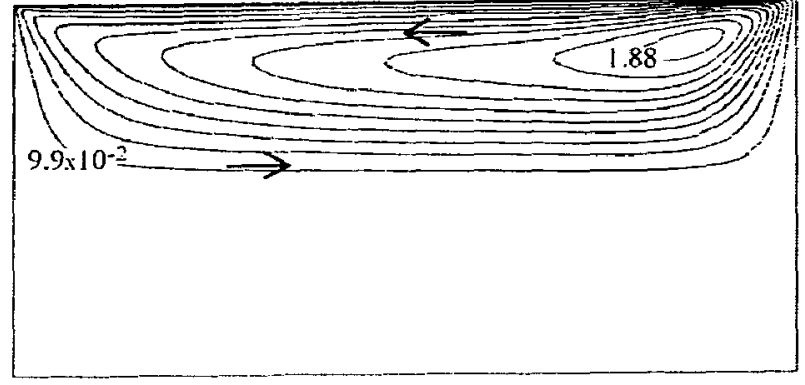

$\Delta \Psi=1.98 \times 10^{-1}$

a)

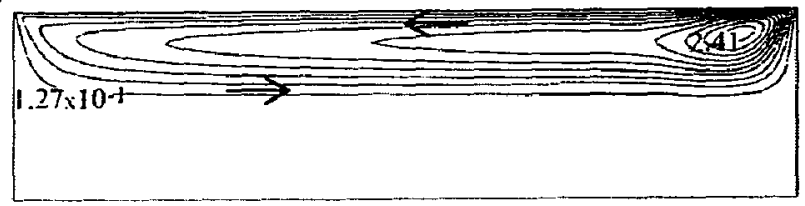

$\Delta \Psi=2.54 \times 10^{-1}$

b)

$\mathrm{Ma}=10000$

Figure 15 Thermocapillary convection in the cavity for different aspect ratios

$$
\left(\mathrm{Ra}_{\mathrm{L}}=1, \mathrm{~d}_{1}=\mathrm{d}_{2}=0.5, \mathrm{Bi}=1, \operatorname{Pr}=7\right) \text { : }
$$
a) $\mathrm{AR}=2$
b) AR-4

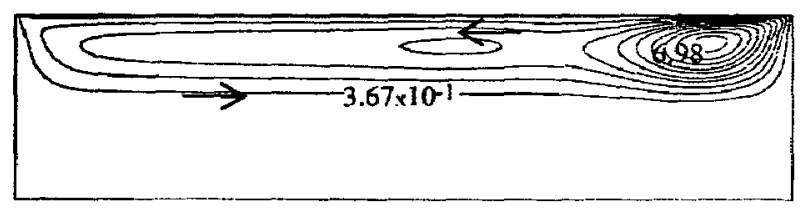

$\Delta \Psi=7.34 \times 10^{-1}$

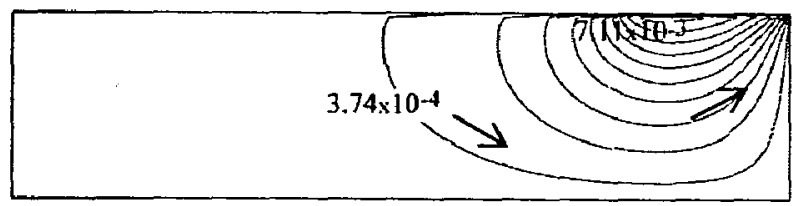

$\Delta \Psi=7.48 \times 10^{-4}$

$\mathrm{Ma}=30000$

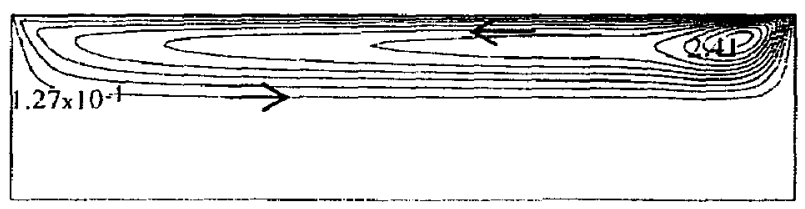

$\Delta \Psi=2.54 \times 10^{-1}$

a)

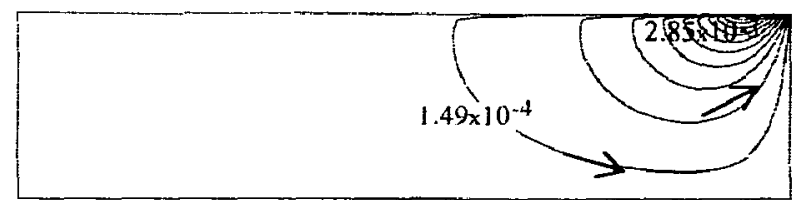

$\Delta \Psi=3.0 \times 10^{-4}$

b)

$\mathrm{Ma}=10000$

Figure 16 Thermocapillary convection in the cavity for different thickness $d_{1}$ and $d_{2}$

$$
\left(\mathrm{Ra}_{\mathrm{L}}=1, \mathrm{AR}=4, \mathrm{Bi}=1, \mathrm{Pr}=7\right) \text { : }
$$
a) $\mathrm{d}_{1}=\mathrm{d}_{2}=0.5$
b) $d_{1}=0.03, d_{2}=0.97$ 


\subsubsection{Lateral Heating Combined Buoyancy and Marangoni Convection}

To study combined buoyancy and Marangoni convection, Figure 17 shows the case where the Marangoni number is equal to 60000 and the liquid Rayleigh number is equal to 1000 . When the flow is limited to the liquid layer the streamline pattern is very similar to that of Marangoni convection alone, with multi cellular convection also forming as the aspect ratio is increased. When compared to the case of buoyancy convection alone in Figure $11 \mathrm{a}$, it can be seen that Marangoni convection significantly enhances the flow in the liquid layer with the maximum streamline value increasing from $6.08 \times 10^{-3}$ to $3.32 \times 10^{-1}$. However, when the flow penetrates into the porous layer, the flow is mainly due to the buoyancy convection with the streamline patterns being similar to that of buoyancy convection alone.

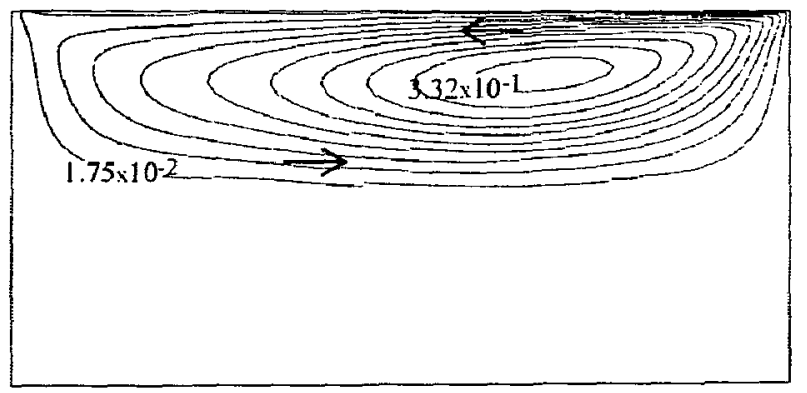

$\Delta \Psi=3.5 \times 10^{-2}$

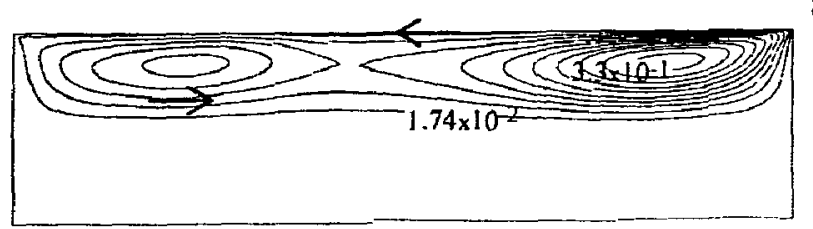

$\Delta \Psi=3.48 \times 10^{-2}$

$\mathrm{d}_{1}=\mathrm{d}_{2}=0.5$

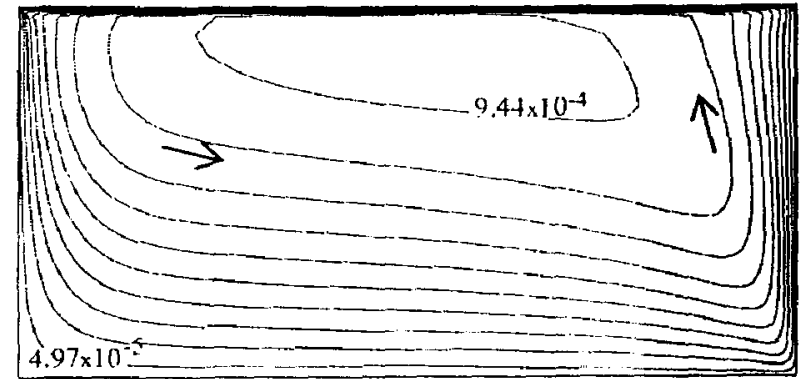

$\Delta \Psi=9.94 \times 10^{-5}$

a)

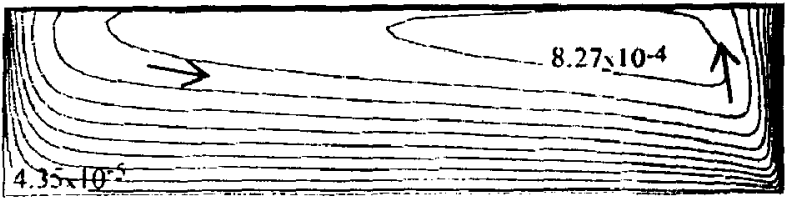

$\Delta \Psi=8.7 \times 10^{-5}$

b)

$$
\mathrm{d}_{1}=0.03 \mathrm{~d}_{2}=0.97
$$

Figure 17 Combined thermocapillary and buoyancy convection in the cavity

$$
\left(\mathrm{Ma}=60000, \mathrm{Ra}_{\mathrm{L}}=1000, \mathrm{Bi}=1, \mathrm{Pr}=7\right) \text { : }
$$
a) $\mathrm{AR}=2$
b) $\mathrm{AR}=4$ 
With the combined buoyancy and Marangoni convection, the center of the convective cell shifts towards the liquid layer and the flow penetrates less into the porous layer. When the aspect ratio is increased, the cell also shifts closer to the hot vertical wall on the right. The Marangoni convection enhances the heat transfer through the free surface leading to the reduced convection in the porous layer. This analysis is comparable to that shown by Saghir et al. [11], where the general flow profiles remained approximately the same for the combined flow case.

Figure 18 shows a similar case to that of Figure 17 but with a decreased Marangoni number of $M a=30000$. The streamline patters are again similar to the case of Marangoni convection alone, when the flow is dominant in the liquid layer but with a weaker flow due to the reduced Marangoni number.

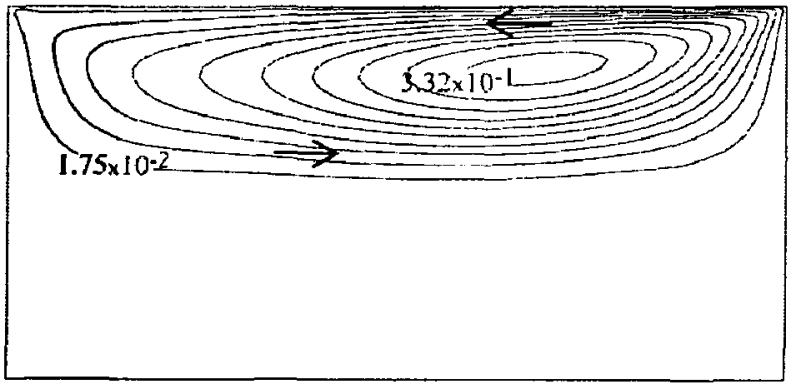

$\Delta \Psi=3.5 \times 10^{-2}$

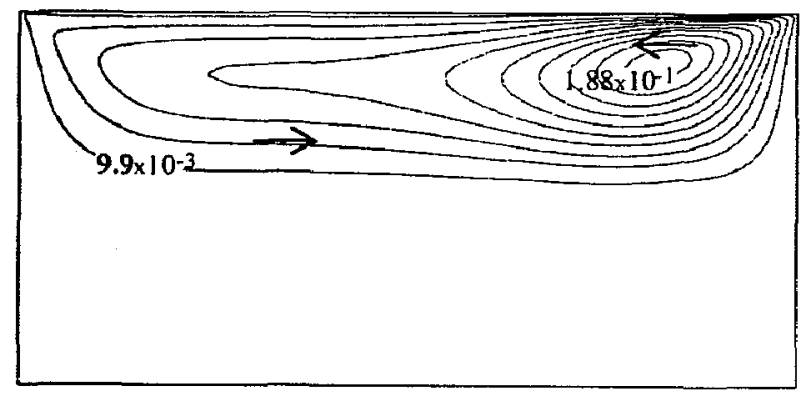

$\Delta \Psi=1.99 \times 10^{-2}$

$\mathrm{d}_{1}=\mathrm{d}_{2}=0.5$

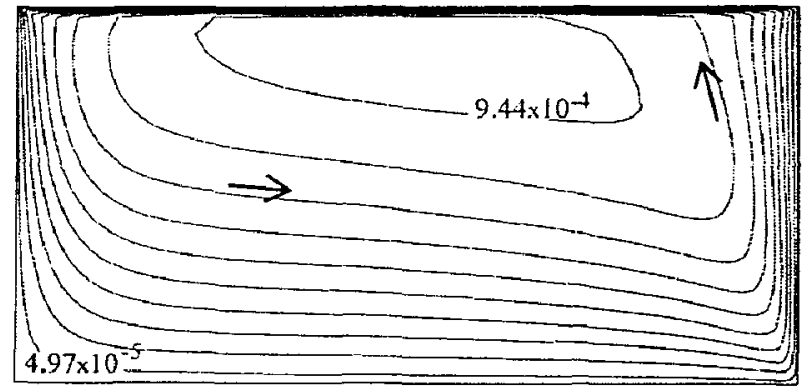

$\Delta \Psi=9.94 \times 10^{-5}$

a)

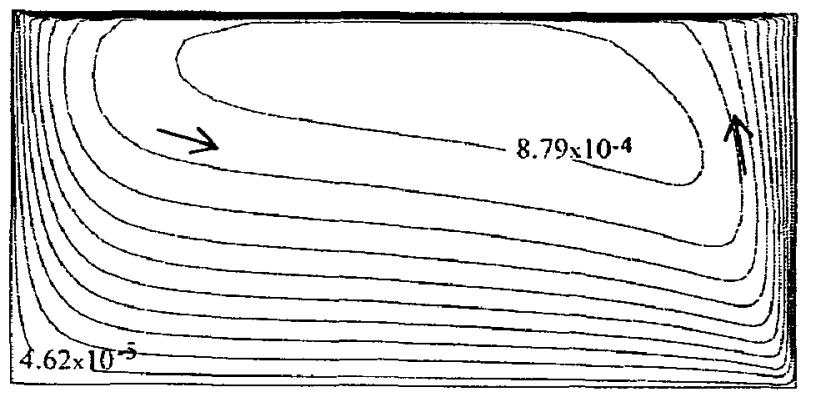

$\Delta \Psi=9.25 \times 10^{-5}$

b)

$$
\mathrm{d}_{1}=0.03 \mathrm{~d}_{2}=0.97
$$

Figure 18 Combined thermocapillary and buoyancy convection in the cavity $\left(\mathrm{Ra}_{\mathrm{L}}=1000, \mathrm{AR}=2, \mathrm{Bi}=1, \mathrm{Pr}=7\right)$ :
a) $\mathrm{Ma}=60000$
b) $\mathrm{Ma}=30000$ 


\subsection{Three Dimensional Modeling}

The results for the three-dimensional modeling are presented as velocity vector plots with a velocity vector drawn at each nodal point. The velocity vector's length is proportional to the fluid speed and the direction of flow is indicated by its inclination. The cavity has a height of $\mathrm{L}$ in the $\mathrm{Y}$-axis and a width of $\mathrm{H}$ in the $\mathrm{X}$-axis, as in the two-dimensional model. A depth of 0.5 is added in the positive $Z$-axis direction to make the cavity threedimensional. To display the results, the cavity is cut right in the middle at 0.25 along the non-dimensional Z-axis.

The three-dimensional modeling section is broken down into subsections that include lateral and bottom heating. Again, different convection types are studied in detail to verify that the two-dimensional model reasonably predicts the three-dimensional results.

\subsubsection{Lateral Heating}

The two-dimensional buoyancy case with liquid Rayleigh number equal to 1000 and a negligible Marangoni number, to prevent thermocapillary convection, has been chosen as a comparison for the three-dimensional model. The porosity is unchanged at 0.39 , with the Darcy number kept at $\mathrm{Da}=10^{-5}$. A case with the flow limited to the fluid layer with $d_{1}=d_{2}=0.5$ is studied with an aspect ratio $A R=4$.

The velocity vector diagram is displayed in Figure 19. It is clearly seen that a similar flow pattern to that of Figure $11 \mathrm{~b}$ has developed in the liquid layer. The characteristic buoyancy type of symmetrical convection cell is present, with the center of the cell near the right vertical wall, exactly in the middle of the liquid layer. From the arrows on the velocity vectors, one can tell that the flow is moving in a counter-clockwise direction. It is also evident that there is no flow in the porous layer of the cavity. 

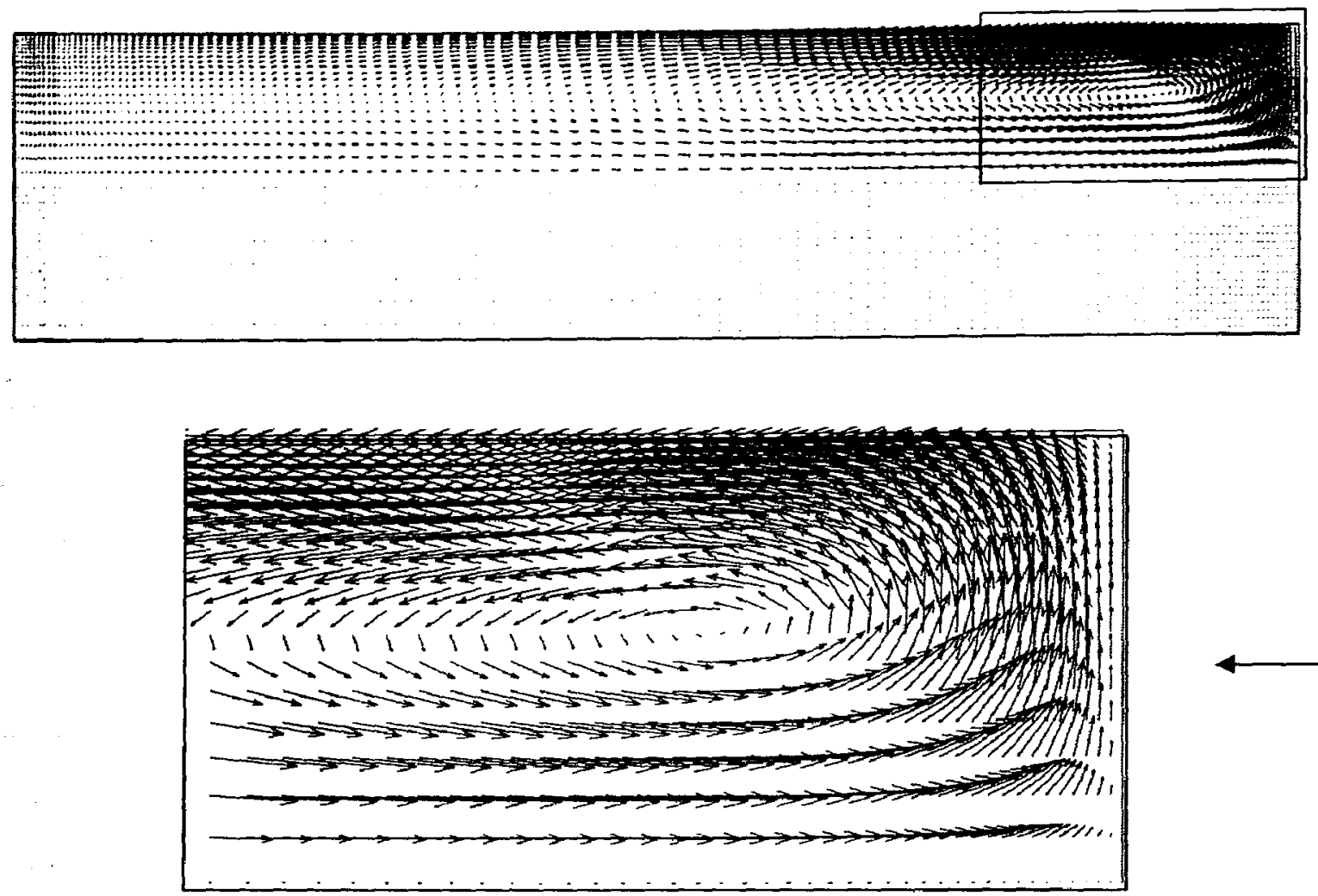

Figure 19 Buoyancy convection velocity plot with a cut on the $Z$-axis at 0.25 , displayed in the $X-Y$ plane $\left(R a_{L}=1000, M a=1, d_{1}=d_{2}=0.5, A R=4, B i=1, P r=7\right)$

To further investigate the similarity between the two and three-dimensional cases, the temperature and velocity profiles are plotted at the free surface of the cavity. Figure 20a, shows the temperature versus the X-coordinate, or the aspect ratio, for the two cases studied. It is clearly seen from the plot that the temperature distribution follows the same pattern for both graphs. Further, in Figure 20b, the U component of velocity is plotted at the free surface. The trend of the two plots is the same with the maximum twodimensional velocity equal to $4.56 \times 10^{-2}$ and the maximum three-dimensional velocity equal to $3.92 \times 10^{-2}$. The small difference in these magnitudes can be attributed to the fact that the convection is slowed in the three-dimensional cavity by the effects of the additional vertical walls, as noted by Dauby and Lebon [12] in their three-dimensional cavity study. This effect is further illustrated by plotting the $U$ component of velocity, with cuts made closer to the vertical wall at $Z=0.1$ and $Z=0.01$, with the velocity decreasing accordingly. 


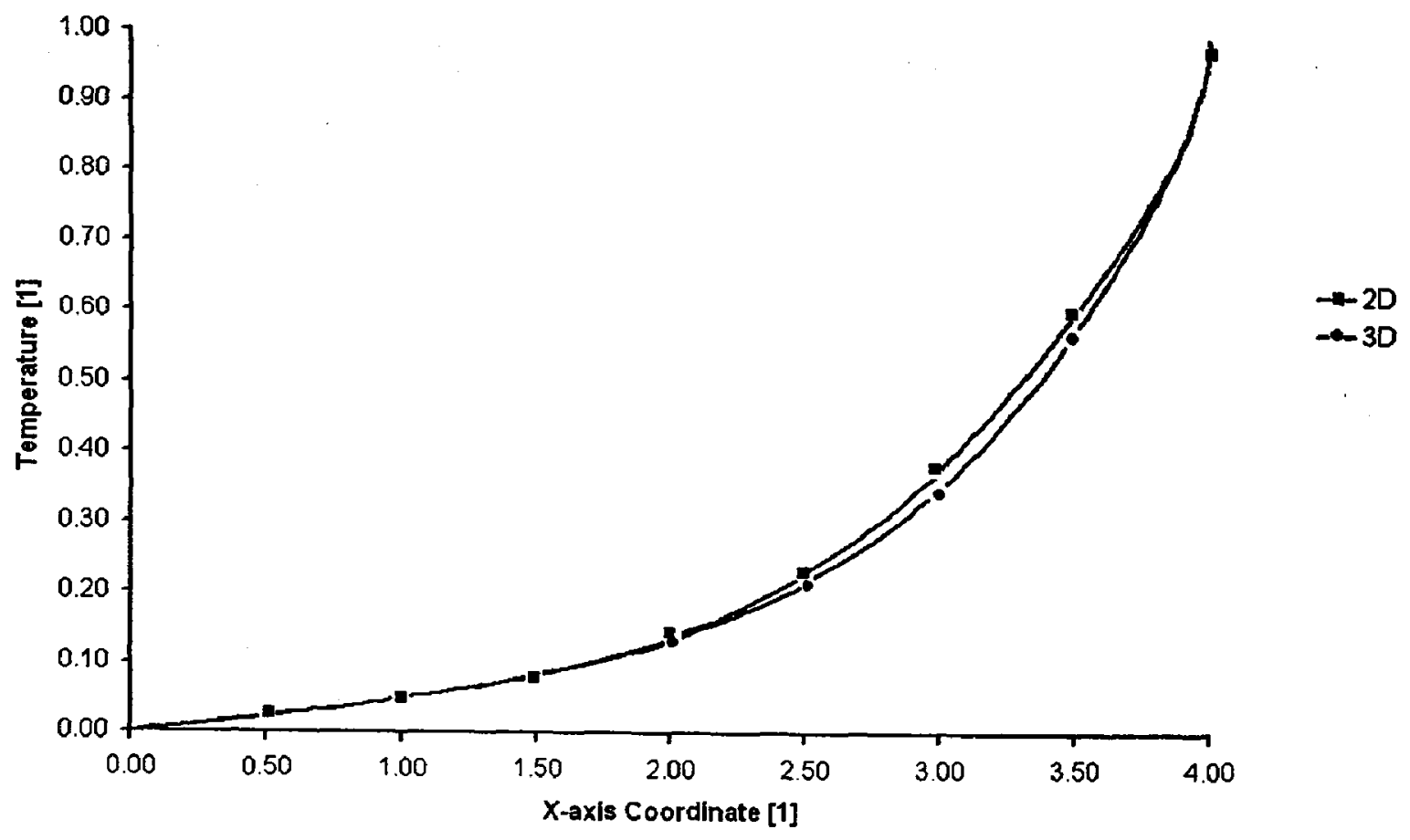

a)

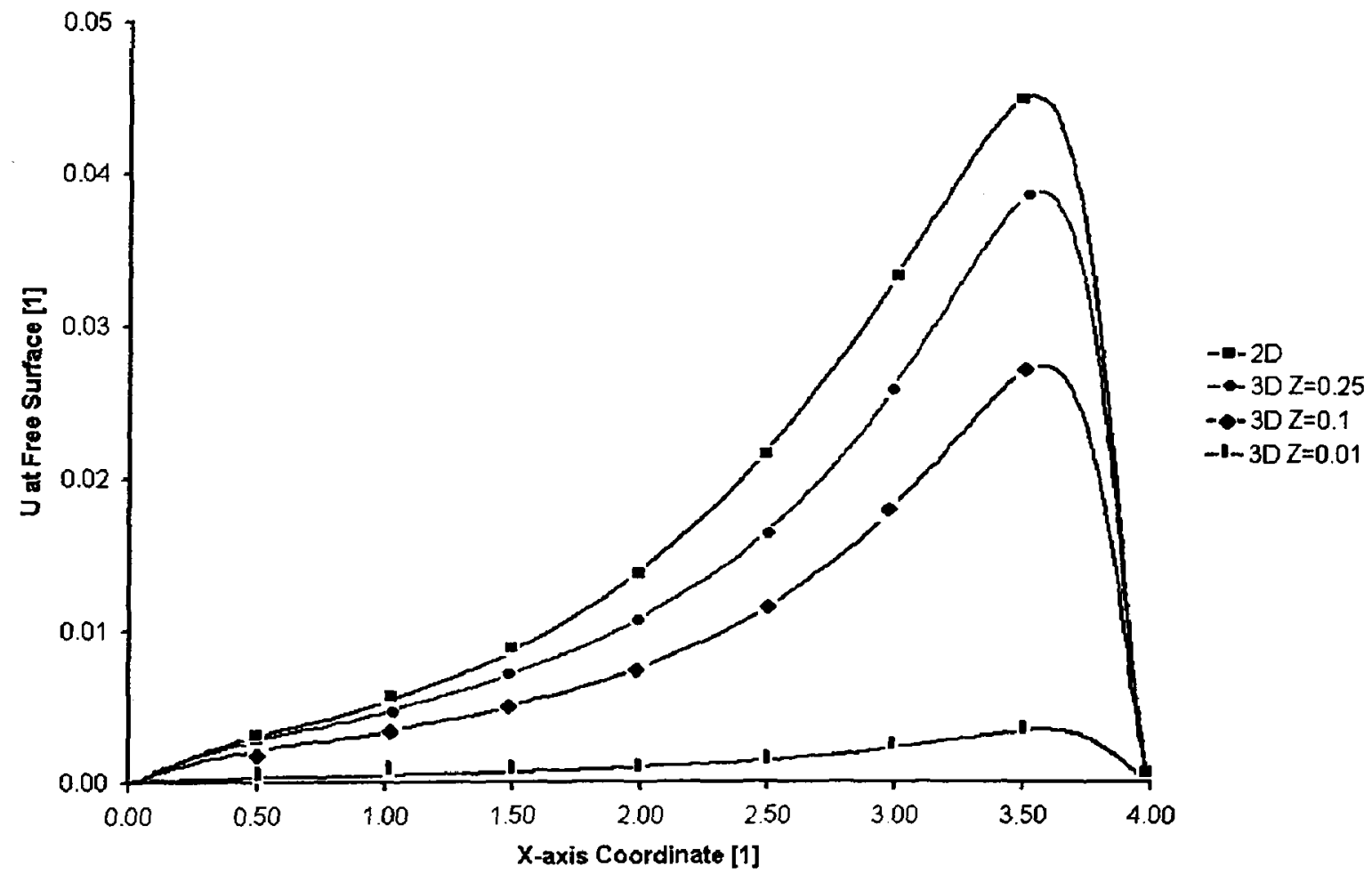

b)

Figure 20 Comparison of two and three-dimensional buoyancy convection

$$
\left(\mathrm{Ra}_{\mathrm{L}}=1000, \mathrm{Ma}=1, \mathrm{AR}=4, \mathrm{Bi}=1, \mathrm{Pr}=7\right) \text { : }
$$
a) Temperature at free surface
b) $U$ at free surface 
A comparison has also been performed for thermocapillary flow in a two and threedimensional laterally heated cavity. The two-dimensional case chosen for the analysis included a Marangoni number of 60000 , equal liquid and porous layer thicknesses $d_{l}=\mathrm{d}_{2}$ $=0.5$ to ensure flow in the liquid layer, and an aspect ratio of 4 . The two-dimensional results can be seen in Figure 13b. The Biot number is kept constant at $B i=1$ for all cases studied. From the velocity vector plot in Figure 21, it is easy to see that a similar flow pattern developed, with the center of the larger cell closer to the hot vertical wall and free surface. The presence of a second cell further to the left cannot easily be seen from the vector plot; however it is seen in Figure $22 \mathrm{~b}$ as a velocity increase at the free surface in that area. Therefore, for both the two and three-dimensional thermocapillary flow, multi cellular flow develops as the aspect ratio is increased.
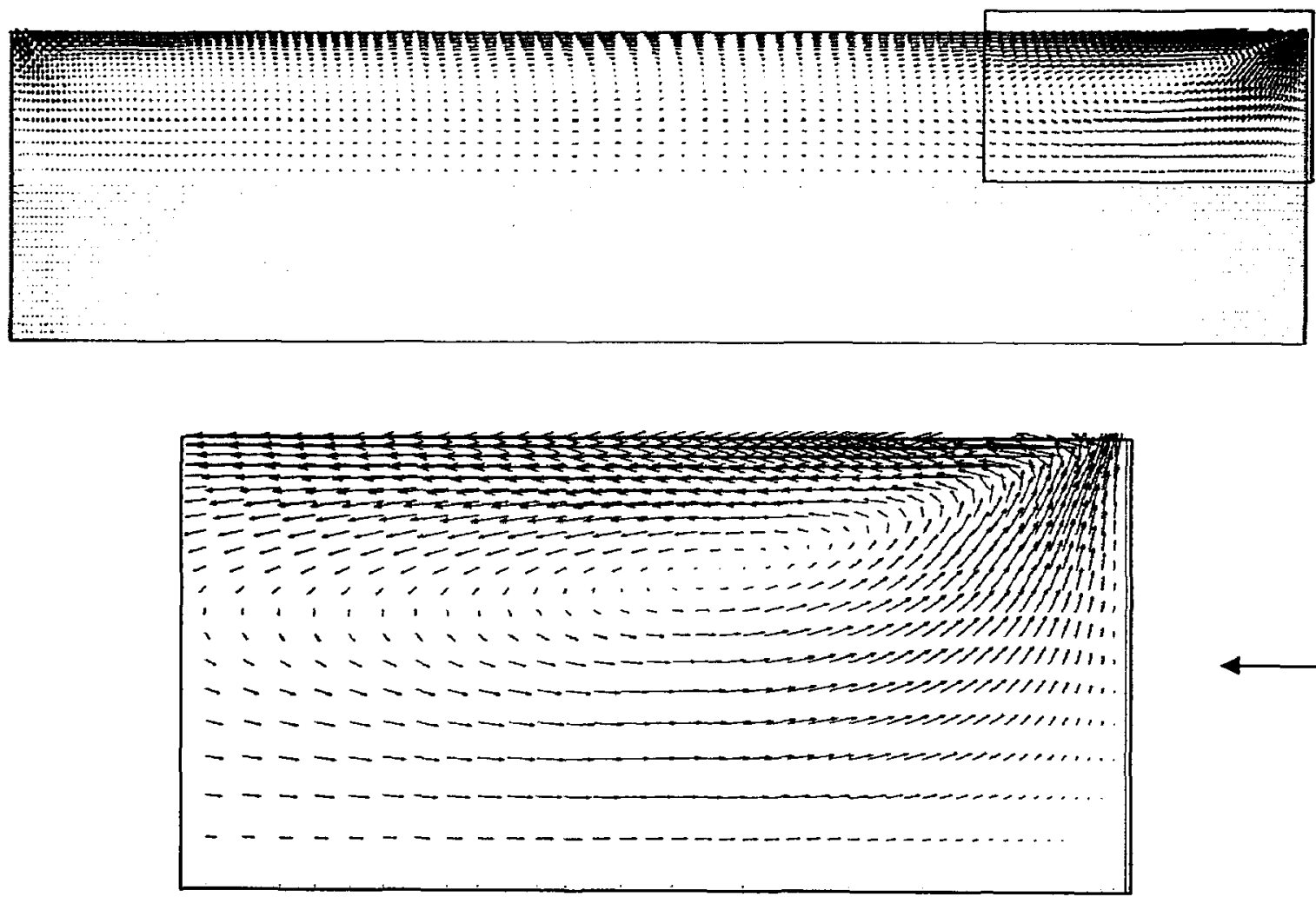

Figure 21 Thermocapillary convection velocity plot with a cut on the $\mathrm{Z}$-axis at 0.25 , displayed in the $X-Y$ plane $\left(\mathrm{Ma}=60000, \mathrm{Ra}_{\mathrm{L}}=1, \mathrm{~d}_{1}=\mathrm{d}_{2}=0.5, \mathrm{AR}=4, \mathrm{Bi}=1, \mathrm{Pr}=7\right)$ 
Figure 22 displays the comparison of the temperature at the free surface and the $U$ velocity component at the free surface for thermocapillary convection for both the two and three-dimensional geometry. The small oscillations at the start of the plot for the three-dimensional case are due to the large temperature gradient in that region and boundary layer problems at the vertical wall. The temperature plot in Figure 22a shows a similar trend for both cases, with the three-dimensional temperature being slightly higher in the first half of the cavity closer to the hot wall. From Figure 22b, it is seen that in the three-dimensional case a second cell also develops and is shifted closer to the hot wall, as compared to the two-dimensional case. This shift of the second cell towards the hot wall could be attributed to the fact that, for the three-dimensional cavity, a third cell is beginning to form near the cold vertical wall. Therefore, it is found that, in the threedimensional cavity, multi cellular flow formation occurs earlier, for smaller aspect ratios, than in the two-dimensional case. The maximum non-dimensional velocity in the $\mathrm{X}$ direction is equal to 274 and 281 for two and three-dimensional convection, respectively. Thermocapillary convection is visibly a stronger driving force for fluid flow when compared to buoyancy convection alone in a laterally heated cavity. 


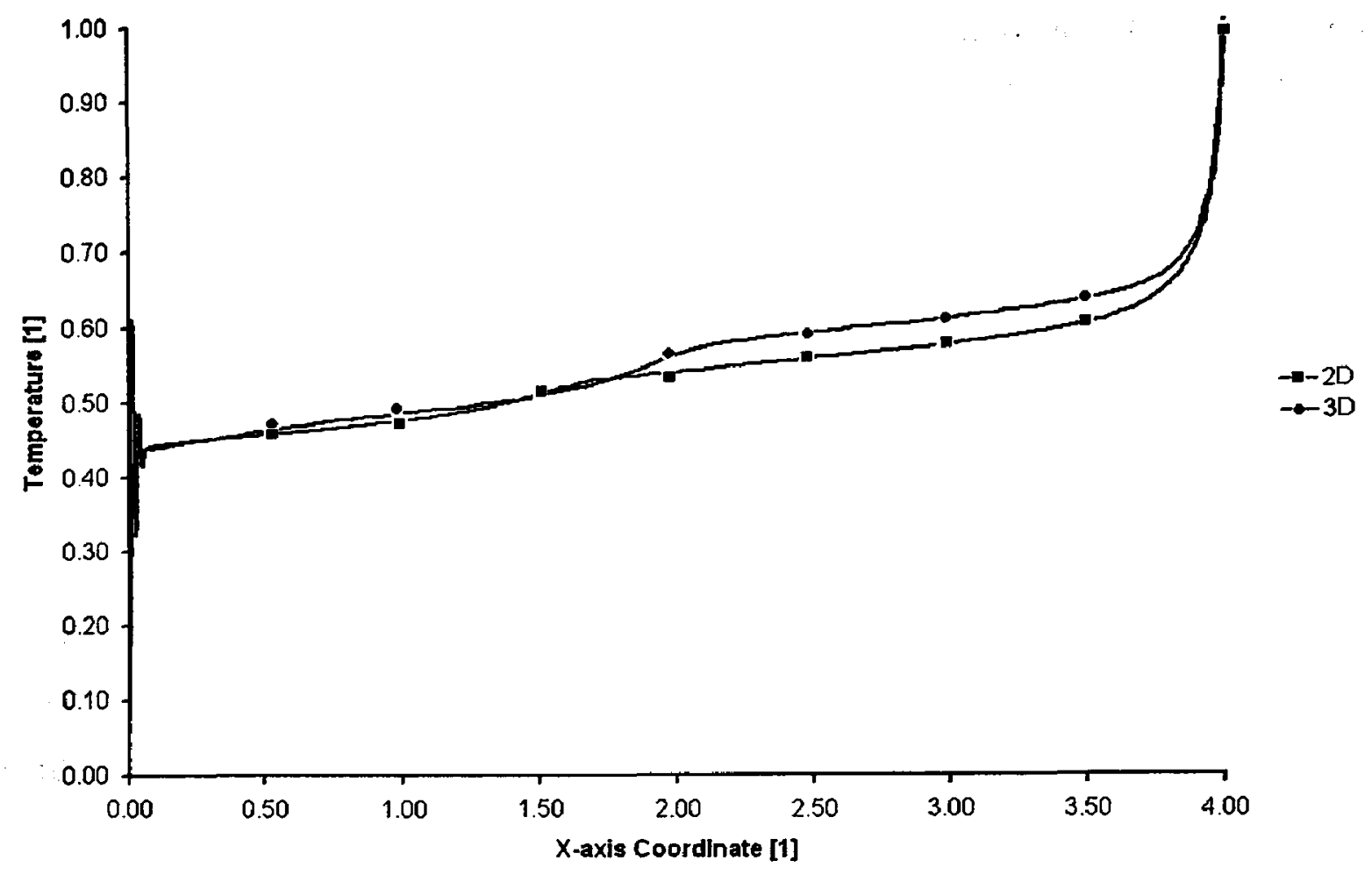

a)

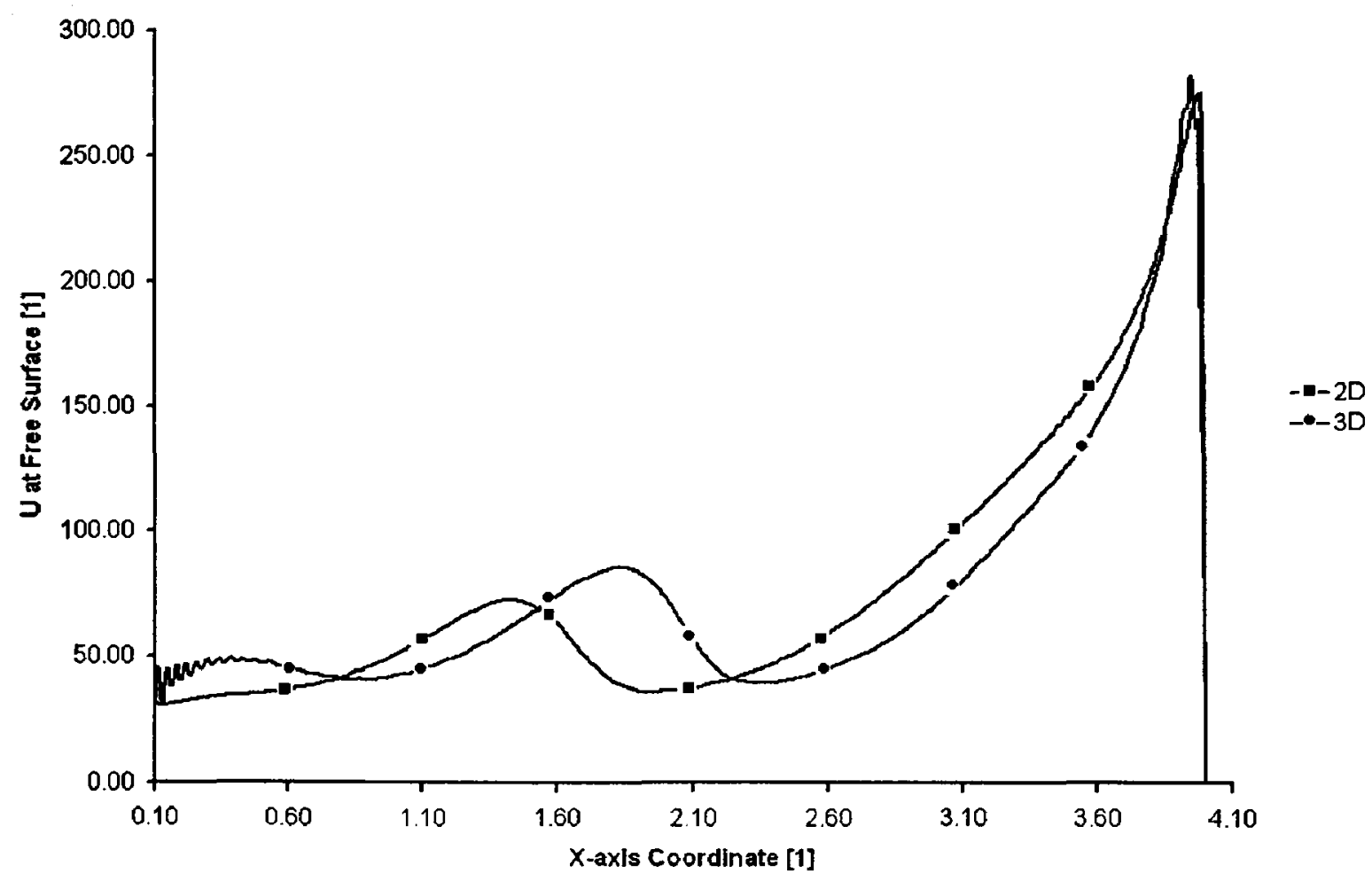

b)

Figure 22 Comparison of two and three-dimensional thermocapillary convection

$$
\left(\mathrm{Ma}=60000, \mathrm{~d}_{1}=\mathrm{d}_{2}=0.5, \mathrm{AR}=4, \mathrm{Bi}=1, \mathrm{Pr}=7\right) \text { : }
$$

a) Temperature at the free surface b) $U$ at the free surface 
The last case studied for lateral heating, is that of combined thermocapillary and buoyancy convection. The two-dimensional case that has been chosen for the analysis has the Marangoni number of $M a=60000$ and the liquid Rayleigh number of $R a_{L}=$ 1000 , as indicated in Figure 17b. The cavity is studied for equal thicknesses $d_{1}=d_{2}=0.5$ and an aspect ratio of $A R=4$. Multi cellular flow is present in the three-dimensional cavity, with the center of the cell close to the hot wall and free surface.
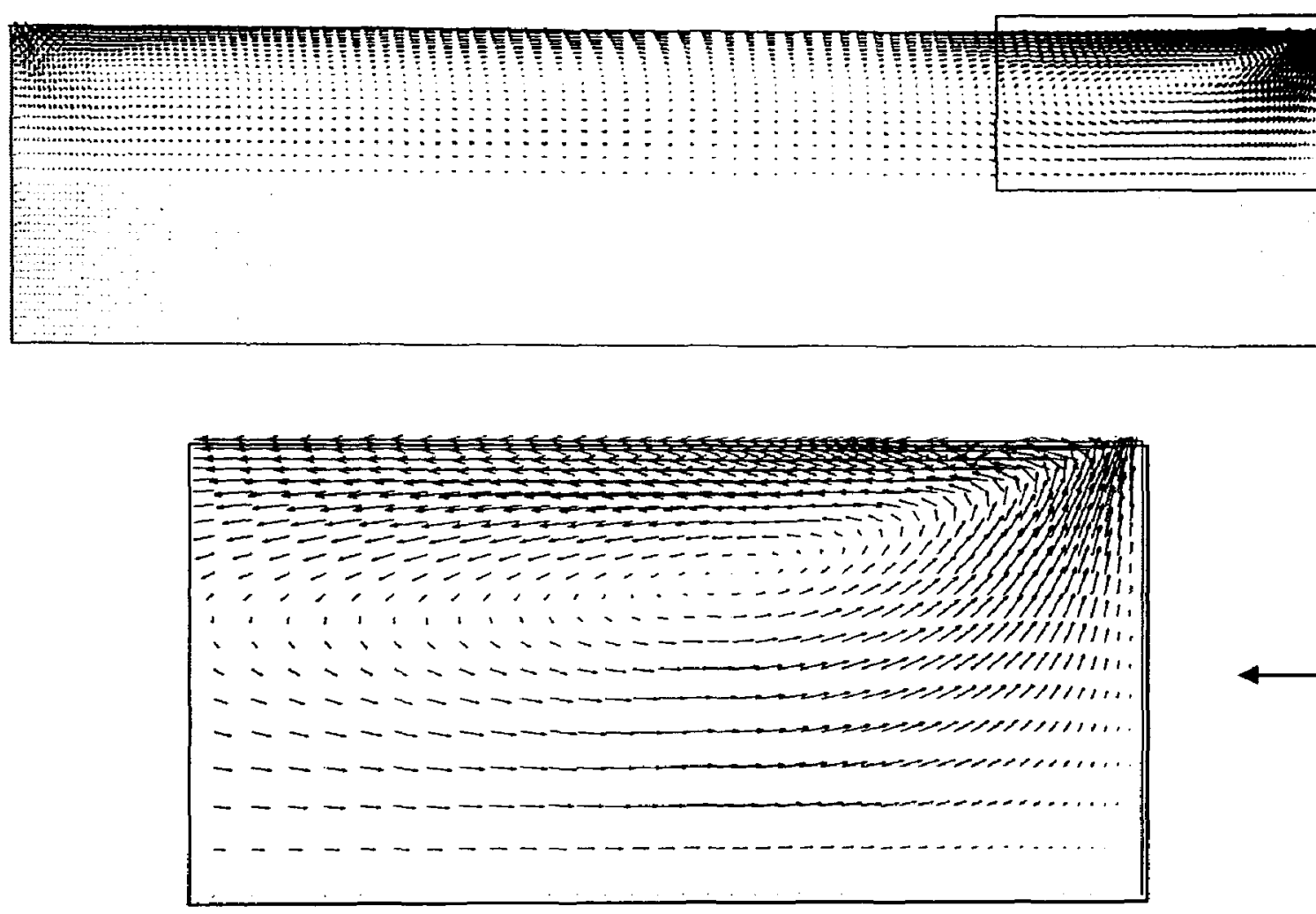

Figure 23 Combined thermocapillary and buoyancy convection velocity plot with a cut on the $Z$-axis at 0.25 , displayed in the $X-Y$ plane

$$
\left(\mathrm{Ma}=60000, \mathrm{Ra}_{\mathrm{L}}=1000, \mathrm{~d}_{1}=\mathrm{d}_{2}=0.5, \mathrm{AR}=4, \mathrm{Bi}=1, \operatorname{Pr}=7\right)
$$

Figure 24 presents the plots of the temperature and the $U$ velocity component at the free surface for combined thermocapillary and buoyancy convection. The maximum nondimensional velocity for the two and three-dimensional convection is equal to 8.67 and 8.89 , respectively. These values are in close agreement with each other. However, once again in the three-dimensional flow, the second cell shifted closer to the hot wall. 


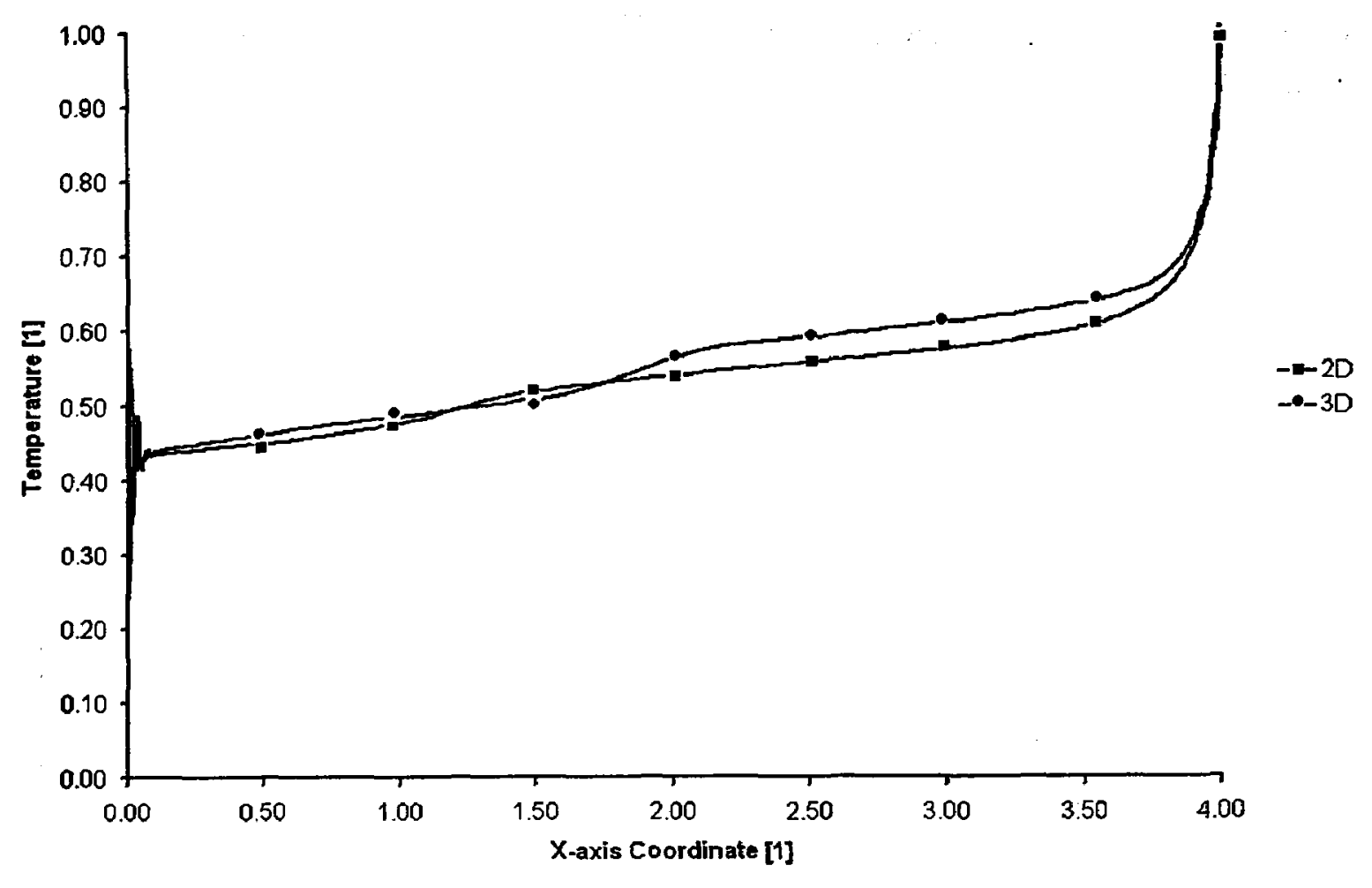

a)

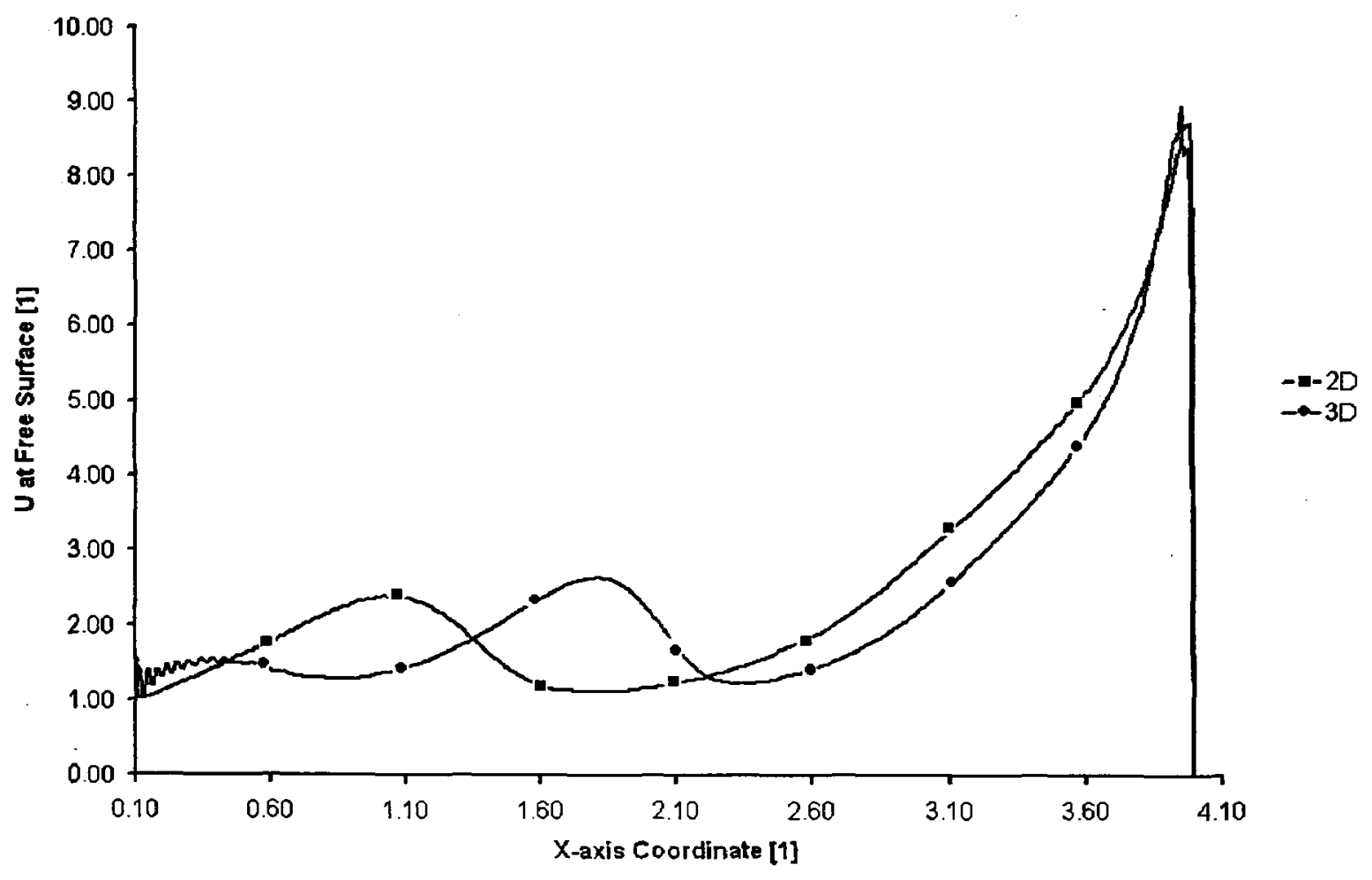

b)

Figure 24 Comparison of two and three dimensional combined buoyancy and thermocapillary convection ( $\mathrm{Ma}=60000, \mathrm{Ra}_{\mathrm{L}}=1000, \mathrm{~d}_{1}=\mathrm{d}_{2}=0.5, \mathrm{AR}=4, \mathrm{Bi}=1, \mathrm{Pr}=7$ ):

a) Temperature at the free surface b) $U$ at the free surface 


\subsubsection{Bottom Heating}

Thermocapillary flow in a bottom heated cavity has also been analyzed for similarity between two and three-dimensional convection in Figure 25. The case studied included thermocapillary convection with a Marangoni number of $M a=60000$, an aspect ratio of $A R=2$, and equal liquid and porous layer thicknesses $d_{1}=d_{2}=0.5$. As in the twodimensional model, the flow remained in the liquid layer, without penetrating into the porous one. Two counter-rotating cells formed with the highest velocity near the free surface.

Figure 26 shows a graph of the non-dimensional X-component of velocity, $U$, at the free surface for thermocapillary convection in a bottom heated cavity. The counter-rotating nature of the two cells can clearly be seen by the opposite velocities of the two cells. The trend in the velocities is also very similar for the two cases studied. The threedimensional model is in close agreement with the two-dimensional one. Therefore, for the evaporation analysis in the next chapter, only a two-dimensional cavity is studied for the lateral heating condition with thermocapillary convection. 

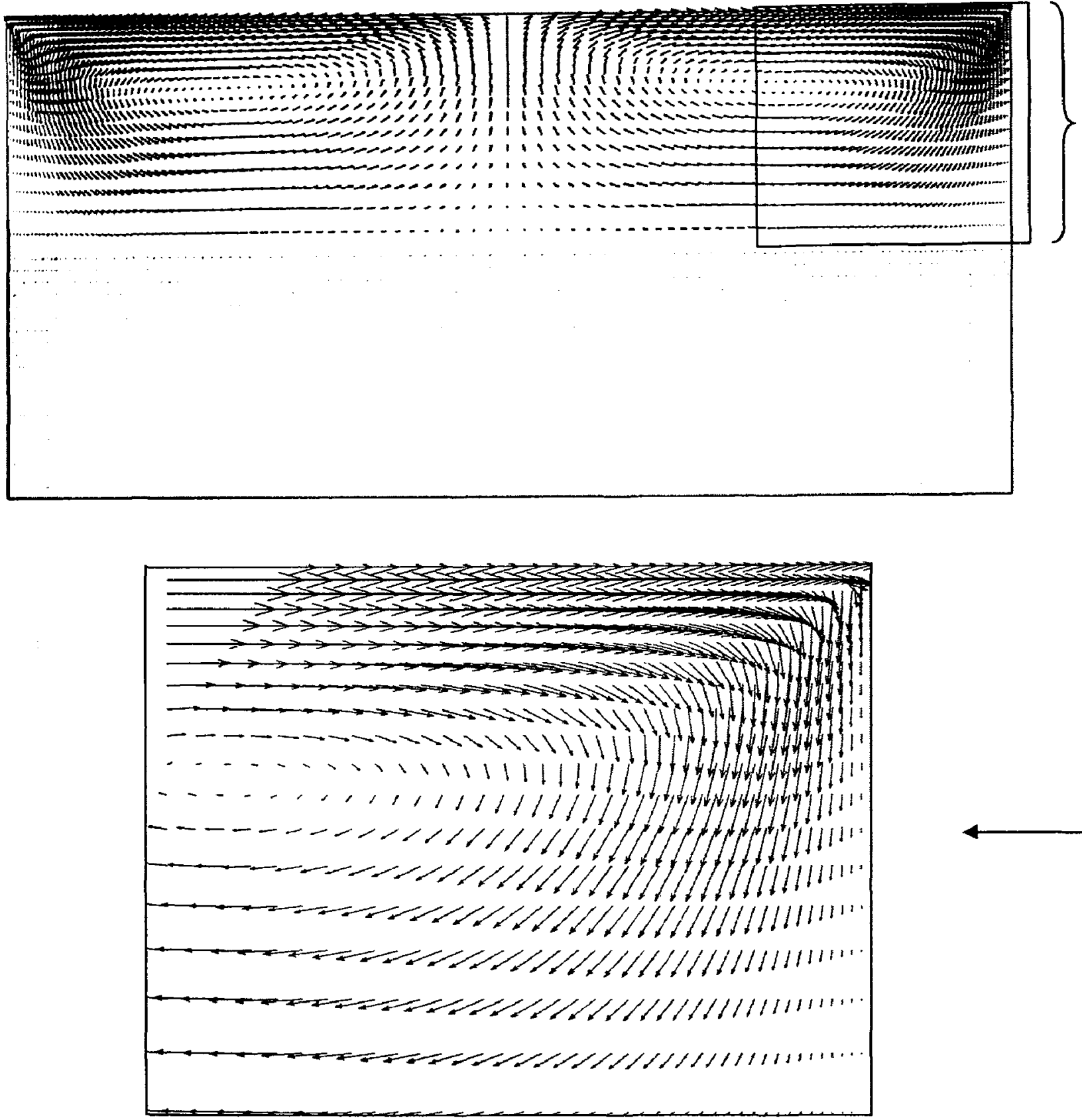

Figure 25 Thermocapillary convection velocity plot with a cut on the $Z$-axis at 0.25 , displayed in the $X-Y$ plane $\left(\mathrm{Ma}=60000, \mathrm{Ra}_{\mathrm{L}}=0.1, \mathrm{Ra} \mathrm{P}_{\mathrm{P}}=0.0055, \mathrm{~d}_{1}=\mathrm{d}_{2}=0.5, \mathrm{AR}=2, \mathrm{Bi}=1, \operatorname{Pr}=7\right)$ 


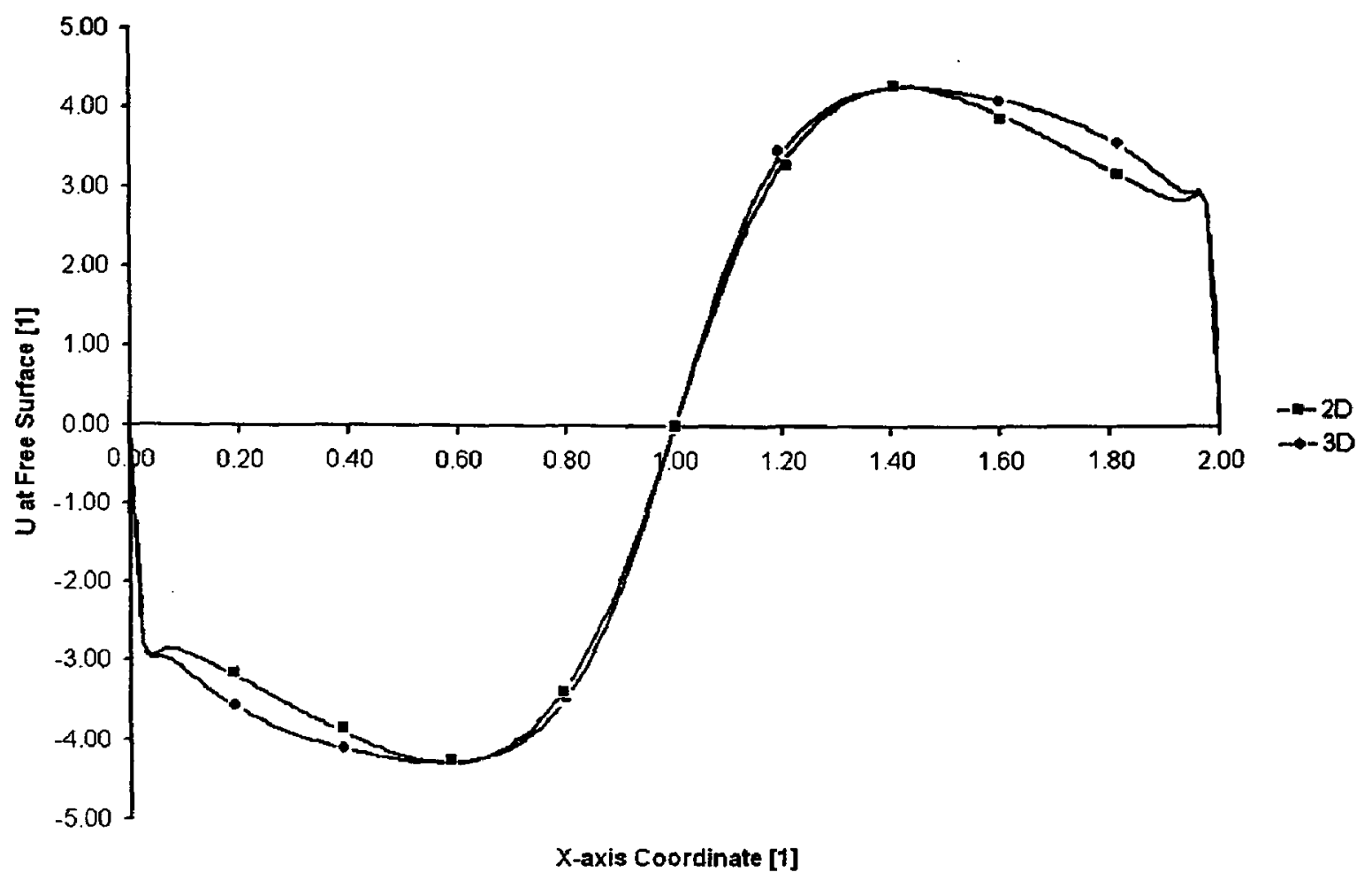

Figure 26 Comparison of two and three-dimensional thermocapillary convection $\left(\mathrm{Ma}=60000, \mathrm{Ra}_{\mathrm{L}}=0.1, \mathrm{Ra}_{\mathrm{P}}=0.0055, \mathrm{~d}_{1}=\mathrm{d}_{2}=0.5, \mathrm{AR}=2, \mathrm{Bi}=1, \mathrm{Pr}=7\right.$ ) 


\section{CHAPTER 4}

\section{Superposed Liquid and Porous Layer with Evaporation at Free Surface}

\subsection{Introduction}

The evaporation of the liquid occurs at the free surface, thus simultaneously removing heat and mass from the system at that interface. Numerically, only the liquid phase is being modeled with the vapour phase imposing some boundary conditions at the liquid vapour interface as described in section 2.7.3. The laterally heated thermocapillary convection case is studied for different aspect ratios, thickness ratios and Marangoni numbers. Evaporation is imposed on the free surface with a linear saturation temperature applied at the liquid-vapour interface.

\subsection{Lateral Heating}

Figure 27 displays the results for thermocapillary convection with evaporation in the liquid layer. The case is considered with the Marangoni number equal to 60000 . The liquid Rayleigh number is suppressed to ensure thermocapillary convection. To analyze the flow, the results are compared to those of the case without evaporation at the free surface, as shown in Figure 13. The phenomenon of evaporation produces some interesting results. Most evident is the fact that the location of the cell switches from the hot wall to the cold wall in fluid dominated flow, where the thickness of the liquid layer is equal to that of the porous. However, the fluid motion is still in the counter-clockwise direction. Also, as the aspect ratio is increased, multi cellular flow does not develop as in the case without evaporation. It is also noted that the flow becomes much stronger when the values of streamlines are compared. In the case without evaporation, for $A R=2$, the maximum streamline has a value of $1.05 \times 10^{1}$ as compared to that with evaporation of $2.81 \times 10^{1}$. For an aspect ratio of $A R=4$, the case without evaporation has a maximum streamline of $1.04 \times 10^{1}$, while with evaporation this increases to $2.03 \times 10^{1}$. It is evident that evaporation enhances the flow greatly when the fluid flow is constrained to the liquid 
layer. Similar findings have been shown by Ward et al. [21], where the evaporation enhanced Marangoni convection.

When comparing the case where the flow enters the entire cavity with a porous layer thickness of $d_{2}=0.97$, another interesting fact is observed. For the case of convection without evaporation, the maximum streamline value for $A R=2$, is $1.0 \times 10^{-2}$. This decreases to $6.4 \times 10^{-3}$ when evaporation is added at the liquid-vapour interface. Similarly for $A R=4$, without evaporation, the maximum streamline value is $9.82 \times 10^{-3}$, and with evaporation this decreases to $3.98 \times 10^{-3}$. The strength of the flow in the porous layer also decreases as the aspect ratio is increased. A conclusion can be made that evaporation does not have a significant affect on flow that is occurring predominantly in the porous layer.

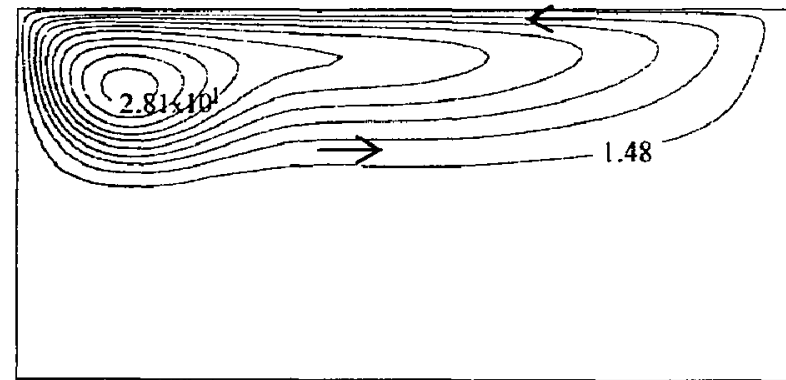

$\Delta \Psi=2.96$

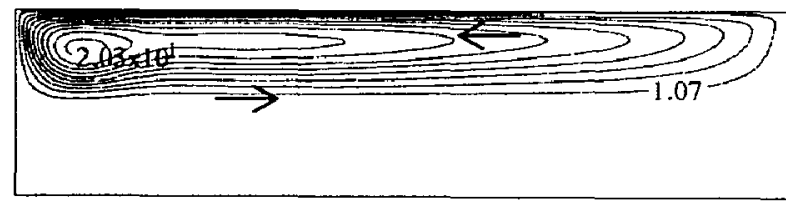

$\Delta \Psi=2.14$

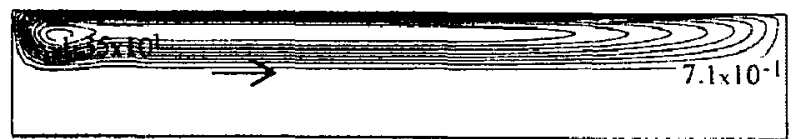

$\Delta \Psi=1.42$

$\mathrm{d}_{1}=\mathrm{d}_{2}=0.5$

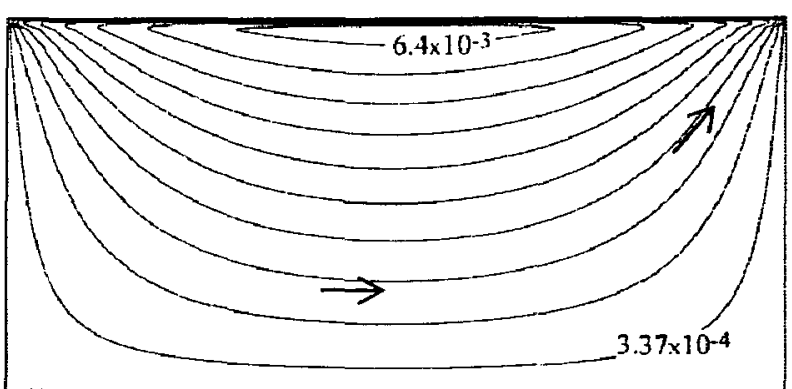

$\Delta \Psi=6.75 \times 10^{-4}$

a)

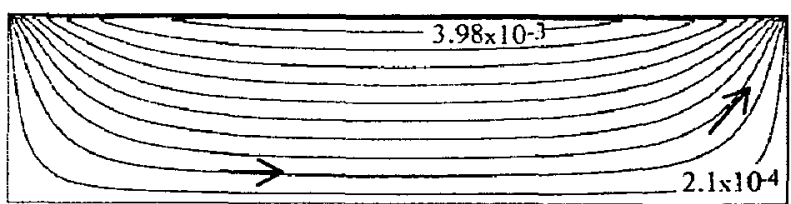

$\Delta \Psi=4.19 \times 10^{-4}$

b)

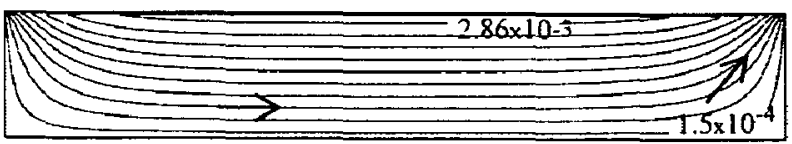

$\Delta \Psi=4.19 \times 10^{-4}$

c)

$\mathrm{d}_{1}=0.03, \mathrm{~d}_{2}=0.97$

Figure 27 Thermocapillary convection with evaporation in the liquid layer
$\left(\mathrm{Ma}=60000, \mathrm{Ra}_{\mathrm{L}}=1, \mathrm{Pr}=7\right)$ : a) $\mathrm{AR}=2$
b) $\mathrm{AR}=4$
c) $\mathrm{AR}=6$ 
Also analyzed is the non-dimensional X-component of velocity, $U$, at the free surface for the case without evaporation and with evaporation. This comparison is shown in Figure 28 for $A R=2$ in part a and for $A R=4$ in part b. For the case when the aspect ratio is equal to 2 , the velocity is seen to increase significantly when evaporation is included in the liquid layer. The case without evaporation has a maximum velocity value of 284 , and with evaporation this increases to 375 . The evaporation is definitely enhancing the flow when the convection is limited to the liquid layer. For the case of $A R=4$, the velocity actually decreases when evaporation is present. The value changes from 274 without evaporation to 251 with evaporation. This can be explained by the fact that in the case without evaporation, multi cellular flow is present, but with evaporation, multiple cells do not develop.

Figure 29 shows a comparison of the X-component of velocity, U, plotted on the vertical median of the cavity for convection with and without evaporation. A parabolic trend in the plot is observed which was also noted by Villers and Platten [10] for flow in a liquid layer. It is also confirmed that there is no flow in the porous layer with a zero velocity throughout the vertical cut below a $\mathrm{Y}$-axis coordinate value of 0.5 . The direction of the flow is also similar in both cases with the velocity in the counter-clockwise direction. From the plot, it is clear that evaporation enhances the velocity of the convection. For the case without evaporation, the surface velocity in the negative $\mathrm{X}$-direction is 92 , and with evaporation this increases to 298 . 


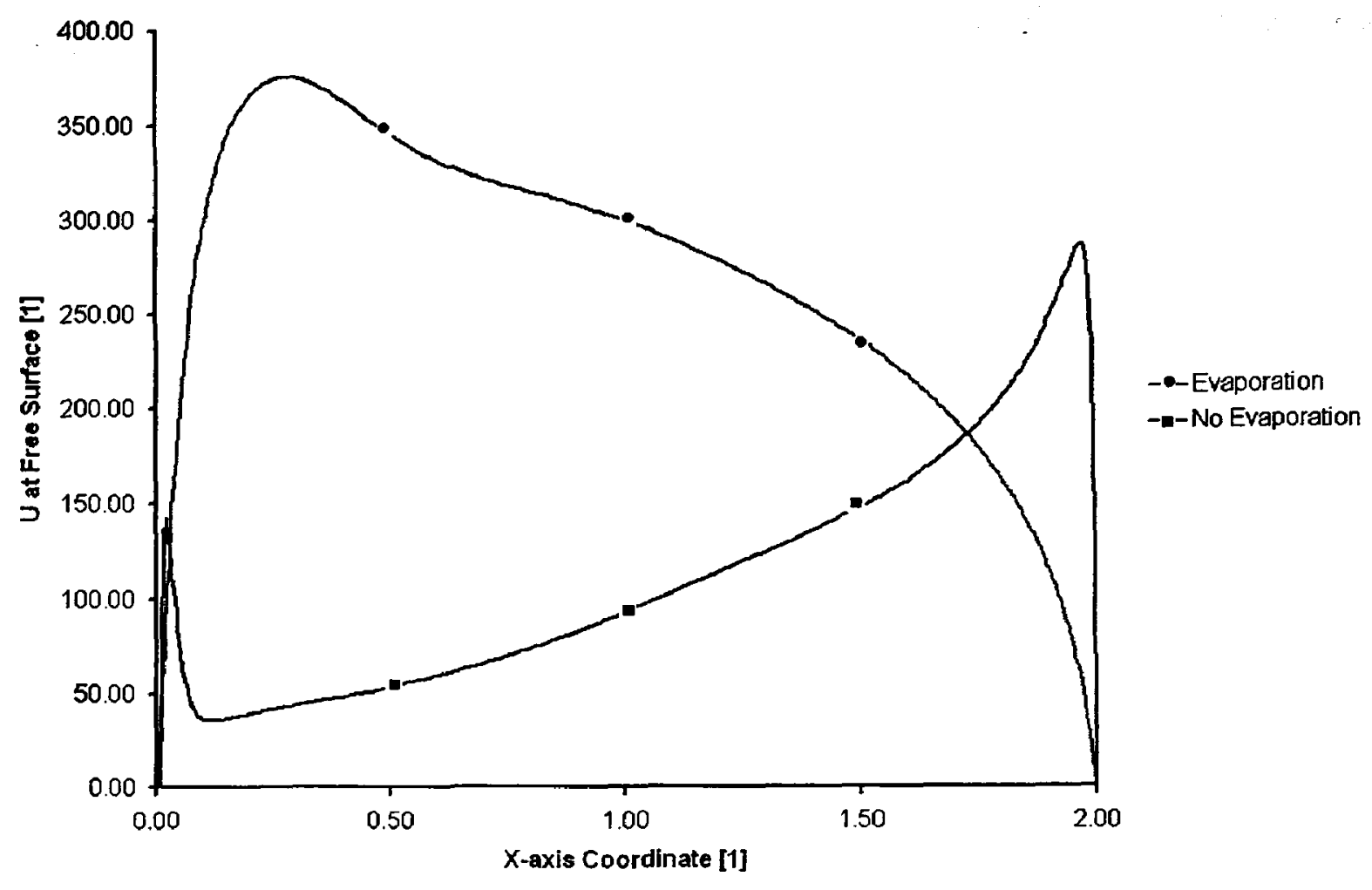

a)

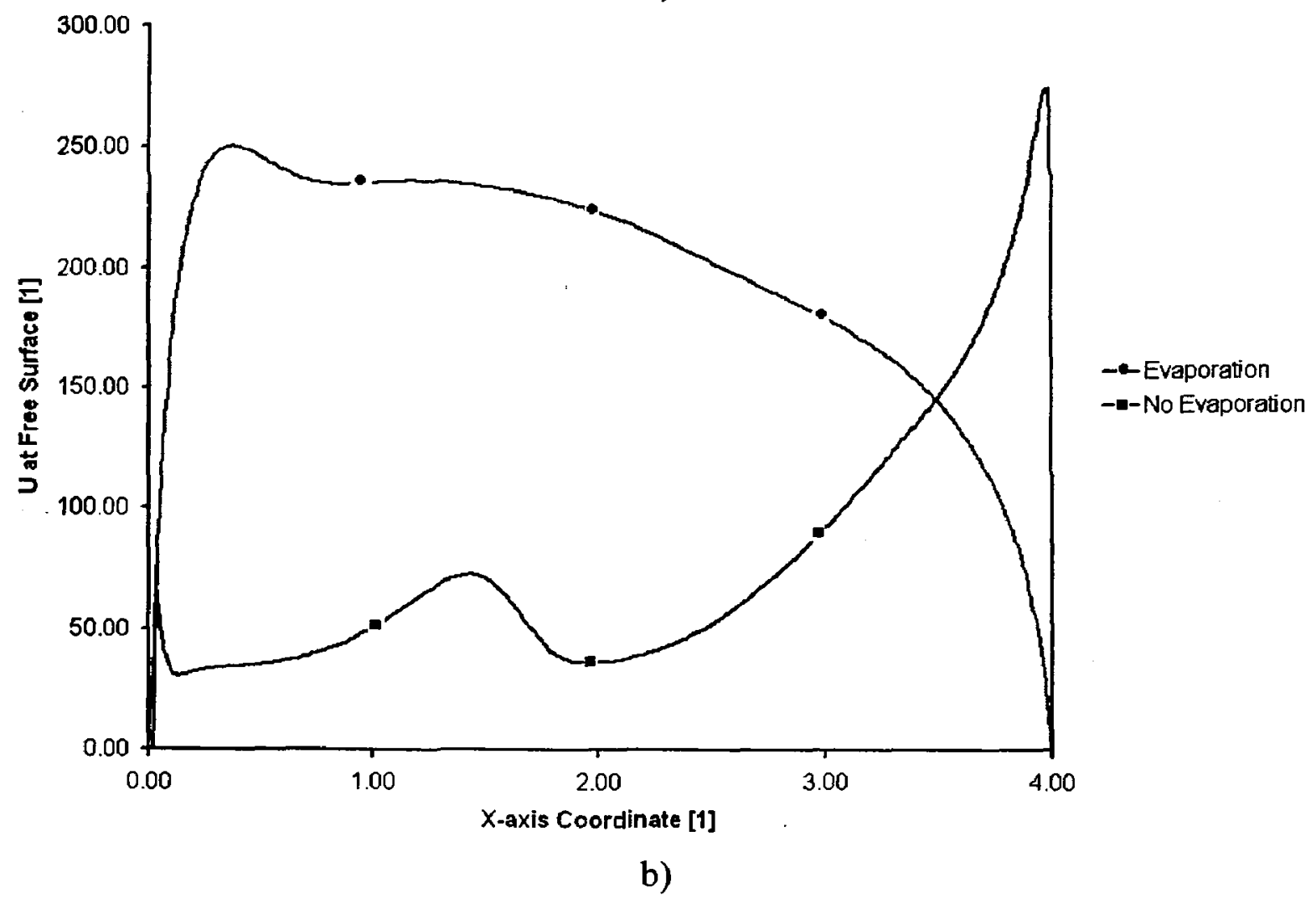

Figure 28 Comparison of thermocapillary convection with and without evaporation in the liquid layer $\left(\mathrm{Ma}=60000, \mathrm{Ra}_{\mathrm{L}}=1, \mathrm{~d}_{1}=\mathrm{d}_{2}=0.5, \mathrm{Pr}=7\right)$ : a) $\mathrm{AR}=2 \quad$ b) $\mathrm{AR}=4$ 


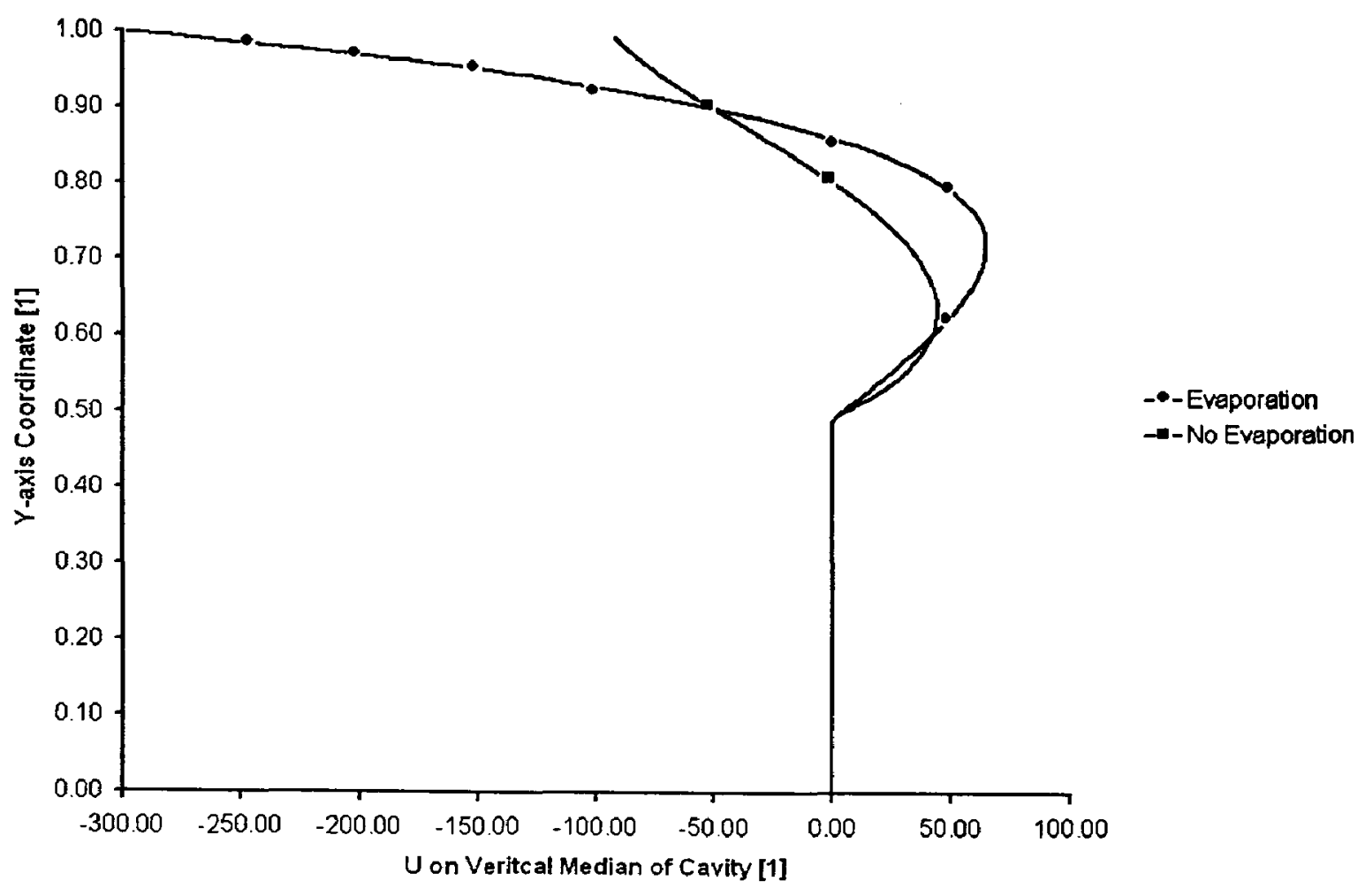

Figure 29 Comparison of $U$ component of velocity on vertical median of cavity for thermocapillary convection with and without evaporation

$$
\left(\mathrm{Ma}=60000, \mathrm{Ra}_{\mathrm{L}}=1, \mathrm{~d}_{1}=\mathrm{d}_{2}=0.5, \mathrm{Pr}=7\right)
$$

Thermocapillary flow with evaporation at the free surface is also investigated for different Marangoni numbers as shown in Figure 30. The Marangoni number increases from 60000 , to 80000 and then to 100000 . The flow is limited to the liquid layer by choosing equal liquid and porous layer thicknesses $d_{I}=d_{2}=0.5$. The effect of increasing the Marangoni number is seen as enhanced flow in the liquid layer of the cavity. This can also be visualized by studying Figure 31, where the non-dimensional X-component of velocity has been plotted for the various Marangoni numbers. The maximum velocity for $M a=60000$ is 375 . This increases to 469 for $M a=80000$, and 553 for $M a=100000$. Therefore, increasing the Marangoni number enhances thermocapillary convection in a liquid layer with evaporation at the liquid-vapour interface. 


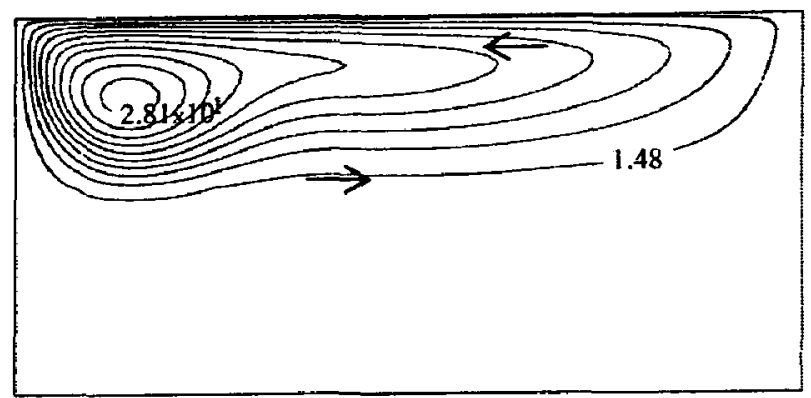

a) $\Delta \Psi=2.96$

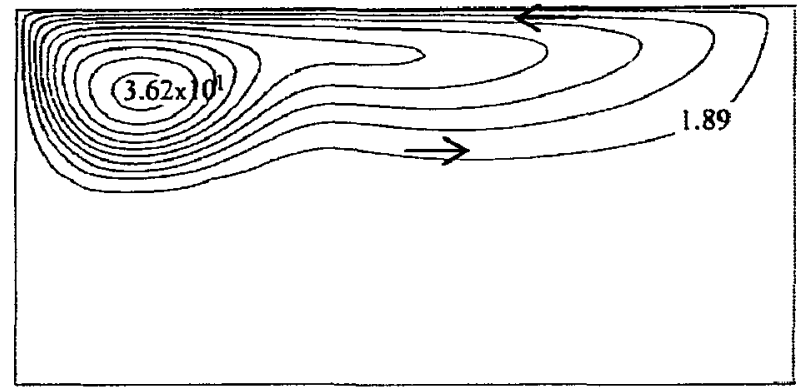

b) $\Delta \Psi=3.8$

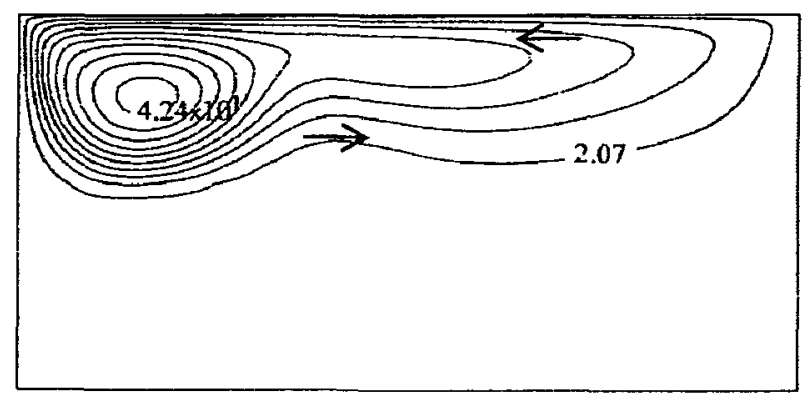

c) $\Delta \Psi=4.48$

Figure 30 Thermocapillary convection with evaporation in the liquid layer for different Marangoni numbers $\left(\mathrm{Ra}_{\mathrm{L}}=1, \mathrm{~d}_{1}=\mathrm{d}_{2}=0.5, \mathrm{AR}=2, \mathrm{Pr}=7\right)$ :
a) $\mathrm{Ma}=60000$
b) $\mathrm{Ma}=80000$
c) $\mathrm{Ma}=100000$ 


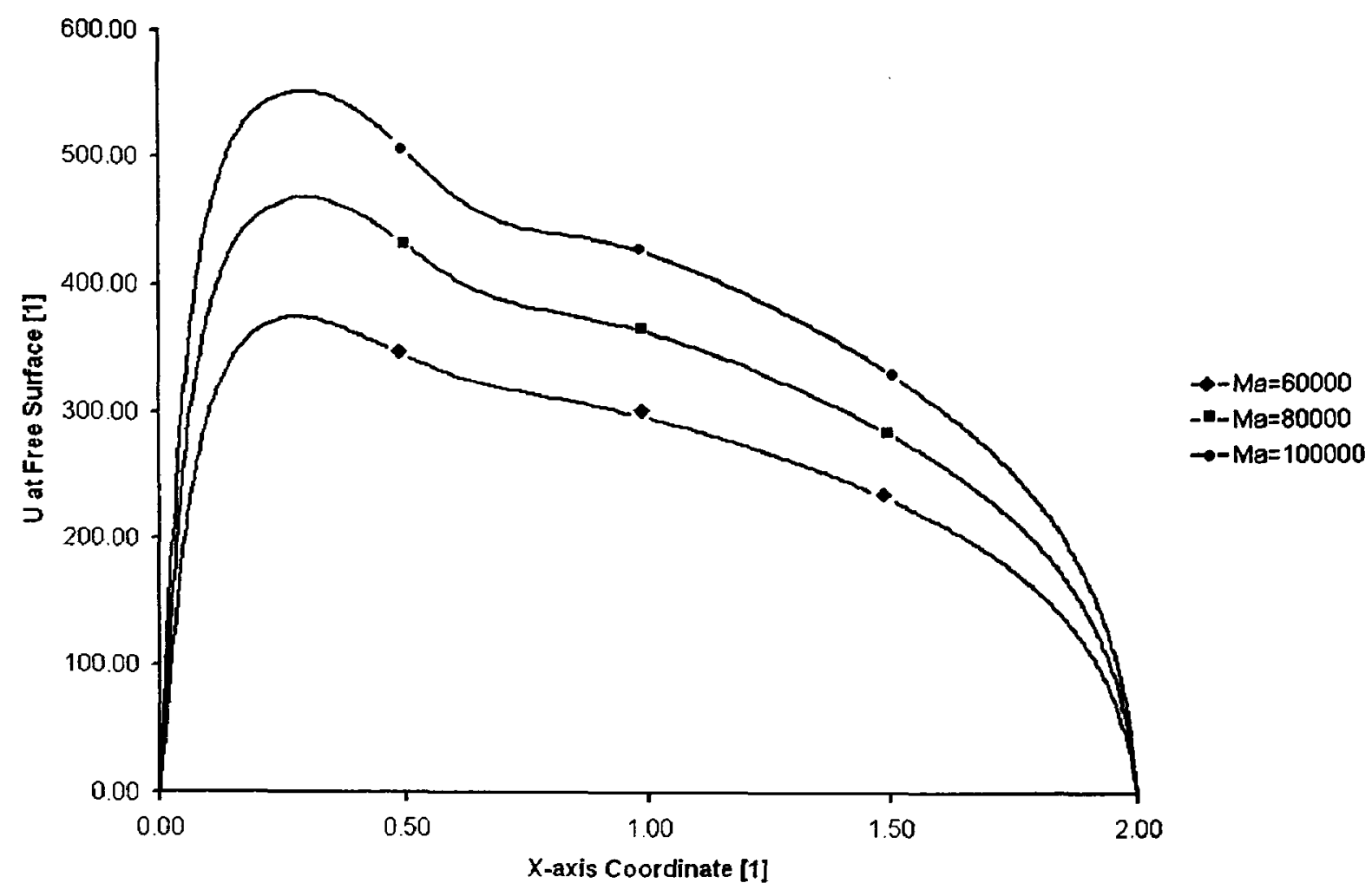

Figure 31 Thermocapillary convection with evaporation in the liquid layer for different Marangoni numbers $\left(\mathrm{Ra}_{\mathrm{L}}=0, \mathrm{~d}_{1}=\mathrm{d}_{2}=0.5, \mathrm{AR}=2, \mathrm{Pr}=7\right)$

In Figure 32, a comparison of the X-direction component of velocity, $U$, is again plotted on the vertical median of the cavity. In this case, the trend is examined for different values of Marangoni number in the case with evaporation at the free surface. The graph once again displays the parabolic trend that was observed by Villers and Platten [10] for a liquid layer. The $\mathrm{X}$-component of velocity is compared at the free surface when $\mathrm{Y}=1$, for Marangoni numbers of 60000,80000 and 100000 . The velocity values in the negative $\mathrm{X}$-direction are 298, 365 and 427, respectively. An increased Marangoni number is found to enhance the flow. 


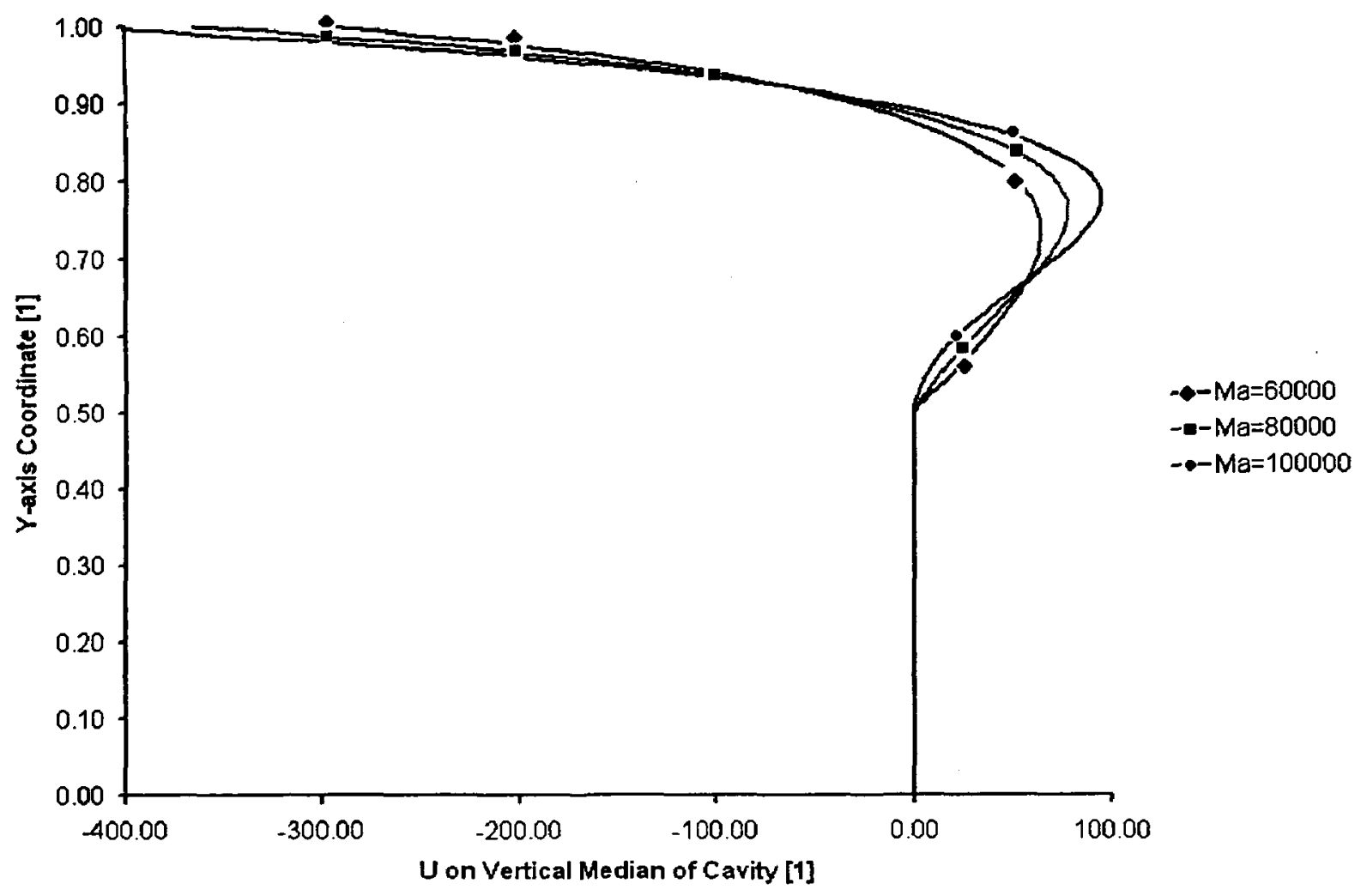

Figure 32 Comparison of $U$ component of velocity on vertical median of cavity for thermocapillary convection with evaporation $\left(\operatorname{Ra}_{\mathrm{L}}=1, \mathrm{~d}_{1}=\mathrm{d}_{2}=0.5, \mathrm{AR}=2, \mathrm{Pr}=7\right)$ 


\section{CHAPTER 5}

\section{Conclusion}

In this thesis, buoyancy, thermocapillary and combined buoyancy-thermocapillary convection has been studied in detail. A rectangular finite cavity has been considered with bottom and lateral heating conditions. Several cases have been examined and they include: two-dimensional geometry, three-dimensional geometry and the effect of evaporation applied to the liquid-vapour interface.

For buoyancy convection with bottom heating, it has been confirmed that the flow switches from fluid layer dominated to porous layer dominated convection upon reaching a critical porous layer thickness value of $d_{2}=0.9$. The flow occupied the entire porous cavity when this critical value was exceeded. For thermocapillary convection with bottom heating, it was confirmed that the critical porous layer thickness value is 0.96 . Below this value, the flow was limited to the liquid layer, and above it, the flow switched to the porous layer. For porous layer dominated thermocapillary flow, it was found that increasing the aspect ratio does not increase the number of cells in the cavity. But when the flow was limited to the liquid layer only, multi cellular convection did form as the aspect ratio was increased. For buoyancy convection, the opposite was found to be true, with multiple cells forming according to the aspect ratio in porous dominated flow.

The flow was found to behave in a similar fashion in the cavity with the lateral heating condition. The flow switched from fluid layer to porous layer dominated convection upon the critical value of porous layer thickness. The aspect ratio was also found to have an effect on the number of convective cells in the liquid layer for thermocapillary convection, with multiple cells forming accordingly. Thermocapillary flow was found to be stronger in nature when compared to the buoyancy flow in the lateral heating condition. For combined buoyancy and thermocapillary flow, the Marangoni effect was found to enhance the flow in the cavity. 
Upon comparing the results from the two and three-dimensional convection cases, it was verified that the two-dimensional flow model is a good representation of the threedimensional situation. However, for the laterally heated cavity with thermocapillary convection, a greater number of cells were found to occur in the three-dimensional model.

Lastly, evaporation was applied to the liquid-vapour interface of a laterally heated cavity with thermocapillary convection. An interesting phenomenon was discovered, in which the vortex of the convective cell switched from the hot side to the cold side when evaporation was applied. Evaporation was also found to enhance the Marangoni convection significantly, as was shown through the analysis of the streamlines and velocity profiles at the free surface.

For further study, it would be interesting to analyze the model for different heating conditions and include a different fluid with a different Prandtl number. It would also be interesting to observe the effect of tilted gravity and aspect ratio. It might also be useful to additionally study evaporation in a three-dimensional cavity. 


\section{APPENDIX A \\ Non-Dimensional Analysis of Governing Equations}

The following dimensionless variables are substituted into the dimensional equations in order to render them non-dimensional:

$$
\begin{gathered}
U=\frac{u}{u_{o}}, \quad V=\frac{v}{u_{o}}, \quad W=\frac{w}{u_{o}}, \quad X=\frac{x}{L}, \quad Y=\frac{y}{L}, \quad Z=\frac{z}{L}, \quad P=\frac{p \cdot L}{\mu \cdot u_{o}}, \\
\theta=\frac{T-T_{C}}{T_{H}-T_{C}}, \Delta T=T_{H}-T_{C}, \quad L=d_{1}+d_{2}, \quad \operatorname{Re}=\frac{\rho \cdot u_{o} \cdot L}{\mu}, \quad \operatorname{Pr}=\frac{v}{\alpha}, \quad D a=\frac{\kappa}{L^{2}}
\end{gathered}
$$

\section{B.1 Bottom Heating}

\section{Liquid Layer}

Continuity Equation

$$
\left[\frac{\partial u}{\partial x}+\frac{\partial v}{\partial y}+\frac{\partial w}{\partial z}\right]=0
$$

The dimensionless variables are introduced into the equation for each dimensional term.

$$
\begin{aligned}
& {\left[\frac{\partial\left(U \cdot u_{o}\right)}{\partial(X \cdot L)}+\frac{\partial\left(V \cdot u_{o}\right)}{\partial(Y \cdot L)}+\frac{\partial\left(W \cdot u_{o}\right)}{\partial(Z \cdot L)}\right]=0} \\
& \frac{u_{o}}{L}\left[\frac{\partial U}{\partial X}+\frac{\partial V}{\partial Y}+\frac{\partial W}{\partial Z}\right]=0
\end{aligned}
$$

This further simplifies to give the non-dimensional form of the continuity equation. 
$\left[\frac{\partial U}{\partial X}+\frac{\partial V}{\partial Y}+\frac{\partial W}{\partial Z}\right]=0$

X-direction Momentum Balance Equation

$\rho_{f} \cdot\left[u \cdot \frac{\partial u}{\partial x}+v \cdot \frac{\partial u}{\partial y}+w \cdot \frac{\partial u}{\partial z}\right]=-\frac{\partial p}{\partial x}+\mu \cdot\left[\frac{\partial^{2} u}{\partial x^{2}}+\frac{\partial^{2} u}{\partial y^{2}}+\frac{\partial^{2} u}{\partial z^{2}}\right]$

Left hand side:

$\rho_{f} \cdot\left[\left(U \cdot u_{o}\right) \cdot \frac{\partial\left(U \cdot u_{o}\right)}{\partial(X \cdot L)}+\left(V \cdot u_{o}\right) \cdot \frac{\partial(U \cdot L)}{\partial(Y \cdot L)}+\left(W \cdot u_{o}\right) \cdot \frac{\partial\left(U \cdot u_{o}\right)}{\partial(Z \cdot L)}\right]$

Right hand side:

$-\frac{\partial\left(\frac{P \cdot \mu \cdot u_{o}}{L}\right)}{\partial(X \cdot L)}+\mu \cdot\left[\frac{\partial^{2}\left(U \cdot u_{o}\right)}{\partial(X \cdot L)^{2}}+\frac{\partial^{2}\left(U \cdot u_{o}\right)}{\partial(Y \cdot L)^{2}}+\frac{\partial^{2}\left(U \cdot u_{o}\right)}{\partial(Z \cdot L)^{2}}\right]$

These equations are then simplified by taking out the common variables on both the left hand side and right hand side:

$\rho_{f} \cdot \frac{u_{o}^{2}}{L}\left[U \cdot \frac{\partial U}{\partial X}+V \cdot \frac{\partial U}{\partial Y}+W \cdot \frac{\partial U}{\partial Z}\right]=-\frac{\mu \cdot u_{o}}{L^{2}} \cdot \frac{\partial P}{\partial X}+\frac{\mu \cdot u_{o}}{L^{2}} \cdot\left[\frac{\partial^{2} U}{\partial X^{2}}+\frac{\partial^{2} U}{\partial Y^{2}}+\frac{\partial^{2} U}{\partial Z^{2}}\right]$

Multiplying the above equation by a factor of $\frac{L^{2}}{\mu \cdot u_{o}}$ to further simplify the equation gives: 
$\operatorname{Re} \cdot\left[U \cdot \frac{\partial U}{\partial X}+V \cdot \frac{\partial U}{\partial Y}+W \cdot \frac{\partial U}{\partial Z}\right]=-\frac{\partial P}{\partial X}+\left[\frac{\partial^{2} U}{\partial X^{2}}+\frac{\partial^{2} U}{\partial Y^{2}}+\frac{\partial^{2} U}{\partial Z^{2}}\right]$

Where:

$\operatorname{Re}=\frac{\rho_{f} \cdot u_{o} \cdot L}{\mu}$

Y-direction Momentum Balance Equation

$\rho_{f} \cdot\left[u \cdot \frac{\partial v}{\partial x}+v \cdot \frac{\partial v}{\partial y}+w \cdot \frac{\partial v}{\partial z}\right]=-\frac{\partial p}{\partial y}+\mu \cdot\left[\frac{\partial^{2} v}{\partial x^{2}}+\frac{\partial^{2} v}{\partial y^{2}}+\frac{\partial^{2} v}{\partial z^{2}}\right]-\rho \cdot \beta_{T} \cdot\left(T-T_{C}\right) \cdot g$

Substitute in the non-dimensional terms and take out common variables to get:

$\rho_{f} \cdot \frac{u_{o}^{2}}{L} \cdot\left[U \cdot \frac{\partial V}{\partial X}+V \cdot \frac{\partial V}{\partial Y}+W \frac{\partial V}{\partial Z}\right]=-\frac{\mu \cdot u_{o}}{L^{2}} \cdot \frac{\partial P}{\partial Y}+\frac{\mu \cdot u_{o}}{L^{2}}\left[\frac{\partial^{2} V}{\partial X^{2}}+\frac{\partial^{2} V}{\partial Y^{2}}+\frac{\partial^{2} V}{\partial Z^{2}}\right]-\rho \cdot \beta_{T} \cdot\left(T-T_{C}\right) \cdot \ell$

Multiply the above equation by the factor of $\frac{L^{2}}{\mu \cdot u_{o}}$. It should be noted that $\left(T-T_{C}\right)=\left(T_{H}-T_{C}\right) \cdot \theta$

$\operatorname{Re} \cdot\left[U \cdot \frac{\partial V}{\partial X}+V \cdot \frac{\partial V}{\partial Y}+W \frac{\partial V}{\partial Z}\right]=-\frac{\partial P}{\partial Y}+\left[\frac{\partial^{2} V}{\partial X^{2}}+\frac{\partial^{2} V}{\partial Y^{2}}+\frac{\partial^{2} V}{\partial Z^{2}}\right]-\frac{L^{2}}{\mu \cdot u_{o}} \cdot \rho \cdot \beta_{T} \cdot\left(T_{H}-T_{C}\right) \cdot g \cdot \theta$

Simplify the * term:

$*=\frac{L^{2} \cdot \rho \cdot \beta_{T} \cdot g \cdot\left(T_{H}-T_{C}\right)}{\mu \cdot u_{o}}$

Add and subtract $T_{I}$ in the $\left(T_{H^{-}} T_{C}\right)$ term to get: 
$*=\frac{L^{2} \cdot \rho \cdot \beta_{T} \cdot g \cdot\left[\left(T_{H}-T_{I}\right)+\left(T_{I}-T_{C}\right)\right]}{\mu \cdot u_{o}}$

Substitute in $v=\frac{\mu}{\rho}$ and separate the two terms:

$$
*=\frac{L^{2} \cdot g \cdot \beta_{T} \cdot\left(T_{H}-T_{1}\right)}{v \cdot u_{o}}+\frac{L^{2} \cdot g \cdot \beta_{T} \cdot\left(T_{1}-T_{C}\right)}{v \cdot u_{o}}
$$

The liquid Rayleigh number and porous Rayleigh number for the bottom heating case are defined as:

$$
R a_{L}=\frac{g \cdot \beta_{T} \cdot d_{1}^{3} \cdot\left(T_{I}-T_{C}\right)}{\alpha \cdot v}, \quad R a_{P}=\frac{g \cdot \beta_{T} \cdot d_{2} \cdot \kappa \cdot\left(T_{H}-T_{I}\right)}{\alpha \cdot v}
$$

Substitute those into the equation to get:

$$
*=\frac{\alpha \cdot R a_{P} \cdot L^{2}}{d_{2} \cdot \kappa \cdot u_{0}}+\frac{\alpha \cdot R a_{L} \cdot L^{2}}{u_{0} \cdot d_{1}^{3}}
$$

From the Reynolds number, substitute in $u_{o}=\frac{\operatorname{Re} \cdot \mu}{\rho_{f} \cdot L}$, the Prandtl number, $\operatorname{Pr}=\frac{\mu}{\alpha \cdot \rho}$, and the Darcy number, $D a=\frac{\kappa}{L^{2}}$.

$*=\frac{R a_{P} \cdot L}{d_{2} \cdot D a \cdot \operatorname{Re} \cdot \operatorname{Pr}}+\frac{R a_{L} \cdot L^{3}}{\operatorname{Re} \cdot \operatorname{Pr} \cdot d_{1}^{3}}$

Note that $L=d_{1}+d_{2}$. Hence, 


$$
*=\frac{1}{\operatorname{Re} \cdot \operatorname{Pr}} \cdot\left[\frac{R a_{P}}{D a} \cdot\left(1+\frac{d_{1}}{d_{2}}\right)+R a_{L} \cdot\left(1+\frac{d_{2}}{d_{1}}\right)^{3}\right]
$$

A definition of the Reynolds number for FIDAP 8.7.0 can be formulated as follows if the characteristic velocity is taken as $u_{o}=\sqrt{g \cdot \beta_{T} \cdot \Delta T \cdot L}$, with the $\Delta T=T_{H}-T_{C}$.

$\operatorname{Re}=\frac{u_{o} \cdot L}{v}$

$\operatorname{Re}=\frac{\sqrt{g \cdot \beta_{T} \cdot \Delta T \cdot L} \cdot L}{v}$

$\operatorname{Re}^{2}=\frac{g \cdot \beta_{T} \cdot \Delta T \cdot L^{3}}{v^{2}}$

Add and subtract $T_{I}$ in the $\Delta T$ term, and separate the variables.

$$
\operatorname{Re}^{2}=\frac{g \cdot \beta_{T} \cdot L^{3}}{v^{2}} \cdot\left(T_{H}-T_{I}\right)+\frac{g \cdot \beta_{T} \cdot L^{3}}{v^{2}} \cdot\left(T_{I}-T_{C}\right)
$$

Substitute in the liquid and porous Rayleigh numbers, as well as the Darcy and Prandtl numbers. Also note that $L=d_{1}+d_{2}$. The Reynolds number can now be defined as:

$$
\operatorname{Re}=\sqrt{\frac{1}{\operatorname{Pr}} \cdot\left[\frac{R a_{P}}{D a} \cdot\left(1+\frac{d_{1}}{d_{2}}\right)+R a_{L} \cdot\left(1+\frac{d_{2}}{d_{1}}\right)^{3}\right]}
$$

Now introduce the defined Reynolds number into the simplified * term. 


$$
*=\frac{\frac{R a_{P}}{D a} \cdot\left(1+\frac{d_{1}}{d_{2}}\right)+R a_{L} \cdot\left(1+\frac{d_{2}}{d_{1}}\right)^{3}}{\operatorname{Pr} \cdot \sqrt{\frac{1}{\operatorname{Pr}} \cdot\left[\frac{R a_{P}}{D a} \cdot\left(1+\frac{d_{1}}{d_{2}}\right)+R a_{L} \cdot\left(1+\frac{d_{2}}{d_{1}}\right)^{3}\right]}}
$$

Next, the denominator is rationalized to reveal the final simplified * term:

$$
*=\sqrt{\frac{1}{\operatorname{Pr}} \cdot\left[\frac{R a_{P}}{D a} \cdot\left(1+\frac{d_{1}}{d_{2}}\right)+R a_{L} \cdot\left(1+\frac{d_{2}}{d_{1}}\right)^{3}\right]}
$$

Therefore, the final form of the non-dimensional equation in the $y$-direction can be expressed as:

$\operatorname{Re} \cdot\left[U \cdot \frac{\partial V}{\partial X}+V \cdot \frac{\partial V}{\partial Y}+W \frac{\partial V}{\partial Z}\right]=-\frac{\partial P}{\partial Y}+\left[\frac{\partial^{2} V}{\partial X^{2}}+\frac{\partial^{2} V}{\partial Y^{2}}+\frac{\partial^{2} V}{\partial Z^{2}}\right]-\operatorname{Re} \cdot \theta$

\section{Energy Equation}

$$
\left(\rho \cdot C_{p}\right)_{f} \cdot\left[u \cdot \frac{\partial T}{\partial x}+v \cdot \frac{\partial T}{\partial y}+w \frac{\partial T}{\partial z}\right]=k_{f} \cdot\left[\frac{\partial^{2} T}{\partial x^{2}}+\frac{\partial^{2} T}{\partial y^{2}}+\frac{\partial^{2} T}{\partial z^{2}}\right]
$$

Substitute in the non-dimensional parameters to obtain:

Left hand side:

$$
\left(\rho \cdot C_{p}\right)_{f} \cdot\left[\left(U \cdot u_{o}\right) \cdot \frac{\partial \theta}{\partial(X \cdot L)}+\left(V \cdot u_{o}\right) \cdot \frac{\partial \theta}{\partial(Y \cdot L)}+\left(W \cdot u_{o}\right) \cdot \frac{\partial \theta}{\partial(Z \cdot L)}\right]
$$

Right hand side: 


$$
k_{f} \cdot\left[\frac{\partial^{2} \theta}{\partial(X \cdot L)^{2}}+\frac{\partial^{2} \theta}{\partial(Y \cdot L)^{2}}+\frac{\partial^{2} \theta}{\partial(Z \cdot L)^{2}}\right]
$$

Extract the common variables from the left hand side and the right hand side.

$$
\left(\rho \cdot C_{p}\right)_{f} \cdot \frac{u_{o} \cdot L}{k_{f}} \cdot\left[\frac{\partial \theta}{\partial \tau}+U \cdot \frac{\partial \theta}{\partial X}+V \cdot \frac{\partial \theta}{\partial Y}\right]=\left[\frac{\partial^{2} \theta}{\partial X^{2}}+\frac{\partial^{2} \theta}{\partial Y^{2}}\right]
$$

Substitute in the Reynolds number, $\operatorname{Re}=\frac{\rho_{f} \cdot u_{o} \cdot L}{\mu}$, and the Prandtl number, $\operatorname{Pr}=\frac{\mu \cdot\left(C_{P}\right)_{f}}{k_{f}}$, to get the non-dimensional form of the energy equation.

$\operatorname{Re} \cdot \operatorname{Pr} \cdot\left[U \cdot \frac{\partial \theta}{\partial X}+V \cdot \frac{\partial \theta}{\partial Y}+W \cdot \frac{\partial \theta}{\partial Z}\right]=\left[\frac{\partial^{2} \theta}{\partial X^{2}}+\frac{\partial^{2} \theta}{\partial Y^{2}}+\frac{\partial^{2} \theta}{\partial Z^{2}}\right]$

\section{Porous Layer}

\section{Continuity Equation}

For the derivation of the non-dimensional continuity equation, refer to the liquid layer section above.

X-direction Momentum Balance Equation

$$
\frac{\mu \cdot u}{\kappa}=-\frac{\partial p}{\partial x}+\mu_{e} \cdot\left[\frac{\partial^{2} u}{\partial x^{2}}+\frac{\partial^{2} u}{\partial y^{2}}+\frac{\partial^{2} u}{\partial z^{2}}\right]
$$

Introduce the dimensionless parameters and factor out the common variables: 


$$
\frac{\mu \cdot\left(U \cdot u_{o}\right)}{\kappa}=-\frac{\mu \cdot u_{o}}{L^{2}} \cdot \frac{\partial P}{\partial X}+\frac{\mu \cdot u_{o}}{L^{2}} \cdot\left[\frac{\partial^{2} U}{\partial X^{2}}+\frac{\partial^{2} U}{\partial Y^{2}}+\frac{\partial^{2} U}{\partial Z^{2}}\right] \nabla
$$

Multiply the equation by the factor of $\frac{L^{2}}{\mu \cdot u_{o}}$ to further simplify the equation.

$$
\frac{L^{2}}{\kappa} \cdot U=-\frac{\partial P}{\partial X}+\left[\frac{\partial^{2} U}{\partial X^{2}}+\frac{\partial^{2} U}{\partial Y^{2}}+\frac{\partial^{2} U}{\partial Z^{2}}\right]
$$

Substitute in the Darcy number $D a=\frac{\kappa}{L^{2}}$ to get the final non-dimensional form of the equation:

$$
\left[\frac{1}{D a}\right] \cdot U=-\frac{\partial P}{\partial X}+\left[\frac{\partial^{2} U}{\partial X^{2}}+\frac{\partial^{2} U}{\partial Y^{2}}+\frac{\partial^{2} U}{\partial Z^{2}}\right]
$$

\section{Energy Equation}

$$
\left(\rho \cdot C_{P}\right)_{f} \cdot\left[u \cdot \frac{\partial T}{\partial x}+v \cdot \frac{\partial T}{\partial y}+w \cdot \frac{\partial T}{\partial z}\right]=k_{e} \cdot\left[\frac{\partial^{2} T}{\partial x^{2}}+\frac{\partial^{2} T}{\partial y^{2}}+\frac{\partial^{2} T}{\partial z^{2}}\right]
$$

Introduce the dimensionless variables into the above equation to receive the following:

Left hand side:

$$
\left(\rho \cdot C_{p}\right)_{f} \cdot\left[\left(U \cdot u_{o}\right) \cdot \frac{\partial \theta}{\partial(X \cdot L)}+\left(V \cdot u_{o}\right) \cdot \frac{\partial \theta}{\partial(Y \cdot L)}+\left(W \cdot u_{o}\right) \cdot \frac{\partial \theta}{\partial(Z \cdot L)}\right]
$$

Right hand side: 
$G \cdot\left[\frac{\partial^{2} \theta}{\partial(X \cdot L)^{2}}+\frac{\partial^{2} \theta}{\partial(Y \cdot L)^{2}}+\frac{\partial^{2} \theta}{\partial(Z \cdot L)^{2}}\right]$

Extract the common variables from the left hand side and the right hand side.

Substitute in the Reynolds number, $\operatorname{Re}=\frac{\rho_{f} \cdot u_{o} \cdot L}{\mu}$, and the Prandtl number, $\operatorname{Pr}=\frac{\mu \cdot\left(C_{P}\right)_{f}}{k_{f}}$, to get the non-dimensional form of the energy equation.

$\operatorname{Re} \cdot \operatorname{Pr} \cdot\left[U \cdot \frac{\partial \theta}{\partial X}+V \cdot \frac{\partial \theta}{\partial Y}+W \cdot \frac{\partial \theta}{\partial Z}\right]=G \cdot\left[\frac{\partial^{2} \theta}{\partial X^{2}}+\frac{\partial^{2} \theta}{\partial Y^{2}}+\frac{\partial^{2} \theta}{\partial Z^{2}}\right]$

Where the non-dimensional overall thermal conductivity is:

$G=\phi+(1-\phi) \cdot \frac{k_{s}}{k_{f}}$

\section{B.2 Lateral Heating}

The derivations of the continuity, $X$ and $Z$ direction momentum balance, and energy equations are similar to those for the bottom heating condition and given above.

Y-direction Momentum Balance Equation

$\rho_{f} \cdot\left[u \cdot \frac{\partial v}{\partial x}+v \cdot \frac{\partial v}{\partial y}+w \cdot \frac{\partial v}{\partial z}\right]=-\frac{\partial p}{\partial y}+\mu \cdot\left[\frac{\partial^{2} v}{\partial x^{2}}+\frac{\partial^{2} v}{\partial y^{2}}+\frac{\partial^{2} v}{\partial z^{2}}\right]-\rho \cdot \beta_{T} \cdot\left(T-T_{C}\right) \cdot g$

Substitute in the non-dimensional terms and take out common variables: 
$\rho_{f} \cdot \frac{u_{o}^{2}}{L} \cdot\left[U \cdot \frac{\partial V}{\partial X}+V \cdot \frac{\partial V}{\partial Y}+W \frac{\partial V}{\partial Z}\right]=-\frac{\mu \cdot u_{o}}{L^{2}} \cdot \frac{\partial P}{\partial Y}+\frac{\mu \cdot u_{o}}{L^{2}}\left[\frac{\partial^{2} V}{\partial X^{2}}+\frac{\partial^{2} V}{\partial Y^{2}}+\frac{\partial^{2} V}{\partial Z^{2}}\right]-\rho \cdot \beta_{T} \cdot\left(T-T_{c}\right) \cdot g$

Multiply the above equation by $\frac{L^{2}}{\mu \cdot u_{o}}$, and note that $\left(T-T_{C}\right)=\left(T_{H}-T_{C}\right) \cdot \theta$.

$\operatorname{Re} \cdot\left[U \cdot \frac{\partial V}{\partial X}+V \cdot \frac{\partial V}{\partial Y}+W \frac{\partial V}{\partial Z}\right]=-\frac{\partial P}{\partial Y}+\left[\frac{\partial^{2} V}{\partial X^{2}}+\frac{\partial^{2} V}{\partial Y^{2}}+\frac{\partial^{2} V}{\partial Z^{2}}\right]-\frac{L^{2}}{\mu \cdot u_{o}} \cdot \rho \cdot \beta_{T} \cdot\left(T_{H}-T_{C}\right) \cdot g \cdot \theta$

Simplify the ${ }^{* *}$ term:

$* *=\frac{L^{2} \cdot \rho \cdot \beta_{T} \cdot g \cdot\left(T_{H}-T_{C}\right)}{\mu \cdot u_{o}}$

For lateral heating case, the liquid Rayleigh and porous Rayleigh numbers are defined as follows:

$$
R a_{L}=\frac{g \cdot \beta_{T} \cdot d_{1}^{3} \cdot\left(T_{H}-T_{C}\right)}{\alpha \cdot v}, \quad R a_{P}=\frac{g \cdot \beta_{T} \cdot d_{2} \cdot \kappa \cdot\left(T_{H}-T_{C}\right)}{\alpha \cdot v}
$$

They are also related by:

$$
R a_{P}=R a_{L} \cdot D a \cdot \frac{d_{2} \cdot\left(d_{1}+d_{2}\right)^{2}}{d_{1}^{3}}
$$

Substitute the liquid Rayleigh number into the ** term:

$* *=\frac{L^{2} \cdot \rho \cdot R a_{L} \cdot \alpha \cdot v}{\mu \cdot u_{0} \cdot d_{1}^{3}}$ 
Substitute in the Prandtl number, $\operatorname{Pr}=\frac{\mu \cdot\left(C_{P}\right)_{f}}{k_{f}}$, the thermal diffusivity of the fluid, $\alpha=\frac{k_{f}}{\rho_{f} \cdot\left(C_{P}\right)_{f}}$, and the Reynolds number, $\operatorname{Re}=\frac{\rho_{f} \cdot u_{o} \cdot L}{\mu}$.

$* *=\frac{R a_{L} \cdot L^{3}}{\operatorname{Pr} \cdot \operatorname{Re} \cdot d_{1}^{3}}$

Noting that $L=d_{1}+d_{2}$, the equation becomes:

$* *=\frac{1}{\operatorname{Re}} \cdot \frac{\operatorname{Ra}}{\operatorname{Pr}} \cdot\left(1+\frac{d_{2}}{d_{1}}\right)^{3}$

By assuming that $u_{o}=\sqrt{g \cdot \beta_{r} \cdot \Delta T \cdot L}$, the Reynolds number can now be defined as:

$\operatorname{Re}=\sqrt{\frac{\operatorname{Ra} a_{L}}{\operatorname{Pr}} \cdot\left(1+\frac{d_{2}}{d_{1}}\right)^{3}}$

The final form of the non-dimensional equation in the $y$-direction can be expressed as:

$\operatorname{Re} \cdot\left[U \cdot \frac{\partial V}{\partial X}+V \cdot \frac{\partial V}{\partial Y}+W \frac{\partial V}{\partial Z}\right]=-\frac{\partial P}{\partial Y}+\left[\frac{\partial^{2} V}{\partial X^{2}}+\frac{\partial^{2} V}{\partial Y^{2}}+\frac{\partial^{2} V}{\partial Z^{2}}\right]-\operatorname{Re} \cdot \theta$ 


\section{APPENDIX B}

\section{Input Files}

\section{C.1 Marangoni Convection Lateral Heating}

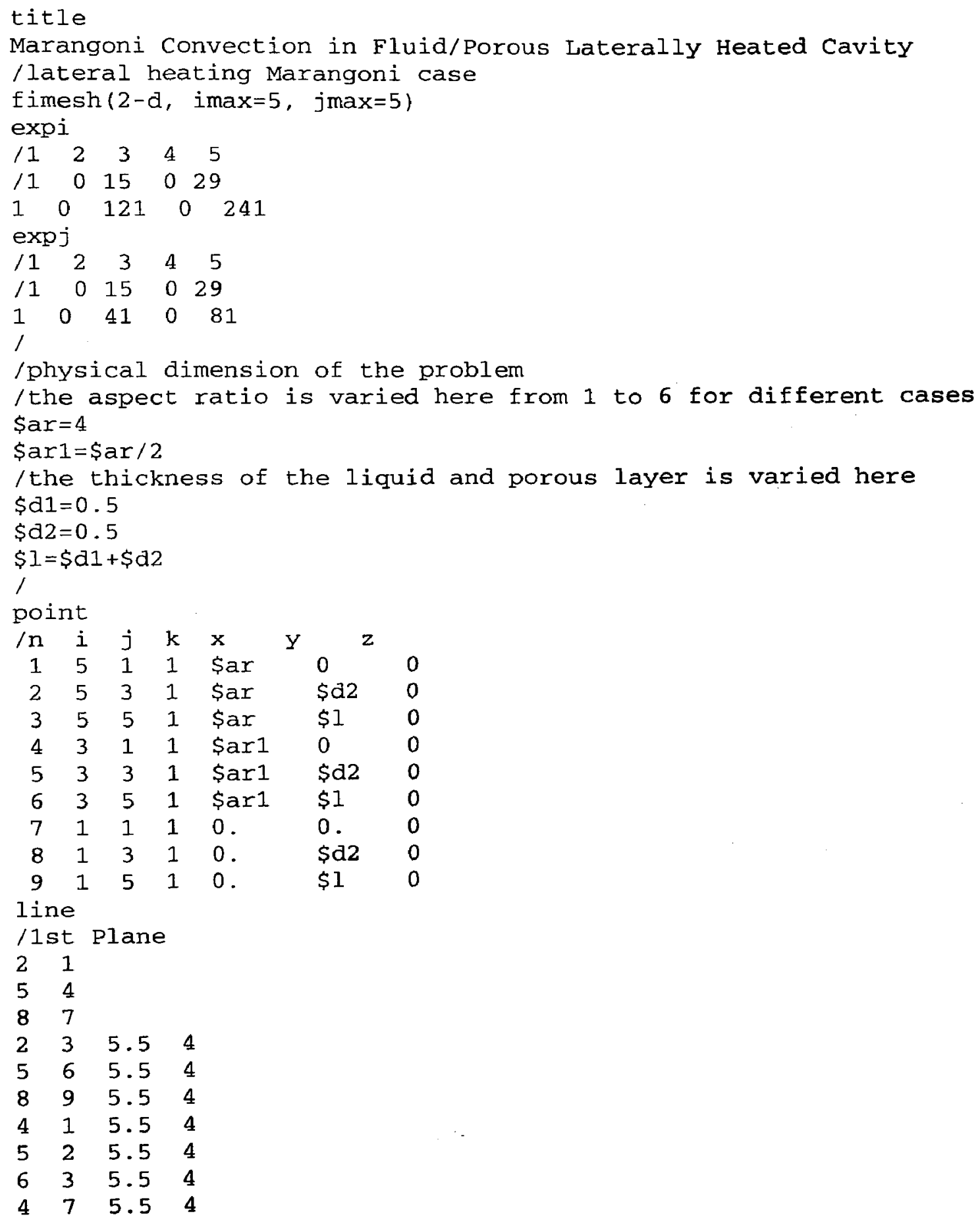




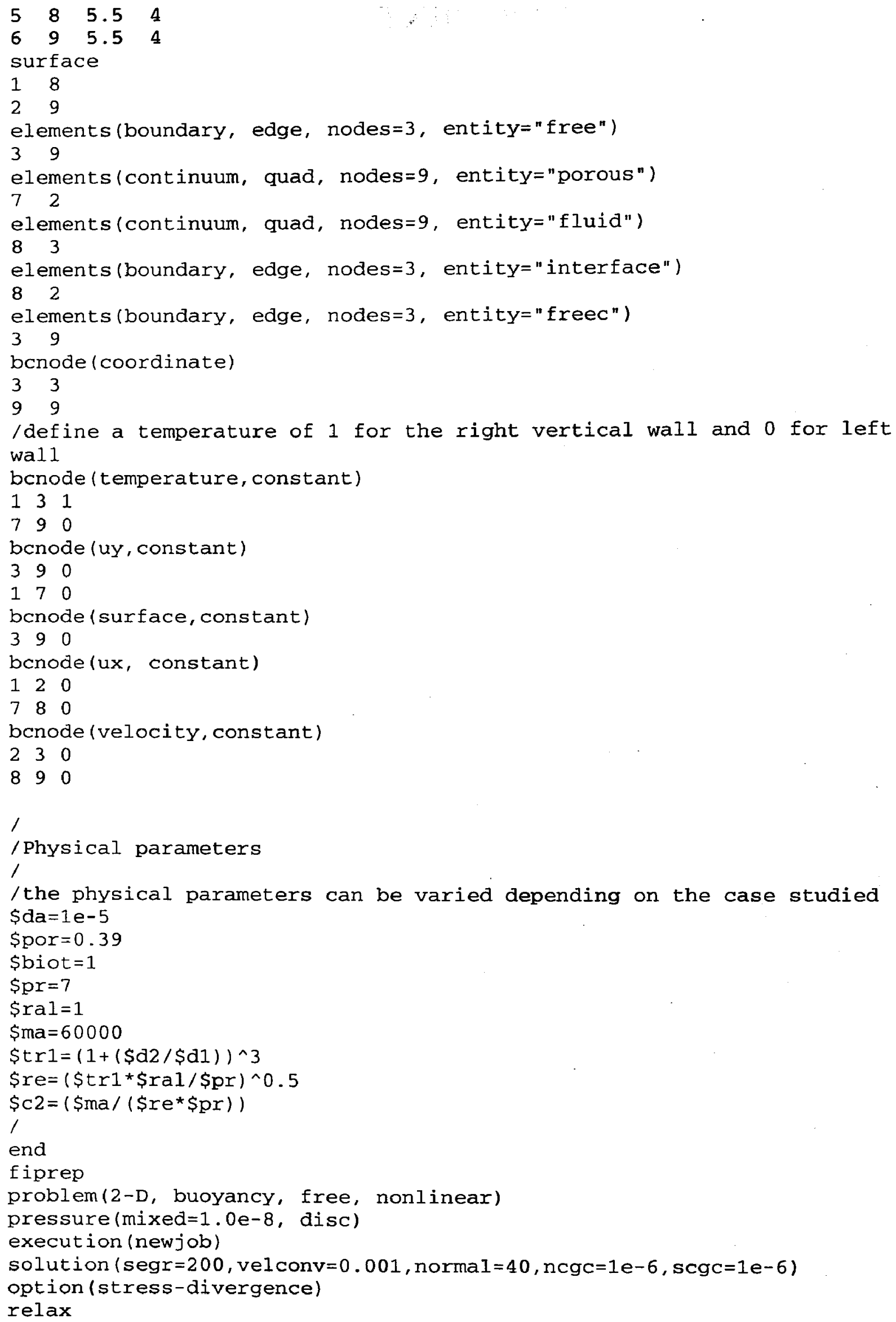




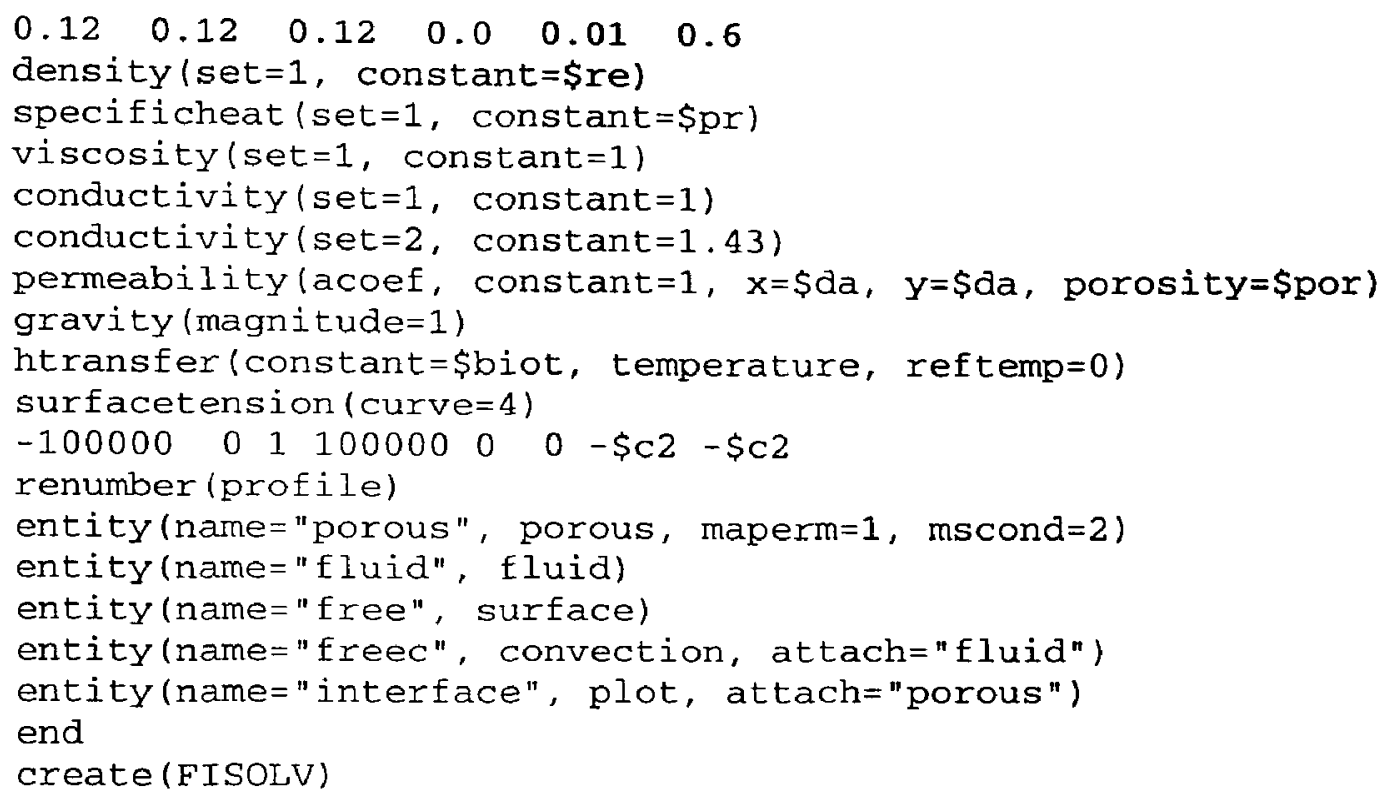

\section{C.2 Evaporation Lateral Heating}

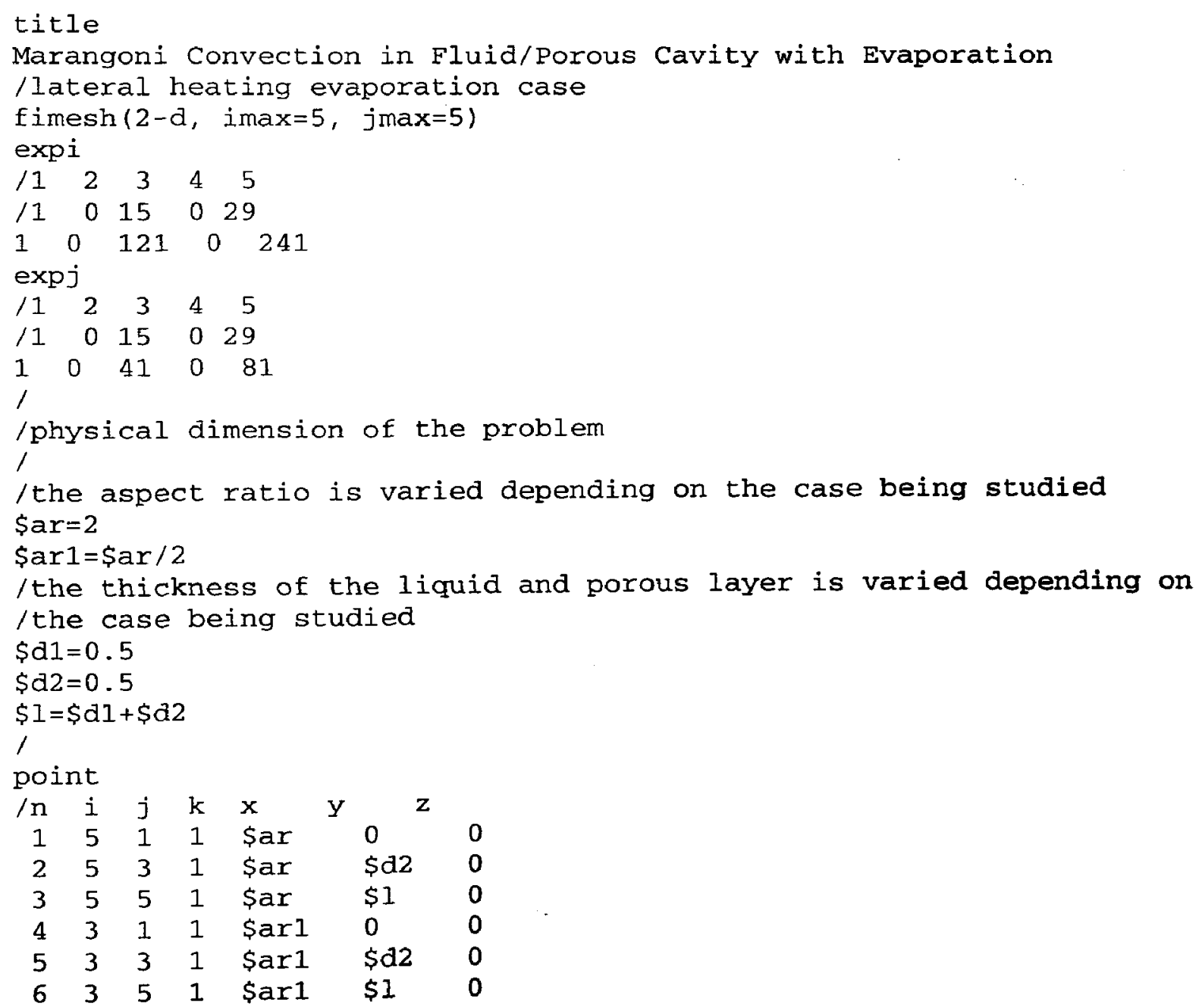




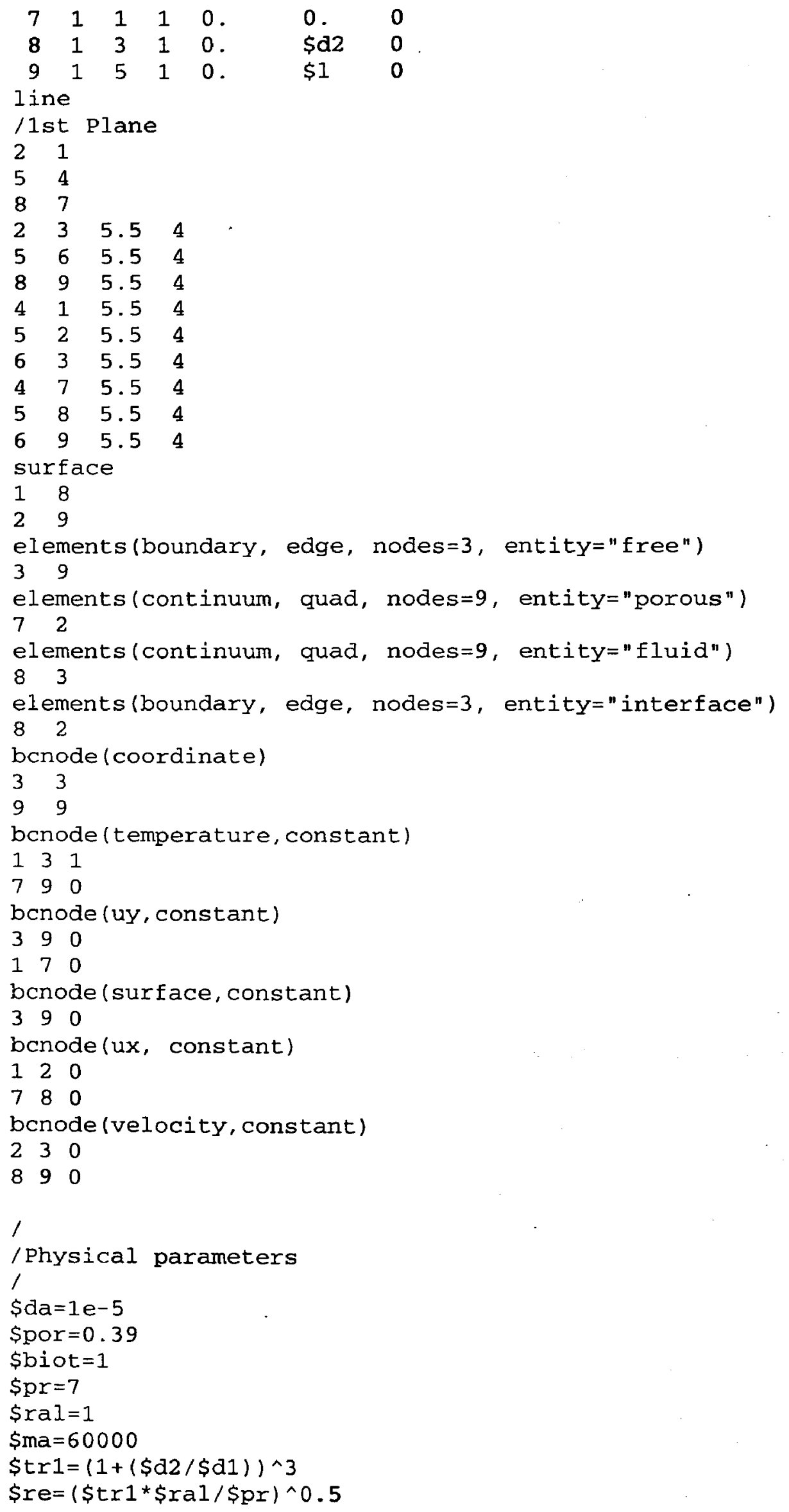




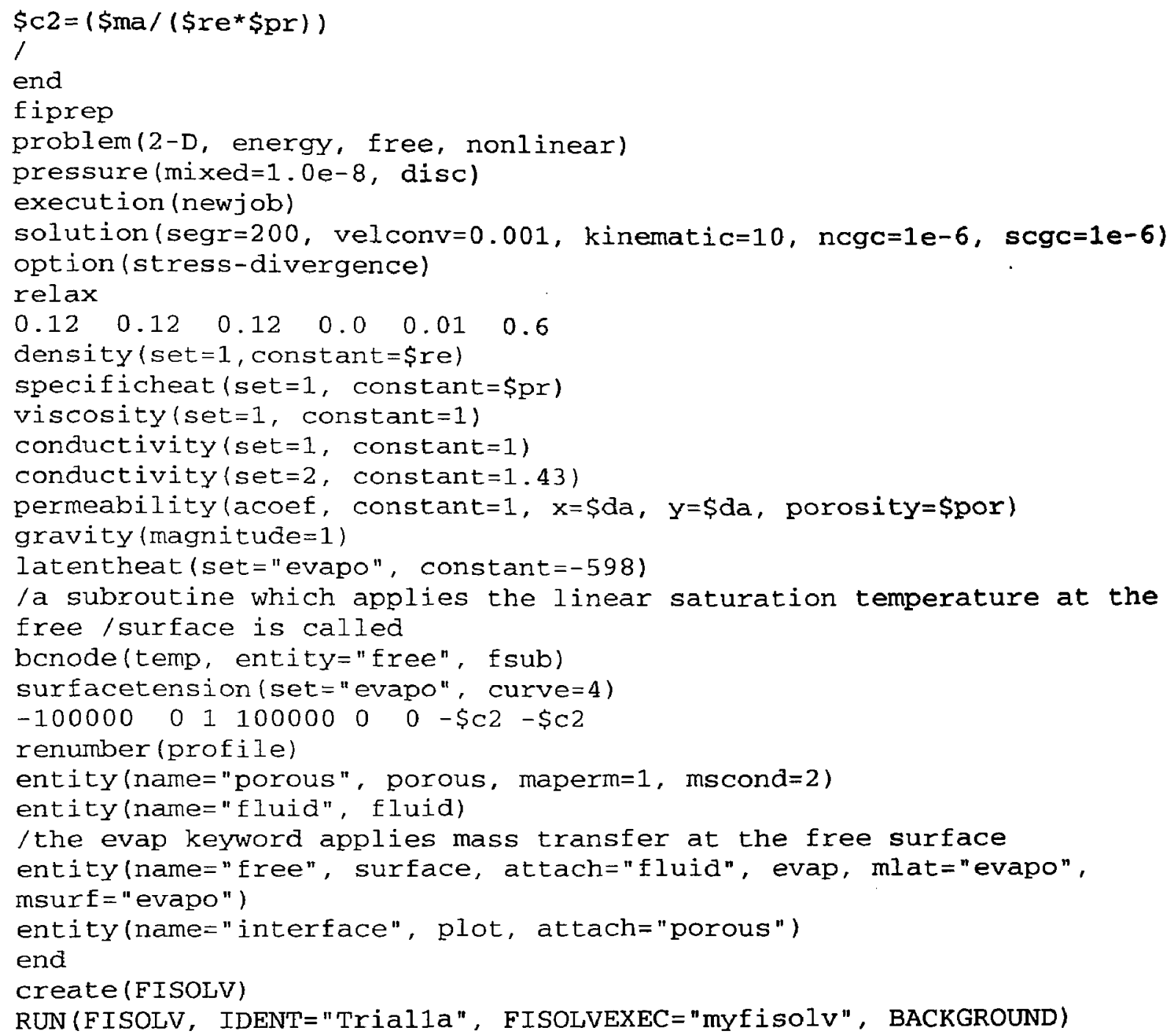





\section{REFERENCES}

[1] D. A. Nield, A. Bejan, Convection in Porous Media, Springer-Verlag: New York, 1992.

[2] V. P. Carey, Liquid-Vapor Phase-Change Phenomena: An Introduction to the Thermophysics of Vaporization and Condensation Processes in Heat Transfer Equipment, Taylor \& Francis: Bristol PA, 1992.

[3] G. S. Beavers, D. D. Joseph, "Boundary conditions at a naturally permeable wall," J. Fluid Mechanics 30 (1967) 197-207.

[4] D. A. Nield, "Onset of convection in a fluid layer overlying a layer of a porous medium," J. Fluid Mechanics 81 (1977) 513-522.

[5] F. Chen, C. F. Chen, "Onset of finger convection in a horizontal porous layer underlying a fluid layer," J. Heat Transfer 110 (1988) 403-409.

[6] F. Chen, C. F. Chen, "Experimental investigation of convective stability in a superposed fluid and porous layer when heated from below," J. Fluid Mechanics 207 (1989) 311-321.

[7] S. J. Kim, C. Y. Choi, "Convective heat transfer in porous and overlying fluid layers heated from below," Int. J. Heat Mass Transfer 39 (1996) 319-329.

[8] T. Desaive, G. Lebon, M. Hennenberg, "Coupled capillary and gravity-driven instability in a liquid film overlying a porous layer," Physical Review E 64 (2001).

[9] M. Z. Saghir, P. Comi, M. Mehrvar, "Effects of interaction between Rayleigh and 
Marangoni convection in superposed fluid and porous layers," Int. J. Thermal Sciences 413 (2002) 207-215.

[10] D. Villers, J. K. Platten, "Coupled buoyancy and Marangoni convection in acetone: experiments and comparison with numerical simulations," J. Fluid Mechanics 234 (1992) 487-510.

[11] M. Z. Saghir, P. Mahendran, M. Hennenberg, "Marangoni and gravity driven convection in a liquid layer overlying a porous layer: Lateral and bottom heating conditions," Energy Sources 27 (2005) 151 171.

[12] P. C. Dauby, G. Lebon, "Benard-Marangoni instability in rigid rectangular containers," J. Fluid Mechanics 329 (1996) 25-64.

[13] M. F. Schatz, S. J. VanHook, W. D. McCormick, J. B. Swift, H. L. Swinney "Onset of Surface-Tension-Driven Benard Convection," Physical Review E 75 (1995) 1938-1941.

[14] P. M. Parmentier, V. C. Regnier, G. Lebon, J. C. Legros, "Nonlinear analysis of coupled gravitational and capillary thermoconvection in thin fluid layers," Physical Review E 54 (1996) 411-423.

[15] V. Regnier, P. C. Dauby, P. Parmentier, G. Legon, "Square cells in gravitational and capillary thermoconvection," Physical Review E 55 (1996) 6860-6865.

[16] D. E. Haas, D. P. Birnie, "Evaluation of thermocapillary driving forces in the development of striations during the spin coating process," J. Material Science 37 (2002) 2109-2116.

[17] F. Mashayek, "Dynamics of evaporating drops. Part I: formulation and evaporation model," Int. J. Heat Mass Transfer 44 (2001) 1517-1526. 
[18] V. M. Ha, C. L. Lai, "Onset of Marangoni instability of a two-component evaporation droplet," Int. J. Heat Mass Transfer 45 (2002) 5143-5158.

[19] A. T. Shih, C. M. Megaridis, "Thermocapillary flow effects on convective droplet evaporation," Int. J. Heat Mass Transfer 39 (1996) 247-257.

[20] G. Fang, C. A. Ward, "Temperature measured close to the interface of an evaporating liquid," Physical Review E 59 (1999) 417-428.

[21] C. A. Ward, D. Stanga, "Interfacial conditions during evaporation or condensation of water," Physical Review E 64 (2001).

[22] C. A. Ward, F. Duan, D. Stanga, Z. Saghir, "Characteristics of Marangoni-Benard Convection During Water Evaporation," Microgravity Transport Processes in Fluid, Thermal, Materials, and Biological Sciences II, Sept. 30 - Oct. 5, 2001, Banff, Canada.

[23] FIDAP USER MANUAL, Volume 8.7.0, 1999

[24] F. M. White, Fluid Mechanics $4^{\text {th }}$ Edition, WCM/McGraw-Hill: Toronto, 1999. 